Laser-Induced Forward Transfer of Pure Metals

// Towards 3D Printing 


\section{LASER-INDUCED FORWARD TRANSFER OF PURE METALS}

TOWARDS 3D PRINTING

Ralph Pohl 


\section{Composition of the graduation committee:}

Chairman and secretary:

Prof. dr. G.P.M.R. Dewulf University of Twente, The Netherlands

Promoter and assistant-promoter:

Prof. dr. ir. Just L. Herder University of Twente, The Netherlands

Dr. ir. G.R.B.E. Römer University of Twente, The Netherlands

Members:

Prof. dr. Philippe Delaporte

Aix-Marseille University, France

Prof. dr. Quan Zhou

Prof. dr. Detlef Lohse

Aalto University, Finland

Prof. dr. ir. Remko Akkerman

Dr. ir. Ton Bor

University of Twente, The Netherlands

University of Twente, The Netherlands

University of Twente, The Netherlands

The work described in this thesis was performed at the group of Mechanical Automation of the Faculty of Engineering Technology, Chair of Applied Laser Technology, University of Twente, P.O. Box 217, 7500 AE Enschede, The Netherlands.

This research was carried out in the framework of the European Union Seventh Framework Programme under Grant Agreement No. 260079 (FAB2ASM).

Laser-induced forward transfer of pure metals

Ralph Pohl

PhD Thesis, University of Twente, Enschede, The Netherlands

September 2015

ISBN 978-90-365-3869-5

DOI 10.3990/1.9789036538695

Copyright (C) 2015 by Ralph Pohl, The Netherlands

Printed by Gildeprint. 


\title{
LASER-INDUCED FORWARD TRANSFER OF PURE METALS
}

\author{
TOWARDS 3D PRINTING
}

\section{DISSERTATION}

to obtain

the degree of doctor at the University of Twente, on the authority of the rector magnificus, prof. dr. H. Brinksma, on account of the decision of the graduation committee, to be publicly defended on Wednesday $16^{\text {th }}$ of September 2015 at 14.45

by

\section{Ralph Pohl}

born on $6^{\text {th }}$ of October 1981

in Gronau, Germany 
This thesis has been approved by the promoter

Prof. dr. ir. Just L. Herder

and the co-promoter

Dr. ir. G.R.B.E Römer 


\section{Summary}

Additive manufacturing offers several advantages compared to conventional methods of production, such as an increased freedom of design and a toolless production suited for variable lot sizes. In particular the printing concept has gained momentum for rapid prototyping and manufacturing, since it allows for unprecedented freedom in the design of novel products, and is not limited by the drawback of powder bed fusion methods, such as selective laser melting. However, nozzle based printing of metals remains challenging, since the melting temperature of most metals is similar to components within the printing nozzle. Therefore, metal printing has been limited to low-melting point metals and metal containing inks, which are generally not optimized for other material properties (e.g. strength, conductivity, and corrosion rate) and cost.

Laser-induced forward transfer (LIFT), is a direct-write method allowing for direct deposition of a wide range of materials including metals like aluminum, chromium, copper, gold, nickel and tungsten. In LIFT of metals, the energy of the laser pulse is absorbed by the metal, resulting in the generation of a thermal stress wave or evaporation of a part of the metal film, which subsequently leads to the ejection of a metal micro-droplet. Subsequently the droplet is deposited on the receiving substrate, on which the product is to be printed.

However, the exact ejection mechanisms of these droplets are still under debate. Therefore, this thesis provides a detailed study on the ejection mechanisms during picosecond and nanosecond LIFT of copper and gold. High-speed imaging experiments were performed in order to visualize fluence dependent ejection dynamics and hence to identify and characterize ejection regimes during LIFT. To interpret those ejection regimes, the physical conditions in the metal film were assessed. To that end, the response of the material to the absorbed laser pulse was computed using a numerical two-temperature model. From that, two driving mechanisms, namely laser-induced stress relaxation and the vaporization of the metal film were studied and discussed. It was found that the generated stress distribution is key to the interpretation of the observed ejection dynamics and to the 
explanation of the ejection fluence threshold.

Apart from the ejection dynamics, also the deposition process as well as the production of complex metal parts with dimensions in the micrometer scale were addressed. Hereunto, the presented work provides first studies towards full 3D printing capabilities demonstrated by the manufacturing of high-aspect ratio pillars of copper and gold. In addition to the printing of liquid phase metal droplets, also the transfer of thin metal films in solid phase was studied. To this end, an advanced LIFT setup was developed and successfully employed to demonstrate the solid phase transfer of thin films. 


\section{Publications}

\section{Journal articles}

- Ralph Pohl, Claas Willem Visser, Gert-Willem Römer, Detlef Lohse, Chao Sun, and Bert Huis in 't Veld. Ejection Regimes in Picosecond Laser-Induced Forward Transfer of Metals. Physical Review Applied, 3(2):024001, 2015.

- R. Pohl, C.W. Visser, G.R.B.E. Römer, C. Sun, A.J. Huis in 't Veld, and D. Lohse. Imaging of the ejection process of nanosecond laser-induced forward transfer of gold. Journal of Laser Micro/Nanoengineering, 10(2):154-157, 2015.

- R. Pohl, M. Jansink, G.R.B.E. Römer, A.J. Huis in 't Veld. Solid-phase laserinduced forward transfer of variable shapes using a liquid crystal spatial light modulator. Applied Physics A, 120:427-434, 2015.

- Claas Willem Visser, Ralph Pohl, Chao Sun, Bert Huis in 't Veld, Gert-Willem Römer, and Detlef Lohse. Towards 3D Printing of Pure Metals by Laser-Induced Forward Transfer. Advanced Materials, 27:4087-4092, 2015.

- R. Pohl, C.W. Visser, G.R.B.E. Römer, C. Sun, and D. Lohse. Characterization of the cap ejection regime in laser-induced forward transfer of copper. Submitted for publication, 2015.

\section{Conference articles}

- R. Pohl, G.R.B.E. Römer, M.B. Hoppenbrouwers, A.J. Huis in 't Veld. Characterization of Metal Sprays Created by a Picosecond Laser-Induced Forward Transfer (LIFT) Process. Physics Procedia - Laser Assisted Net shape Engineering 7 (LANE 2012), 39:409-415, 2012.

- R. Pohl, C.W. Visser, G.R.B.E. Römer, C. Sun, A.J. Huis in 't Veld, D. Lohse. Droplet ejection in laser-induced forward transfer: mechanism for 
contamination. Proceedings of LAMP2013 - the 6th International Congress on Laser Advanced Materials Processing, Niigata, Japan, 2013.

- R. Pohl, C.W. Visser, G.R.B.E. Römer, C. Sun, A.J. Huis in 't Veld, D. Lohse. High-resolution imaging of ejection dynamics in laser-induced forward transfer. Proceedings of SPIE 8967, Laser Applications in Microelectronic and Optoelectronic Manufacturing (LAMOM) XIX, 89670X, 2014.

- G.R.B.E. Römer, D. Arnaldo del Cerro, M.M.J. Jorritsma, R. Pohl, B. Chang, V. Liimatainen, Q. Zhou, and A.J. Huis in 't Veld. Laser micromachining of sharp edged receptor sites in polyimide for fluidic driven self-alignment. Proceedings of LPM2012, Washington, DC, USA: Japan Laser Processing Society, 2012.

- G.R.B.E. Römer, D. Arnaldo Del Cerro, R. Pohl, B. Chang, V. Liimatainen, Q. Zhou, A.J. Huis in 't Veld. Picosecond Laser Machining of Metallic and Polymer Substrates for Fluidic Driven Self-Alignment. Physics Procedia - Laser Assisted Net shape Engineering 7 (LANE 2012), 39:628-635, 2012.

- F. Roozeboom, M. Smets, B. Kniknie, M. Hoppenbrouwers, G. Dingemans, W. Keuning, W.M.M. Kessels, R. Pohl, and A.J. Huis in 't Veld. Alternative technology concepts for low-cost and high-speed 2D and 3D interconnect manufacturing. IMAPS-International Microelectronics and Packaging Society, Orlando, Florida, 2013.

- A.J. Huis in 't Veld, M.B. Hoppenbrouwers, M. Giesbers, R. Pohl, G.R.B.E. Römer, C.W. Visser, C. Sun, D. Lohse. Imaging of copper ejection in pico- and nanosecond Laser Induced Forward Transfer. 10th International Conference on Multi-Material Micro Manufacture, San Sebastian, Spain, 2013. 


\section{Contents}

Summary v v

Publications vii

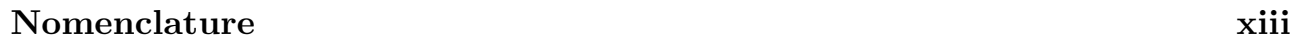

1 Introduction 1

1.1 Additive manufacturing on the micrometer scale . . . . . . . . . . . 1

1.2 Laser-induced forward transfer . . . . . . . . . . . . . . . . . . 2

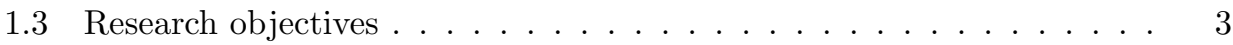

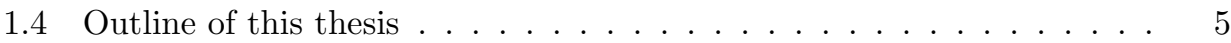

2 State of the art $\quad 7$

2.1 Review on LIFT based processes . . . . . . . . . . . . . . . . . 7

2.1.1 Laser-induced forward transfer

2.1.2 Dynamic release layer LIFT

2.1.3 Matrix-assisted pulsed laser evaporation - direct write

2.1.4 Laser-induced thermal imaging

2.1.5 Long pulsed LIFT

2.1.6 Laser molecular implantation

2.1.7 Summary

2.2 Modeling . . . . . . . . . . . . . . . . . . . . . . . . 11

2.2.1 Review on modeling

2.2.2 Summary

2.3 Time-resolved imaging . . . . . . . . . . . . . . . . . . . . . . . 12

2.3.1 Review on time-resolved imaging

2.3.2 Summary

3 Laser-material interaction and model implementation 
3.1 Interaction of ultra-short laser pulses with metals . . . . . . . . . . . 15

3.1.1 Reflection and absorption in metals

3.1.2 Thermalization

3.2 Thermophysical phenomena . . . . . . . . . . . . . . . . . 19

3.2.1 Stress generation

3.2.2 Phase changes and laser-induced breakdown

3.3 Model implementation . . . . . . . . . . . . . . . . . 22

3.3.1 One-dimensional approach

3.3.2 Two-temperature model

3.3.3 Absorption of the laser pulse

3.3.4 Initial and boundary conditions

3.3.5 Thermophyscial properties

3.3.6 Material parameters

3.3.7 Numerical solver

3.3.8 Validity of the model

4 Experimental methods $\quad 31$

4.1 Introduction . . . . . . . . . . . . . . . . . . . . 31

4.2 Experimental setup . . . . . . . . . . . . . . . . . 31

4.2.1 LIFT Setup

4.2.2 Receiver alignment

4.2.3 Measurement of the gap size

4.2.4 Laser beam delivery

4.2.5 Beam waist

4.3 Materials and sample preparation . . . . . . . . . . . . . . . . 36

4.4 Post-process analysis . . . . . . . . . . . . . . . . . 37

$5 \quad$ Ejection regimes in picosecond LIFT

5.1 Introduction . . . . . . . . . . . . . . . . . . . 39

5.2 Methods . . . . . . . . . . . . . . . . . . . 40

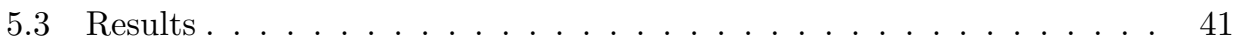

5.3.1 Ejection regime classification

5.3.2 Temperature analysis

5.3.3 Ejection mechanisms

5.4 Discussion . . . . . . . . . . . . . . . . . . . . . . . 48

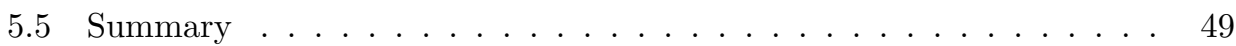

6 Ejection dynamics in picosecond LIFT

6.1 Introduction . . . . . . . . . . . . . . . . . . 51

6.2 Methods . . . . . . . . . . . . . ..... 52

6.3 Results. . . . . . . . . . . . . . . . . . . 52

6.3.1 Cap ejection

6.3.2 Jet ejection

6.3.3 Spray ejection

6.4 Summary .............................. 64 
7 Ejection phenomena in nanosecond LIFT $\quad 67$

7.1 Introduction . . . . . . . . . . . . . . . . . . . . . . . . . . . 67

7.2 Methods . . . . . . . . . . . . . . . . . . . 68

7.3 Results............................... 70

7.3.1 Ejection regimes

7.3.2 Jet ejection

7.4 Discussion . . . . . . . . . . . . . . . . . . . . 75

7.4.1 Effect of the pulse duration on the ejection mechanism

7.4.2 Radiation

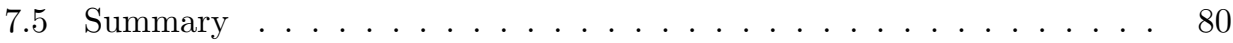

8 Towards 3D printing $\quad 83$

8.1 Introduction . . . . . . . . . . . . . . . . 83

8.2 Methods . . . . . . . . . . . . . . . . . . 84

8.3 Results . . . . . . . . . . . . . . . . 86

8.3.1 Microstructure of printed pillars

8.3.2 Repeatability of the printing process

8.3.3 Mechanical and electrical properties

8.3.4 Demonstrators and printing applications

8.4 Discussion . . . . . . . . . . . . . . . . . . . . . 90

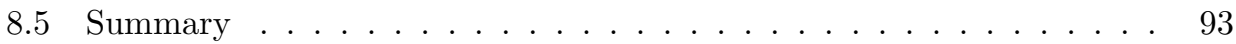

9 Solid phase LIFT of variable shapes $\quad 95$

9.1 Introduction . . . . . . . . . . . . . . . . . . . . . 95

9.2 Methods . . . . . . . . . . . . . . . . . . . 97

9.2.1 SLM beam shaping

9.2.2 Sample preparation and experimental methods

9.3 Results . . . . . . . . . . . . . . . . . . . . . . 101

9.3.1 Single-pulse process

9.3.2 Double-pulse process

9.4 Summary ............................ 106

10 Conclusions and recommendations $\quad 107$

10.1 Conclusions . . . . . . . . . . . . . . . . . . . 107

10.2 Recommendations . . . . . . . . . . . . . . . . 110

$\begin{array}{ll}\text { Bibliography } & 111\end{array}$

$\begin{array}{ll}\text { Acknowledgments } & 123\end{array}$ 



\title{
Nomenclature
}

\author{
Abbreviations \\ AFM \\ Atomic Force Microscope \\ BA-LIFT \\ Blister-Actuated Laser-Induced Forward Transfer \\ DMD \\ Digital Micromirror Device \\ DOS \\ Density of State \\ DRL \\ Dynamic Release Layer \\ FIB \\ Focused Ion Beam \\ IT \\ Inter-band Transition \\ ITT \\ Inter-band Transition Threshold \\ LIFT \\ Laser-Induced Forward Transfer \\ LITI \\ Laser-Induced Thermal Imaging \\ LMI \\ Laser Molecular Implantation \\ LTHC \\ Light To Heat Conversion Layer \\ MAPLE-DW \\ Matrix-Assisted Pulsed Laser Evaporation - Direct Write \\ SEM \\ Scanning Electron Microscopy \\ SHG \\ Second Harmonic Generation \\ SLM \\ Spatial Light Modulator \\ TTM \\ Two-Temperature Model \\ USLP \\ Ultra-Short Laser Pulses
}




\section{Greek Symbols}

$\alpha_{\text {bal }} \quad$ Ballistic motion absorption coefficient

$\alpha_{\text {opt }} \quad$ Optical absorption coefficient

$\alpha_{t h} \quad$ Thermal expansion coefficient

$\alpha_{\text {tot }} \quad$ Total absorption coefficient

$\Delta T_{l} \quad$ Lattice temperature difference

$\Delta T_{A X I S} \quad$ Temperature gradient along the optical axis

$\triangle T_{B E A M} \quad$ Temperature gradient perpendicular to the optical axis

$\Delta T_{e-l} \quad$ Temperature difference between the electrons and the lattice

$\Delta x \quad$ Resolvable displacement

$\Delta z \quad$ Mesh element size

$\delta_{D} \quad$ Thermal diffusion length

$\delta_{p i} \quad$ Mechanical deflection

$\eta \quad$ Viscosity

$\Lambda \quad$ Prefactor to determine electron pressure

$\lambda \quad$ Wavelength of the laser radiation

$\nu \quad$ Poisson's ratio

$\omega_{0} \quad$ Beam waist of a Gaussian beam

$\rho \quad$ Density

$\rho_{E} \quad$ Resistivity

$\sigma \quad$ Surface tension

$\sigma_{\text {opt }} \quad$ Transition cross-section

$\tau_{D} \quad$ Thermal diffusion time scale

$\tau_{p} \quad$ Laser pulse duration

$\tau_{e p} \quad$ Electron-phonon relaxation time

$\theta \quad$ Deflection angle of ejected droplets

$t_{0} \quad$ Break-up time of a liquid sheet

\section{Roman Symbols}

A

$A_{e}$

$A_{\text {srf }}$
Absorptivity

Specific heat constant of electrons

Surface area 
$c$

$C_{e}$

$C_{l}$

$C_{p}$

$C_{0}$

$C_{1}$

D

$d$

$D_{0}$

$d_{A P}$

$D_{\text {mod }}$

E

$E_{E}$

$E_{p}$

$E_{F}$

$E_{k i n}$

$E_{s}$

$E_{\text {vap }}$

F

$f$

$F_{A F M}$

$F_{\text {cap }}$

$F_{\text {jet }}$

$F_{\text {mod }}$

$F_{p l}$

$F_{\text {spray }}$

$F_{\text {val }}$

$g$

$h$

$h_{p i}$

$I_{0}$

$I_{\lambda}$

Speed of light

Heat capacity electrons

Heat capacity of the lattice

General heat capacity

Fitting factor for the elastic regime

Fitting factor for the vapor regime

Thermal diffusivity

Donor layer thickness

Impact droplet diameter

Aperture

Modification diameter

Young's modulus

Elastic energy

Laser pulse energy

Fermi level

Kinetic energy

Surface energy

Vapor energy

Laser peak fluence

Focal length

Force applied by the AFM

Cap ejection fluence

Jet ejection fluence

Modification threshold fluence

Plasma fluence threshold

Spray ejection fluence

Absorbed laser fluence

Electron-phonon coupling coefficient

Planck constant

Pillar height

Optical intensity of the incident laser pulse

Spectral irradiance 


\begin{tabular}{|c|c|}
\hline$I_{p i}$ & Moment of inertia \\
\hline$K$ & Bulk modulus \\
\hline$k_{B}$ & Boltzmann constant \\
\hline$K_{e}$ & Thermal conductivity of electrons \\
\hline$K_{l}$ & Thermal conductivity of the lattice \\
\hline$K_{e 0}$ & Electron heat conductivity constant \\
\hline$K_{t h}$ & Thermal conductivity \\
\hline$L$ & Probe distance \\
\hline$L_{m}$ & Latent heat of melting \\
\hline$L_{v}$ & Latent heat of vaporization \\
\hline$M^{2}$ & Beam quality factor \\
\hline$N$ & Carrier density \\
\hline$P$ & Probability of the type of ejections observed \\
\hline$P_{e}$ & Electron pressure \\
\hline$R$ & Reflectivity \\
\hline$r_{0}$ & Initial radius \\
\hline$R_{c}$ & Contact resistance \\
\hline$R_{p i}$ & Electrical resistance \\
\hline$r_{p i}$ & Pillar radius \\
\hline$S$ & Absorbed energy density \\
\hline$T$ & Transmittance \\
\hline$T_{e}$ & Electron temperature \\
\hline$T_{l}$ & Lattice temperature \\
\hline$T_{0}$ & Room temperature \\
\hline$T_{m}$ & Melting temperature \\
\hline$T_{v}$ & Vaporization temperature \\
\hline$u$ & Displacement of the lattice \\
\hline$V_{e j}$ & Ejection velocity \\
\hline$V_{i m}$ & Impact velocity \\
\hline$W e$ & Weber number \\
\hline$W e_{e j}$ & Ejection Weber number \\
\hline$W e_{i m}$ & Impact Weber number \\
\hline$z$ & Cartesian coordinate collinear to the optical penetration axis \\
\hline
\end{tabular}




\section{Chapter 1}

\section{Introduction}

This chapter provides an introduction to the field of micro-machining and additive manufacturing in view of a laser based process for printing of micron sized metal droplets. Moreover the research objectives and the outline of the thesis are discussed.

\subsection{Additive manufacturing on the micrometer scale}

Modern micro-machining offers several advantages in a wide field of applications, such as electronics, for chemical analysis or in the field of medicine. Therefore, several processes and techniques have been developed. Due to their great flexibility, laser-based processes have been widely applied. Laser-based processes are mostly used for selective material removal processes by means of ablation, which is commonly used for laser cutting and drilling as well as for surface texturing. However, laser-based additive manufacturing on the micro- and sub-micron scale is of growing relevance for many industrial applications such as microelectronics and integrated optics such as those found in lab-on-a-chip, 3D printing, and stacking of microchips. Along with the variety of demanding applications, a huge variety of different materials is to be transferred in these applications. Nearly all applications require or benefit from high resolution and high speed, while achieving high flexibility and full control of the quality, i.e. morphology and size, of the deposited materials. So far, several laser-based techniques were developed to address these challenges, where each process offers its specific advantages and disadvantages regarding flexibility, resolution and throughput.

Due to continuous progress in additive manufacturing technologies the fabrication of complex 3-dimensional structures is routinely achieved. The mainstream technologies can be divided into photopolymerization, powder bed fusion, sheet lamination or deposition, and printing and direct-write technologies [1]. In par- 


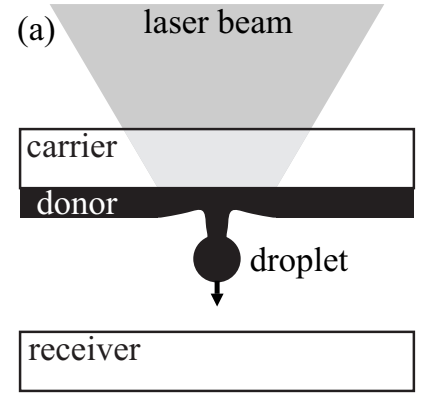

Figure 1.1: (a) Sketch of the LIFT process. (b) Ejected droplet deposited on the receiver.

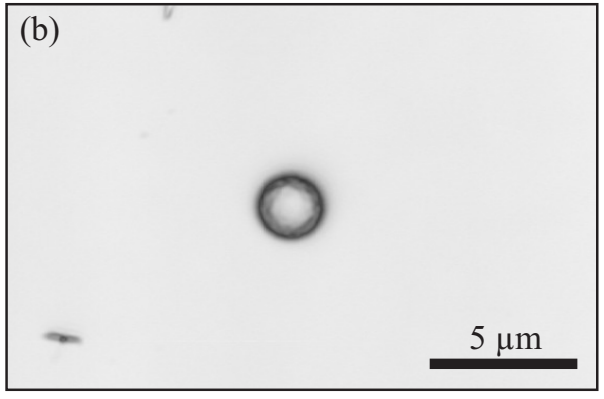

ticular the printing concept has gained momentum for rapid prototyping, since it provides for fast, low-cost, and contact-free deposition at room conditions and poses minimal disturbances to the receiver substrate (on which the material is deposited). For instance, deposition of wax, polymers [2], and even living cells [3] are now routinely achieved. However, printing of metals remains challenging, since the melting temperature of most metals is similar to (components within) the printing nozzle. As yet, metal printing has been limited to low-melting point metals $[4,5]$ and metal-containing inks [6-8], which are generally not optimized in terms of material properties (e.g. strength, conductivity, and corrosion rate) and cost. Contact-free deposition of a wider range of metals may therefore enable novel applications including optimized micro-antennas [9], electrode deposition on rough or inclined surfaces, or filling through-silicon vias for connecting stacked 2D electronic circuits [10].

\subsection{Laser-induced forward transfer}

Laser-induced forward transfer (LIFT) is a high-resolution 3D direct-write method that was first demonstrated in 1986 [11]. For the LIFT process, a transparent substrate (carrier) is coated with a thin film (donor) and is placed in close proximity to a second substrate (receiver), see figure 1.1 (a). A pulsed laser beam is focused through the carrier onto the carrier-donor interface. The incident laser pulse is absorbed within a thin layer of the donor material. At sufficiently high laser fluence levels, the donor material is ejected and deposited on a receiver substrate, as shown in figure 1.1 (b). LIFT has a high potential for printing of various materials, including pure metals [12-17], which cannot be deposited using conventional methods such as ink-jet printing, while retaining major advantages including high spatial resolution down to $300 \mathrm{~nm}$ [18]. Moreover, LIFT allows for a mask-less, contact-free deposition at room conditions. In particular, the deposition of pure metal droplets in the liquid phase allows for deposition of conductive patterns $[10,19]$, from which the semiconductor industry could strongly benefit [20]. 


\subsection{Research objectives}

Improving LIFT is proven challenging, as the ejection process is poorly understood and the exact ejection mechanisms are still under debate. Time-resolved imaging is a suitable method to visualize the ejection process and to gain further insight into the ejection process during LIFT. However, due to the challenging visualization conditions, time-resolved visualization has only been achieved for relatively thick liquid-film [21-25] and solid phase [26-28] or paste-transfer $[29,30]$ processes. Attempts to visualize LIFT-processing of $\mathrm{Au}$ [31], $\mathrm{Ni}$ [32], $\mathrm{Al}$ [33], and $\mathrm{Cr}$ [34] do not provide sufficient spatial resolution to track the process in detail. Therefore, theories describing the ejection mechanism have been proposed based on postprocess analysis of the craters left in the donor layer or deposited features on the receiver substrate [35-37]. In addition, numerical simulation has been performed [38-40]. Two driving mechanisms of the ejection process are commonly proposed [35, 36, 41]. First, relaxation of thermally induced stresses [42] could drive the ejection. Second, partial evaporation [41] of the donor layer, resulting in the formation of an expanding vapor bubble, may accelerate and eject the donor material. However, as yet, it is unknown under which conditions these ejection mechanisms occur. Therefore, several research objectives have been identified and will be studied in this thesis:

- Objective 1: Investigate the driving mechanisms that initiate the ejection process during LIFT.

Despite process improvements in various ways [43-47], the high potential of LIFT for liquid-metal deposition has not been met as the deposited features are poorly controlled. This for example results in deposition of one main droplet surrounded by smaller satellite droplets, the deposition of many particles [18], or a significant uncertainty in the deposition location due to a limited control of the ejection angle [48]. Therefore, a second research objective has been identified as:

- Objective 2: Investigate the ejection dynamics during LIFT that lead to the generation of undesired deposits on a receiving substrate.

Surprisingly, experimental studies on nanosecond [48] and femtosecond LIFT [42] indicate a similar ejection mechanism, which is characterized by the subsequent formation and break-up of a liquid jet for laser fluence values at the transfer threshold. However, an extensive description of the effect of the pulse duration on the ejection process during LIFT is still missing. The pulse duration is commonly discussed with respect to the minimal achievable droplet size and therefore often considered to be reduced to the generated heat affected volume in the donor layer [18]. However, the influence of the pulse duration on the ejection mechanism is not discussed as yet. Therefore, a third research objective has been identified as:

- Objective 3: Study the effect of the laser pulse duration on the ejection process during LIFT. 
So far, application of pure-metal LIFT for 3D direct-write has been limited to the deposition of single metal droplets [18, 42, 49], conductive lines or tracks [10, 19], and the deposition of nanoparticles [50]. This is because two challenging requirements have to be simultaneously fulfilled for consistent deposition: the impact location of a single droplet has to be limited to the previously deposited droplet's impact area, and good adhesion between the deposited droplets is required. For solid [35, 51] material transfer, which is mainly pursued to transfer intact parts of the donor film to the receiver, the adhesion is expected to be limited. Therefore, controlled deposition is often achieved in liquid phase. In this regime, for LIFT just above the ejection threshold fluence, a single spherical deposit is observed [18, 42, 49]. At intermediate ejection fluence levels, deposition of a torus-like shape is observed [18], which indicates that the droplet solidified in a spread-out state. At even higher fluence levels, a large amount of very small spherical droplets is observed [34, 52]. However, as stacking spheres or torus-shaped structures unavoidably results in limited drop-todrop contact and porosity after solidification, none of these shapes seems to be optimal for manufacturing high-density structures. Therefore, a fourth research objective has been identified as:

- Objective 4: Explore the droplet impact during LIFT and identify the crucial parameters with respect to $3 \mathrm{D}$ printing.

In the case of solid phase transfer, the ablation of a thin part of the donor layer near the carrier-donor interface [53] results in the delamination of the donor layer from the carrier substrate. Alternatively, thermally induced stress waves inside the donor layer [54] cause this delamination. So far, the transfer of thin films in solid phase remains challenging as the ejection process suffers from large shearing forces and requires full control over the laser fluence, as thin layers in the order of hundred nanometers easily melt. At the same time, applications ask for high flexibility regarding the shape of the deposited material. Several approaches have been demonstrated [55-59] in order to address the flexible printing of metals and metal pastes with variable shapes in solid phase. However, none of those approaches solves all the problems mentioned above. Therefore, the final research objective has been identified as:

- Objective 5: Explore the capabilities of LIFT for solid transfer of thin metal films of arbitrary shapes.

In order to achieve the formulated research objectives, an experimental approach supported by numerical modeling is employed. For the experimental investigation, post process analysis is supplemented with high-speed imaging techniques, which are found to be the method of choice. By means of the high-speed imaging, not only a qualitative impression of the ongoing ejection dynamics is captured, but also key properties such as the ejection velocity and directionality are obtained. The experimental data is interpreted on the basis of a two-temperature (TTM) model, which is commonly used for the interaction of ultra-short laser pulses with matter. The model is used to investigate the physical conditions of the donor layer, that ultimately lead to the observed ejection phenomena. Moreover, the impact and subsequent 
Research objective

Objective 1: Investigate the driving mechanisms that initiate the ejection process during LIFT.

Objective 2: Investigate the ejection dynamics during LIFT that lead to the generation of undesired deposits on a receiving substrate.

Objective 3: Study the effect of the laser pulse duration on the ejection process during LIFT.

Objective 4: Explore the droplet impact during LIFT and identify the crucial parameters with respect to $3 \mathrm{D}$ printing.

Objective 5: Explore the capabilities of LIFT for solid transfer of thin metal films of arbitrary shapes.
Addressed in chapter

$3,5,6,7$

$3,6,7$

$3,5,7$

8

3,9

Table 1.1: Summary of the formulated research objectives and the corresponding chapters that yield to the achievement of these objectives.

deposition of droplets is analyzed using a variety of post process analysis tools, which are interpreted with respect to the micro-structure, the mechanical and electrical properties of the deposited features.

\subsection{Outline of this thesis}

In addition to the brief introduction provided in this chapter, this thesis is divided into nine additional chapters. Chapter 2 provides an overview about prior work, related to the field of LIFT. To this end, a brief historical overview about LIFT based processes and their applications is given. Several reported results of different modeling approaches related to LIFT and the related results on high-speed imaging, i.e. monitoring of the transfer process are discussed. Chapter 3 addresses the numerical model that is used to investigate and support the understanding of the experimental results of the LIFT process. First a qualitative explanation of the relevant physics regarding the interaction of ultra-short laser pulses with metals is introduced. In a second step, the discrete two-temperature model, its implementation and validation is documented. Chapter 4 focuses on the experimental methods. A general description of the experimental setup used for the LIFT experiments throughout this thesis is presented. Moreover, details regarding the preparation of the samples and the tools for post-process analyzes are discussed. Chapters 5 to 9 address the formulated research objectives (summarized in table 1.1) and therefore present a detailed discussion in each chapter. Chapter 10 concludes the experimental findings and provides recommendation regarding further research and developments of the LIFT process. 



\section{Chapter 2}

\section{State of the art}

This chapter provides a brief overview about existing LIFT based transfer methods and presents a brief summary of relevant literature related to the fields of LIFT related modeling approaches and time-resolved imaging methods.

\subsection{Review on LIFT based processes}

In 1986 Bohandy et al. [11] demonstrated the feasibility of the LIFT process by depositing copper onto a silicon substrate. Bohandy's initial report introduced the first hypothesis of the material removal/ejection process. Based on this work, in 1987 Adrian et al. [60] studied the mechanism of the LIFT process and developed the first numerical model, which uses the finite difference method to investigate the ejection process. In the following years several alternative LIFT based processes were developed. These alternative processes mainly focus on reducing the droplet size [61], but also on transferring more complex, often more sensitive materials like biological materials or complete devices like micro-electronics [26]. Over the years, the flexibility of the LIFT process led to a growing interest of the research community on this topic, as indicated by figure 2.1. The following subsections provide a brief review about the variations of the different LIFT processes as they are commonly discussed in literature.

\subsubsection{Laser-induced forward transfer}

The original LIFT process is based on an ablation process, which results in a deposition of various types of materials [62, 63], mostly metals [11-13, 15, 49]. The setup is fairly simple and consists of the material which is to be transferred (donor material), a substrate on which the donor material is coated (carrier), and a second substrate on which the donor material is deposited (receiver). Figure 2.2 (a) shows a schematic of the LIFT process. To initiate the transfer process, a laser 


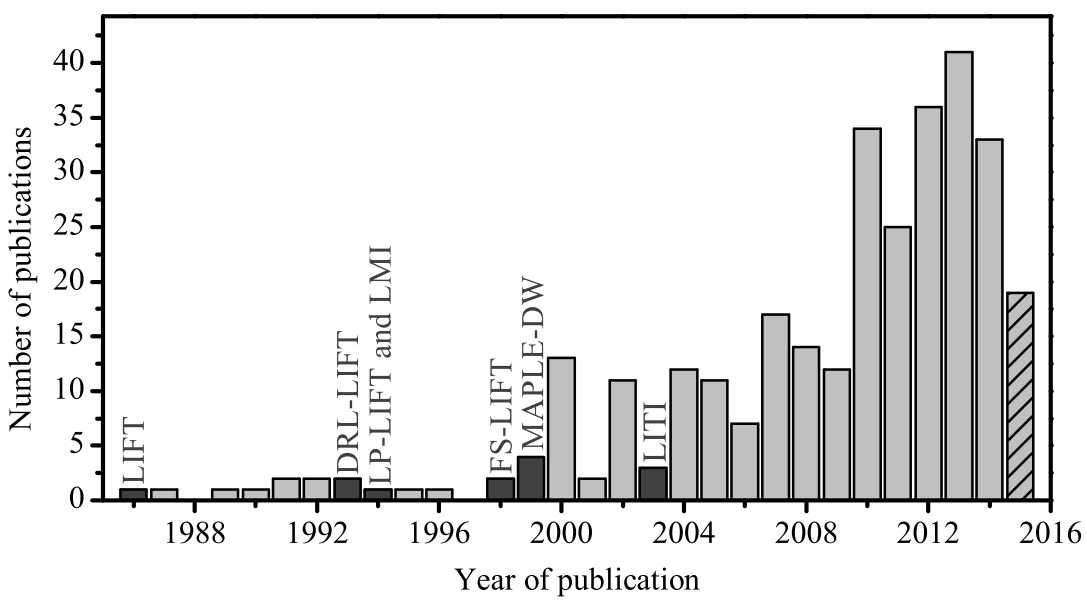

Figure 2.1: Overview of publications related to LIFT. Data obtained from Scopus in April 2015 (hashed). LIFT based processes are indicated accordingly.

beam is focused through the transparent carrier onto the carrier-donor interface. The incident laser beam is partially absorbed within a thin layer of the donor material. Depending on the experimental conditions, the donor material is melted and/or partially vaporized. Due to the thermally induced stresses [54, 64] and the arising pressure due to a potential vapor bubble [36, 41], the donor material is subsequently ejected and propelled towards the receiver, see figure 1.1. Volume, size and morphology of the deposited feature strongly depend on the processing parameters, as these determine the dominating physics triggering the ejection process. Using the LIFT technique deposited droplets as small as $300 \mathrm{~nm}$ in diameter have been achieved [18].

\subsubsection{Dynamic release layer LIFT}

The Dynamic Release Layer LIFT (DRL-LIFT) is an alternative to the original LIFT process and was proposed by Tolbert et al. [55]. The process aims at the transfer of more delicate materials, which must not be exposed to the incident laser pulse energy. Therefore, an additional sacrificial layer, referred as dynamic release layer (DRL) is added in between the carrier and the donor layer, see figure 2.2 (b). The ejection process is triggered, as the incident laser pulse is fully absorbed in the DRL, which leads to the full vaporization of that layer. Therefore, the donor remains unaffected and the pressure build-up leads to the transfer of the donor layer to the receiver. DRLLIFT is a highly flexible transfer process, which restrictions are mainly determined by the adaption properties of the involved materials. So far, various materials $[65,66]$ including polymers [67] as well as liquids [68] and living cells [69, 70] have been successful transferred. A drawback DRL-LIFT is given by the residual components of the DRL, that may contaminate the deposits on the receiver. 
(a) LIFT

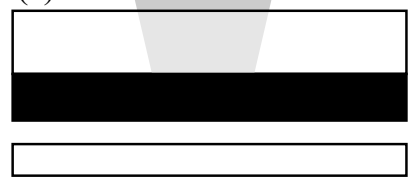

(d) LITI

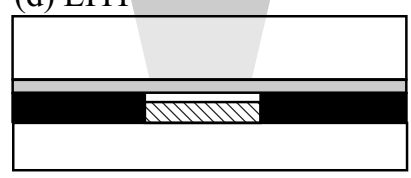

(b) DRL-LIFT

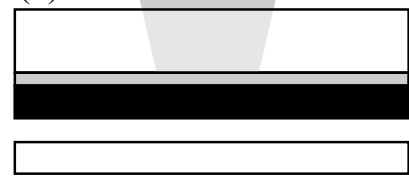

(e) LP-LIFT

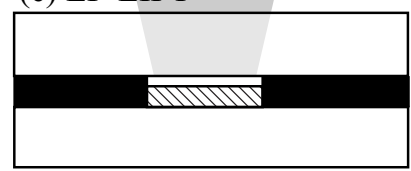

(c) MAPLE-DW

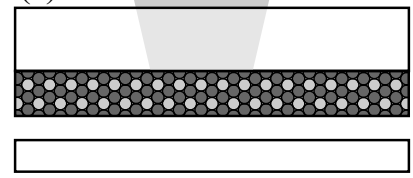

(f) LMI

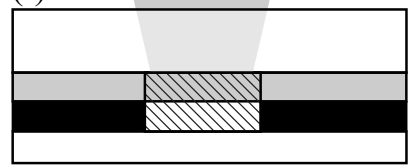

Figure 2.2: Sketches of the different varieties of the LIFT process.

\subsubsection{Matrix-assisted pulsed laser evaporation - direct write}

The Matrix-Assisted Pulsed Laser Evaporation - Direct Write (MAPLE-DW) technique, as proposed by Piqué et al. [43], combines the established MatrixAssisted Pulsed Laser Evaporation and the LIFT process, see figure 2.2 (c). It provides higher flexibility regarding the choice the of the donor material than the original LIFT process, as it limits the affect of the incident laser pulse on the donor material and therefore prevents the transferred donor from damage. This is achieved by embedding the donor material into a matrix, which is made of a different material than the donor material. The melting point of the matrix material is selected to be significantly lower than the melting point of the donor material. Similar to the DRL-LIFT process, the heat that is generated by the absorbed laser beam, only evaporates the additional matrix material, which subsequently releases the donor material and provides the thrust to transfer the remaining particles towards the receiving substrate. Due to the flexibility of MAPLE-DW a variety of materials, including metals [43] and cells [71] were successfully deposited.

\subsubsection{Laser-induced thermal imaging}

Laser-Induced Thermal Imaging (LITI) is a non-lithographic technique developed to transfer conducting polymeric material [45], as shown in figure 2.2 (d). Similar to DRL-LIFT, an additional layer is placed between the carrier and the donor, referred to as the Light To Heat Conversion Layer (LTHC). This additional layer is used to protect the donor from the incident laser beam. However, the LTHC is not supposed to be ablated, but is used as a indirect heat source, as it absorbs the incident laser pulse. For the transfer process, the donor and the receiving substrate are placed in contact. Next, the LTHC is exposed to the laser pulse and the heat build-up in the LTHC lowers the adhesion between the donor and the LTHC. Now, the adhesion force between the donor and the receiver dominates over the adhesion between the carrier and the donor and the donor sticks to the receiver, as the carrier is removed. Since there is no ablation involved, the contamination of the deposited material can 
be minimized. Besides a second additional layer can be added in between the LTHC and the donor layer to further protect the donor material.

\subsubsection{Long pulsed LIFT}

Long-Pulsed LIFT (LP-LIFT) represents yet another complementary LIFT process, see figure 2.2 (e). The experimental setup is similar to the common LIFT configuration, but instead of sub-nanosecond laser pulses, laser pulses in the order of microseconds or longer are used. For the LP-LIFT the donor layer and the receiver are in close contact. By heating the donor layer, the expanding donor material tightly contacts the receiver. Due to the long pulse durations used, the donor material is kept at high temperature for a relatively long time. The donor material anneals to the receiver and is bonded locally when the layers are separated. Kántor et al. [72] demonstrated the transfer of a $5 \mu \mathrm{m}$ tungsten segment in solid phase, which showed no evidence of melting. It was found that in addition to a fluence related threshold, there exists an additional threshold that is related to the pulse duration of the laser pulses applied. Pulse durations shorter than $500 \mu$ s did not lead to a transfer of material, which indicates the differences to the common LIFT process, where shorter pulse durations tend to provide better results. As it is based on annealing, this process in principle allows for the transfer of various materials in solid phase. However, the potential materials are restricted due to the thermal load caused by this process.

\subsubsection{Laser molecular implantation}

The Laser Molecular Implantation (LMI) has been demonstrated by Fukumura et al. [47] in 1994, see figure 2.2 (f). This transfer mechanism aims at the transfer of single dopant molecules instead of complete donor layers. The molecules are implanted in a thin polymer film (source) which is kept in close contact to the receiving layer. The material of the receiving layer is similar to the undoped source. The absorption of the incident laser beam depends on the doping concentration of the polymer, and increases with higher doping concentration. Contrary to the LIFT process, the source is not heated directly, but the absorbed energy is used to activate the dopant molecules. However, due to the interaction of activated dopant molecules and the surrounding polymer, the polymer is heated and expands afterwards. Subsequently, the activated molecules are released from the source and transferred to the undoped, receiving substrate. This technique has been demonstrated in forward- and backward-transfer geometries and was applied to transfer various materials [73, 74].

\subsubsection{Summary}

To this day, many alternatives to the original LIFT process were developed. The introduced list of process is not intended to be exhaustive. Nevertheless, an overview about the principles of the LIFT process has been given. Each process was developed to tackle a certain problem, which usually aims at the transfer of a new, 
often more delicate, donor material. Even though the experimental setups vary, the basic principles remain the same. All process are based on a locally confined laser induced activation or thrust build up to transfer the donor material. The variations of the setups are caused by the characteristic and the complexity of the material to be transferred. Ultimately, the choice of the best suitable transfer method depends on the application parameters and has to be made for each specific application independently.

\subsection{Modeling}

The ejection process is found to be a multidisciplinary phenomena, consisting of solid-state physics to describe the laser-matter interaction and complex fluid mechanics describing the subsequent droplet forming and ejection of the latter. As of yet, experimental observations do not allow for a complete understanding of the LIFT. Hence, numerous numerical investigations addressing certain steps of the ejection process have been employed to gain further insights into the ejection process during LIFT. This section provides an overview of relevant attempts to model the LIFT process.

\subsubsection{Review on modeling}

Seifert et al. [75-77] reported on numerical approaches aiming at the subsequent formation and ejection of liquid gold droplets using Navier-Stokes equations. Those investigations show the thermal and hydrodynamic behavior of a gold surface under pulsed laser irradiation. It has been shown that both, the formation and the desorption of small gold droplets are related to hydrodynamic effects driven by an equilibrium of inertial forces and surface tension. Accordingly, the desorption process was investigated and a minimal droplet size of $200 \mathrm{~nm}$ was calculated for an irradiated gold surface.

Willis et al. [78] introduced a two-dimensional axis-symmetric numerical model based on the Volume of Fluid (VOF) method. The model was developed to compute heat transfer, phase change, and fluid flow in the donor layer. The results were used to investigate the influence of volumetric expansion associated with the melting process on the surface deformations observed on several LIFT experiments. It was found, that the volumetric initiated fluid motion that was directed away from the carrier, was sufficient to induce deformations that remain after solidification. The theoretical findings were supported by experimental observations, which show a frozen droplet impingement for laser fluence levels below the transfer threshold.

Ivanov et al. [79] studied the formation of laser induced nanobumps caused by femtosecond laser pulses. Even though the model is not focusing explicitly on LIFT, but on thin films in general, it nicely describes the initial stage of the LIFT process. The model used in the simulations combines the classical Molecular Dynamics (MD) method for simulation of non-equilibrium processes of lattice superheating and fast phase transformations with a continuum description of the laser excitation and 
subsequent relaxation of the conduction band electrons based on a two-temperature model.

Banks et al. [80] employed a numerical interference model under consideration of the electric field of a femtosecond laser pulse and the gap size between the donor and the receiver. For sufficiently transparent donor materials (e.g. GdGaO), the dependency of the resulting intensity distribution was found to explain the observed variations in threshold fluence levels as well as variations of the morphology of the deposits.

Based on a two-temperature model Shugaev et al. $[54,81]$ investigated the heating and melting dynamics in different metallic films ( $\mathrm{Au}, \mathrm{Zn}, \mathrm{Cr})$ irradiated by femtosecond laser pulses. The TTM considers laser-generated stress waves, which where found to be key for the explanation of prior experimental observation, i.e. the transfer of donor in different physical states at similar laser conditions. It was pointed out that the ejection process is initiated by the tensile stresses, due to the reflection of the laser generated stress wave.

Röder et al. [41] employed numerical model to describe the underlying physical process during the LIFT process. Therefore, a finite difference simulation is used to predict threshold fluence levels as well as the "blow-off" times of thin nickel layers. Further, the model shows that the gasification of the carrier is the main driving force of the process.

Recent investigations on blister-actuated LIFT (BA-LIFT) were performed by Brown et al. [82]. Based on the finite volume (FVM) and the volume-of-fluid method (VOF), the driving mechanisms starting with the expansion of the laserinduced bubble, and the related fluid dynamics, which ultimately lead to an ejection of liquid ink, were analyzed. In addition, several ink properties related to the blistering process were investigated.

\subsubsection{Summary}

The review presented above is based on literature, directly and indirectly related to the modeling of LIFT. So far, the published models address the influence of the stress buildup, that is generated by the laser pulse and the fluid motion of the affected donor material. Because of the particular relevance of low fluence ejections during LIFT, current models focus on the physical conditions occurring at those fluence levels. Due to the complexity of the nucleation dynamics induced by ultra-short laser pulses, studies on the ejection process during high-fluence LIFT are still missing.

\subsection{Time-resolved imaging}

In order to gain insights into the details of the transfer characteristics during LIFT, time-resolved imaging techniques are employed. In principle, both high-speed cameras as well as pump-probe imaging techniques can be used. However, common highspeed cameras often suffer from limited spatial resolution in favor of high frame-rates. Due to this disadvantage, common high-speed cameras are not feasible to capture 
the LIFT ejection dynamics, with typical ejection velocities of up $2000 \mathrm{~m} / \mathrm{s}$ at the required spatial resolution in the single micrometer range. Therefore, pump-probe or laser-induced shadowgraphic imaging techniques are typically employed. Those experimental setups typically consist of a pump source which is chosen with respect to the process parameters of the LIFT process. The imaging is realized using a microscope in combination with a preferably incoherent probe source that is used to illuminate the scene. The spatial resolution is determined by the transfer function of the imaging system, i.e. the optical components used in the microscope and the sensor of the camera (CCD, ICCD, CMOS). The temporal resolution of a single measurement is determined by the pulse duration of the probe source, which is often provided by an additional laser source, often connected to an external fluorescence cell. Typically, the pump and the probe sources are electronically synchronized, allowing for a tunable time delay of sub-nanoseconds. Thus, time-resolved image sequences can be generated out of multiple ejections events, captured with a varying time delay. Along with the development of the LIFT process, the first materials were transferred without a so-called sacrificial or dynamics-release layer (DRL-LIFT, see section 2.1). Accordingly, the basic LIFT process was in focus of the first time-resolved imaging studies. The remainder of this section provides a review of relevant literature in the field of time-resolved imaging of the LIFT process.

\subsubsection{Review on time-resolved imaging}

Nakata et al. [31] investigated the LIFT process of thin gold films with layer thicknesses of $20 \mathrm{~nm}, 100 \mathrm{~nm}$, and $500 \mathrm{~nm}$, respectively. To this end, two-dimensional laser induced fluorescence (2D-LIF) was employed. The experiments were performed in atmospheric air and vacuum. A systematic study of the laser energy and the film thickness revealed ejections velocities of $2000 \mathrm{~m} / \mathrm{s}$ and $100 \mathrm{~m} / \mathrm{s}$ for single atoms and emissive particles, respectively. In addition, the experiments revealed a direct dependence of the angular divergence on the ablation energy and the film thickness.

Bullock et al. [33] performed an imaging study on the LIFT process of aluminum, using picosecond laser pulses and a shadowgraphic-interferometer imaging system. A systematic variation of the film thickness, laser pulse duration, and incident laser fluence revealed useful characteristics such as high directionality, high density, and sharply defined longitudinal spatial profiles. The experimental velocity data was numerically recovered by a proposed model that includes laser-induced avalanche ionization and multiphoton mechanisms, aiming at laser fluence levels in the breakdown regime.

Sano et al. [32] employed a shadowgraphic imaging study in order to investigate LIFT key parameters such as laser fluence and carrier-receiver separation with respect to the accuracy of the deposited features. Accurate printing was achieved using optimum laser fluence levels and a minimized carrier-receiver separation. In contrast, higher fluence values result in a spreading of the deposits, which was found to be related to a significant shock, occurring during impact. Time scales for the ejection process were achieved by pump-probe measurements, indicating a full layer removal within the pulse duration of the LIFT laser of 30 nanoseconds. 
Papazoglou et al. [83] investigated the dynamics of sub-picosecond LIFT of 200 $\mathrm{nm} \mathrm{InO}_{\mathrm{x}}$ films by means of time-resolved shadowgraphy. The LIFT process was investigated for time delays between 10 nanoseconds and 10 microseconds. The LIFT ejection was found to be highly directional, exhibiting an angular divergence of $3^{\circ}$ while achieving velocities of $400 \pm 10 \mathrm{~m} / \mathrm{s}$, depending on the laser fluence. Besides, the recorded images clearly show that the LIFT ejection is driven by a shockwave and most of the material is transferred in solid state, which is due to the pulse duration and the wavelength.

Feinaeugle et al. [29] investigated the transfer of solid phase material by femtosecond LIFT by means of time-resolved shadowgraphic imaging techniques. Ejection velocities of $V_{e j} \approx 48 \mathrm{~m} / \mathrm{s}$ and $V_{e j} \approx 34 \mathrm{~m} / \mathrm{s}$ for intact transfer of $d \approx 1.1$ $\mu \mathrm{m}$ thick $\mathrm{Bi}_{2} \mathrm{Se}_{3}$ and $d \approx 1.8 \mu \mathrm{m}$ thick PZT respectively, and of $V_{e j} \approx 140 \mathrm{~m} / \mathrm{s}$ for $d \approx 0.5 \mu \mathrm{m}$ thick Terfenol-D were reported. In contrast to prior observations, no shockwave was observed during the transfer. Due to the low ejection velocity and the absence of a shockwave, it is implied that femtosecond LIFT is suitable for intact transfer of solid materials.

Based on time-resolved shadowgraphy and scanning-electron microscope (SEM) images Unger et al. [84] captured femtosecond LIFT melt dynamics in $60 \mathrm{~nm}$ thick gold films with a laser fluence range below the ablation threshold. For laser fluence just above the transfer threshold, the formation of a liquid jet, and the subsequent separation of a gold droplet is documented. The recorded transfer dynamics are comparable to cavitation bubble dynamics near open surfaces in liquids and suggest the ejection process to be driven by the relaxation of thermally induced stresses. The proposed explanation is supported by SEM images that proof the existence of a fluid dynamic feature, called counter-jet. The ejection dynamics are happening at timescales of several 100s of nanoseconds, depending on the laser fluence applied.

\subsubsection{Summary}

The presented literature review above is not intended to be exhaustive, but covers the most important publications in the field of time-resolved imaging of LIFT of solid films. Still, only a few articles on the imaging of LIFT of pure metals exist. Unfortunately, these publications either suffer from limited spatial resolution [31-34] or miss a systematic study of experimental parameters. Hence, a detailed classification of different ejection regimes towards higher fluence levels is missing, for the ejection dynamics of copper as well as for gold. 


\section{Chapter 3}

\section{Laser-material interaction and model implementation}

In this chapter* an introduction to the interaction of ultra-short laser pulses with metals in the framework of LIFT is presented. The governing physics that describe the absorption of the laser pulse, the subsequent thermalization of a thin metal film and the resulting thermophysical properties, i.e. the build-up of thermally induced stresses and the related phase changes are discussed. Finally, a numerical model to simulate the thermal distribution in a thin metal film during LIFT is proposed.

\subsection{Interaction of ultra-short laser pulses with metals}

The response of metals on optical excitation is well described by Fresnel formulas employing complex index of refraction. Within a volume given by the optical penetration depth and the spot size of a focused laser beam, the absorbed laser energy leads to a thermalization of the irradiated sample. Depending on the laser intensities single or multi-photon absorption is triggered, which leads to an excitation of the electron subsystem. For continuous wave (CW) laser radiation typical excitation rates are much smaller than the subsequent relaxation through electron-electron and electron-phonon scattering. Hence, in the case of CW laser processing the electron- and phonon-subsystems are considered to be in thermal equilibrium and as a consequence, common heat diffusion dominates the heat dissipation into the sample. However, for ultra-short laser pulses (USLP)

* Parts of the work described in this chapter have been published in Physical Review Applied, 3:024001, 2015, Ralph Pohl, Claas Willem Visser, Gert-Willem Römer, Detlef Lohse, Chao Sun, and Bert Huis in 't Veld. Ejection Regimes in Picosecond Laser-Induced Forward Transfer of Metals. 


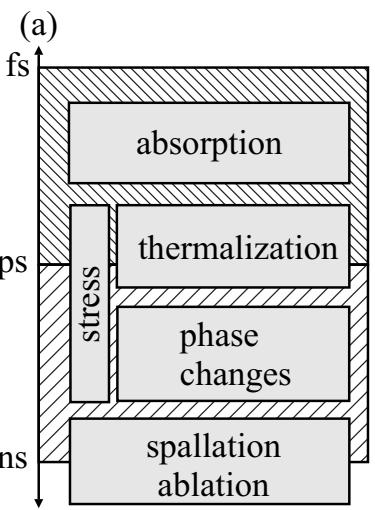

(b)

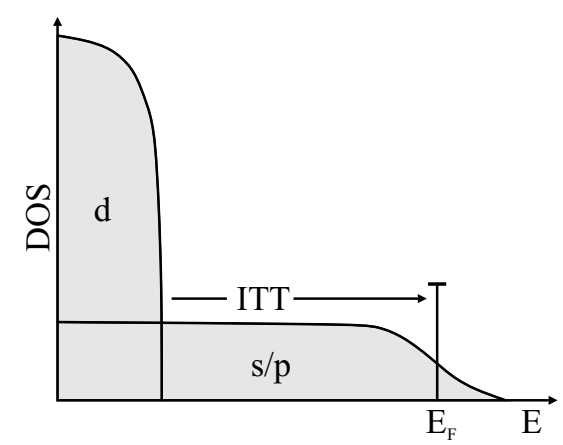

Figure 3.1: (a) Qualitative illustration of the excitation mechanism due to USLP excitation in metals [85]. (b) Illustration of the electronic structure of $s / p$ band metals, explaining the inter-band transition [86].

in the (sub-)picosecond regime, the assumption of thermal equilibrium between the electron- and phonon-subsystem is no longer valid. An overview of typical timescales of the phenomena and excitation processes taking place during and after irradiation of a metal with an ultra-short laser pulse is shown in figure 3.1 (a). This section provides a brief description of these phenomena as well as of the relevant physics regarding the interaction of metals with ULSP. The topics of

- laser pulse reflection and absorption,

- the subsequent thermalization process of the metal sample,

- and the corresponding thermal physical phenomena, i.e. stress build-up and phase changes

are addressed in a chronological order.

\subsubsection{Reflection and absorption in metals}

Reflection: For metals the reflection of the incidence laser pulse at the surface of the bulk material is mainly determined by the electronic structure of the corresponding metal. Except for noble metals, most metals are dominated by the d-band excitation, upon laser irradiation. The excitation of such quasi-free electrons is well described by the mathematical description of harmonic oscillators, formulated by the Drude model. A derived key property for each material is given its plasma frequency. For light below the plasma frequency, the light is almost completely reflected. For light with a frequency above the plasma frequency, the light is only partly reflected and consequently partial absorption within the material takes place. Usually, the reflectivity of the metal surface is considered to be independent from variations in the electron temperature as resulting shifts of the Fermi distribution 
will not directly affect the occupancy of the involved states of transition. However, for noble metals such as copper and gold the electronic structure is described by a d$\mathrm{s} / \mathrm{p}$ band distribution, as outlined in figure $3.1(\mathrm{~b})$. The $\mathrm{s} / \mathrm{p}$ band structure provides additional occupancies, expressed as inter-band transitions (IT) between the $\mathrm{d}$ band and the $\mathrm{s} / \mathrm{p}$ band. As consequence, a broadening of the Fermi distribution due to increased electron temperatures therewith directly affects the electronic occupancy. As a consequence, decreased and increased reflectivities are observed for photon energies below and above the inter-band transition threshold (ITT), respectively. Those changes of the reflectivity have been computed on the basis of models proposed by Jah et al. [87] and experimentally determined for femtosecond laser pulses by Hohlfeld et al. [86]. However, for the wavelength used in this work $(515 / 532 \mathrm{~nm})$ the relative change of the reflectivity is expected to be in the order of single digit percentages $(<5 \%)$, and will therefore be neglected in further calculations presented in this work.

Absorption: The optical penetration depth is used to describe the penetration of the non reflected radiation of an incident laser pulse with the intensity $I_{0}$ into the material. The attenuated intensity

$$
I(z)=I_{0} \exp \left(-z N \sigma_{o p t}\right)=I_{0} \exp \left(-z \alpha_{o p t}\right)
$$

follows an exponential decay which depends on the penetration depth $z$ into the material, the cross section $\sigma_{o p t}$, and the density of electrons $N$ involved in the absorption process. The optical penetration depth is defined as $\frac{I(z)}{I_{0}}=\mathrm{e}^{-1}$ and can be obtained from the linear absorption coefficient $\alpha_{\text {opt }}$. For ULSP the optical penetration depth is not sufficient to ultimately describe the unhampered penetration of excited electrons into the bulk. To that end, a mechanism referred as ballistic motion of electrons needs to be considered. This motion is commonly considered via a modified absorption term

$$
\alpha_{t o t}=\alpha_{o p t}+\alpha_{b a l}
$$

which leads to an increased penetration depth. This approach is valid, as long as the penetration depth is significantly shorter than the thickness of the irradiated material under consideration. For thin copper and gold films, with a layer thickness of less than $100 \mathrm{~nm}$, the penetration depth exceeds the layer thickness and the radiation is only partially absorbed within the layer. Therefore, multiple internal reflection theory needs to be considered to describe the effective absorption in a thin film. For further calculations, the absorbed energy density $S$ will therefore be described by

$$
S(r, t)=\frac{\alpha_{t o t} A I(r, t) \exp \left(-z \alpha_{t o t}\right)}{1-\exp \left(-d \alpha_{t o t}\right)}
$$

with $d$ the thickness of the film and $A=1-R-T$, where $R$ and $T$ denote the reflectivity and transmissivity, respectively. 
(a) nonequilibrium

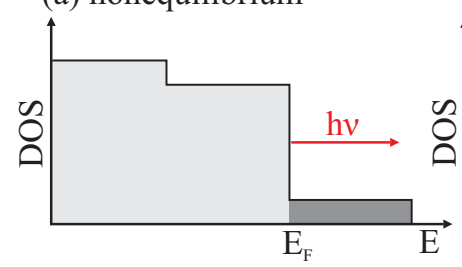

(b) $\mathrm{T}_{\mathrm{e}}>\mathrm{T}_{1}$

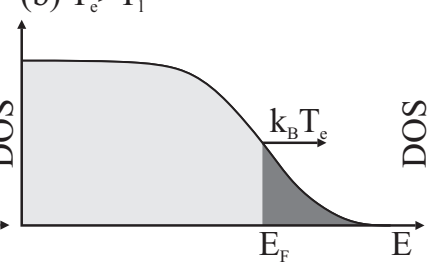

(c) $\mathrm{T}_{\mathrm{e}}=\mathrm{T}_{1}$
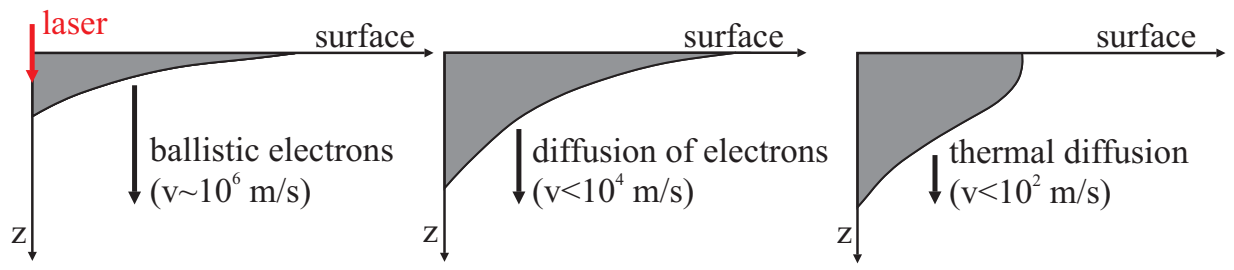

Figure 3.2: Optical excitation mechanism induced by ultra-short laser pulses, adapted from [86]. (a) Photon absorption within the optical penetration depth. (b) Thermalization of the electron subsystem. (c) Thermalization of the electron and phonon subsystem.

\subsubsection{Thermalization}

The thermalization process of the lattice due to USLP is best described by a formulation of subsequent scattering processes within and between the electronand the phonon-subsystems. The energy transfer is therefore determined by the scattering rate and the amount of energy that is transferred at each scattering event. The transferred energy is thereby related to the mass balance of the scattering partners involved. Due to the mass difference between the electrons and the phonons, the energy transfer between the electron- and the phonon-subsystem happens on timescales of picoseconds, whereas the thermalization of the electron gas due to electron-electron scattering takes place in the femtosecond regime. As the consequence a complex excitation process needs to be considered for the absorption of USLP, which is summarized in figure 3.2.

Photon absorption: The absorption of the incident laser pulse (described by photon-electron scattering) leads to a direct excitation of the electron-subsystem, see figure 3.2 (a). The electrons are heated up to a strong non-equilibrium state. In this phase two competing processes, namely the ballistic motion and the thermalization of the electron gas take place. The ballistic motion describes the unhampered penetration of the heated electrons into the bulk material. The electrons travel with velocities given by the Fermi velocity into the bulk material. The ballistic range is then given by the Fermi velocity and the time-scale needed for the electronic subsystem to reach thermal equilibrium. Typical values obtained from time-resolved measurements are in good agreement with the ballistic range estimated from the mean free path length of heated electrons. As a consequence, the ballistic range becomes dominant for noble metals, as the electrons experience a lower density, and 
therewith lower scattering rate, i.e. a longer mean free path length. For copper and gold, typical values are found to be $117 \mathrm{~nm}$ and $70 \mathrm{~nm}$, respectively. It is important to note that these values were determined for femtosecond laser pulses and vary across literature. For pulse durations of picoseconds the effect of ballistic motion is generally expected to be less pronounced when compared to femtosecond laser pulses and are therefore often neglected.

Electron thermalization: Due to the ongoing scattering processes, the thermalization of the heated electron subsystem typically takes place within a few $10 \mathrm{~s}$ of femtoseconds. Within this timescale, the initially deformed density of states (DOS) distribution recovers to form ultimately a Fermi distribution of DOS, see figure 3.2 (b). A well defined electron temperature is established, and diffusive energy transport within the electron gas dominates the heat dissipation. A detailed theoretical investigation on distorted DOS and its affects on the physical properties has been presented by Rethfeld et al. [88]. It was demonstrated that the optical excitation by a femtosecond laser pulse leads to a deformed DOS distribution, that results in a variation of the electron-phonon coupling factor $g$. The latter is a commonly used parameter for the scattering rate between the electrons and the phonons.

Electron-phonon thermalization: Once the electron subsystem reaches equilibrium conditions, ongoing scattering processes between the electrons and phonons ultimately lead to a thermal equilibrium between the electron- and phonon subsystems, see figure 3.2 (c). The timescale on which the energy is transferred from the electrons to the lattice is described by the electron-phonon coupling factor. In this phase, common thermal diffusion within the lattice is considered. Due to the unbalance between the heat capacities of the phonons and the electrons, the temperature rise of the phonons is significantly smaller than for the electrons and the corresponding temperature gradient in the phonon system into deeper parts is relatively small. As a consequence, the heat transport is dominated by the electron temperature gradient, hence the electron subsystem. For this reason, heat conduction in the lattice of metals is often neglected.

\subsection{Thermophysical phenomena}

Once the laser energy is absorbed in the metal film, different thermophysical phenomena, namely the generation of thermally induced stresses and phase changes of the initially solid metal take place. This section provides a brief overview of these phenomena.

\subsubsection{Stress generation}

Thermally induced stress build-up in thin layers, induced by USLP, is widely discussed in literature but the details are still part of ongoing discussions. A 
common theory of stress build-up is based on the two-temperature thermoelasticity [89], which treats electrons and the lattice as two different subsystems. Accordingly, two main contributions to laser induced stress build-up are often discussed, namely: hot-electron blast force and the stress related to the classical thermal load. The propagation of the generated stress wave [54] can be derived from the lattice displacement $u$ expressed as

$$
\rho \frac{\delta^{2} u}{\delta t^{2}}=E_{1} \frac{\delta^{2} u}{\delta z^{2}}-E_{2} \alpha_{t h} \frac{\delta T_{l}}{\delta z}+2 \Lambda\left(T_{e} \frac{\delta T_{e}}{\delta z}\right),
$$

in which $T_{e}, T_{l}$, and $t$ correspond to the electron temperature, the lattice temperature and the time, respectively. Here, $\rho$ represents the material density and $\alpha_{t h}$ is the linear expansion coefficient. The parameter $\Lambda$ is related to the density of states at the Fermi surface and is commonly used to determine the relation between the electron pressure $P_{e}$ and the electron temperature $P_{e}=\Lambda T_{e}^{2}$. The parameters $E_{1}$ an $\mathrm{d} E_{2}$ are related to the Young's modulus $E$ and the Poisson's ratio $\nu$ and are determined as

$$
E_{1}=\frac{E(1-\nu)}{(1+\nu)(1-2 \nu)}, E_{2}=\frac{E}{(1-2 \nu)} .
$$

Hence, in equation (3.4) it is reflected that both the electron- and phonon-subsystems expressed by the electron temperature and the lattice temperature, contribute to the generated stress. That is, the stress build-up is obviously dominated by the temperature gradients of the electron and phonon subsystems.

Electron blast: A general description of the subsequent stages, i.e. the local displacement of the lattice as a result of laser excited electrons is given in the following. The initial state of the material consists of unperturbed atoms performing harmonic oscillations around equilibrium conditions. The atoms proceed the unhampered oscillations, while the electron subsystem is subsequently thermalized by the incident laser pulse. An electro-static force that is related to the electronic pressure and the temperature gradient in the electron subsystem, induces atomic motion in the lattice, referred as "blast force". Typically, this blast force is established within timescales of about 100 femtoseconds, resulting in a fast lattice displacement, i.e. fast stress build-up. Chen et al. [90] identified that the duration of this stage lasts up to several picoseconds. The magnitude of this force is proportional to electron temperature gradient. The shock wave generated by the blast force superimposes on the thermal load resulting from the nonuniform lattice temperature at later stages. As a result, changes in the micro-structural and mechanical properties of the material even at lattice temperatures far below the melting point have been observed.

Thermal stress: During the time interval related to non-equilibrium electron and lattice temperatures, the generation of a stress wave within the optical and or ballistic penetration depth takes place. The generation of the stress wave is attributed to the rapid heating of the lattice, with typical timescales driven by the 
electron-phonon scattering process, which is in the order of 10 s of picoseconds. In the final stage, the electron and phonon-subsystems are in equilibrium. However, due to the inhomogeneous temperature distribution, a significant temperature gradient along the optical axis exists, which leads first to further dissipation of the heat (see section 3.1.2) and secondly induces thermal strain on the lattice. Depending on the experimental conditions, stress values in the order of $10 \mathrm{~s}$ of GPa have been reported, for laser irradiated thin films [54].

\subsubsection{Phase changes and laser-induced breakdown}

In the following a brief introduction to the complex mechanism of laser-induced phase changes, as well as a short summary of laser-induced break down, i.e. the generation of a laser induced plasma is provided.

Superheating: Typically the thermalization of a thin metal film due to electronphonon scattering takes place on the timescale of 10s of picoseconds. However, due to the nucleation kinetics, the processes of melting and vaporization occurs on typical timescales varying from single to several 100s of picoseconds. Due to the rapid heating rates induced by USLP the lattice temperatures can therefore significantly exceed equilibrium temperatures before any phase change has occurred and the material enters a meta-stable state, referred as superheated. Typical values of the material temperature above the equilibrium phase change temperature, for a laser irradiated gold film by USLP, are about $250 \mathrm{~K}$ and $600 \mathrm{~K}$ of superheat for melting and vaporization, respectively [91]. Depending on the degree of superheating, two different nucleation mechanism dominating the phase changes are commonly considered. Namely, heterogeneous and homogeneous melting and vaporization.

Heterogeneous melting/vaporization: Modeling the laser-induced melting process is a challenging field of laser material interaction. To describe the process of heterogeneous nucleation at rather low degrees of superheating, models based on the wave hypothesis are employed. To this end, the kinetics of melting are sufficiently described by introducing a melt/vapor front which propagates through the layer. Those melt and vapor fronts are supposed to start at the surface of the laser irradiated film, propagating through the layer with maximum velocities in the order of about several $100 \mathrm{~s} \mathrm{~m} / \mathrm{s}$ for the melt and of about $10 \mathrm{~s}$ of $\mathrm{m} / \mathrm{s}$ for the vapor front, respectively. Hence, the time for melting and vaporization of a thin film is given by the fraction of the layer thickness and the melt/vapor front speed. Based on this mechanism, a typical timescale to fully melt a $100 \mathrm{~nm}$ thick gold layer is in the order of 100 picoseconds [85].

Homogeneous melting/vaporization: For higher degrees of superheating, a process referred as homogeneous nucleation needs to be considered. This process is often considered for explosive boiling phenomena, which is triggered by the huge heating rates of USLP and the volumetric heating given by the optical and ballistic penetration depth and the geometry of the focused laser spot. As 
a result, a volume of the lattice is heated up to a meta stable state with a high degree of superheating at a nearly single instance. Studies on this topic [92] have found melt/vaporization times of the whole volume in less than one picoseconds, resulting in a uncontrolled explosive boiling, also known as phase explosion. As a consequence, the homogeneous phase change is dominated by the timescale given by the scattering mechanism related to the thermalization of the lattice.

Laser-induced breakdown: For high laser fluence levels, an excited hot vapor, referred to as plasma occurs. This plasma consists of atoms, ions, electrons and excited states of the latter. Along with the excitation mechanism, the emissions of plasma specific radiation as well as a characteristic acoustical shock wave is generated by the rapid, high-velocity expansion of the plasma plume. The formation of laser-induced plasmas is quite complex and consists of a variety of physical phenomena, such as heating, melting, vaporization, atomization, excitation and ionization. Especially, the emitted radiation is of major interest, as it is strongly related to the materials involved, and is therefore exploited by laser-induced breakdown spectrometry. However, the interaction of USLP with solids with respect to plasma generation is still under investigation as it depends on multiple process parameters, such as laser pulse energy, pulse duration, laser wavelength, ambient atmosphere as well as on the chemical and physical properties of the specimen. In the frame work of this thesis, the plasma build-up is only briefly discussed and limited to one key parameter, which is given by the minimal fluence, that is needed to achieve laser-induced break down of the material. This laser fluence threshold is obtained from an estimated fluence that is required to achieve local vaporization of an irradiated surface [93]. Hence, the threshold can be estimated by,

$$
F_{p l}=\rho L_{v} \sqrt{D \tau_{p}}
$$

with $\rho$ the density of the material, its thermal diffusivity $D=K_{t h} /\left(\rho C_{p}\right)$, and the laser pulse duration $\tau_{p}$. In the case of thin film heating, as considered for the LIFT process, the threshold can be more accurately calculated by,

$$
F_{p l}=d \frac{C_{p} \Delta T+\rho\left(L_{m}+L_{v}\right)}{1-R}
$$

with the temperature difference $\Delta T$, the density $\rho$, the latent heat of melting $L_{m}$, the latent heat of vaporization $L_{v}$, the heat capacity $C_{p}$ and the optical reflection coefficient $R$.

\subsection{Model implementation}

In this section the numerical two-temperature model, that is employed to investigate the governing physics during the LIFT process is introduced. Two-temperature models have been widely applied to investigate the thermal response of thin metal films heated by USLP, as qualitatively described in section 3.1. The subsequent 


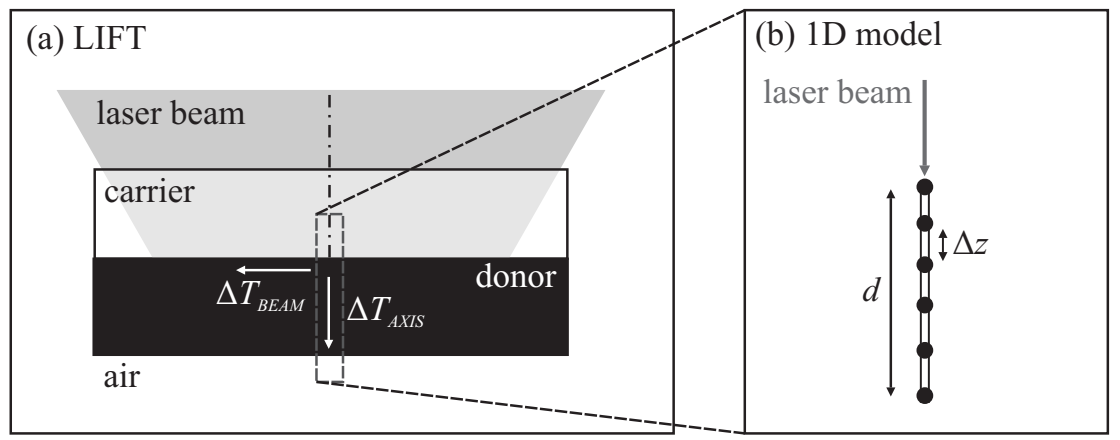

Figure 3.3: (a) Illustration of the LIFT process, indicating the generated temperature gradients along the optical axis $\left(\Delta T_{A X I S}\right)$ and the radial temperature gradient $\left(\triangle T_{B E A M}\right)$ caused by the energy density profile of the focused laser pulse. (b) Geometry and meshing of the 1D numerical model, indicating the donor layer thickness $d$ and the mesh element size $\Delta z$ that was applied. Here, only the donor layer is modeled and both, the carrier and the ambient air, are disregarded from the simulations, but incorporated via the boundary conditions.

thermalization processes related to the electron and lattice subsystems were first theoretically described by Kaganov et al. [94]. Thereon, Anisimov et al. [95] formulated a phenomenological, parabolic, two-step model to describe the electron and lattice heating dynamics in more detail. To account for the duality of the thermalization process, the electron and phonon subsystems are treated as individual heat reservoirs. In this approach, the scattering processes are represented by the electron-phonon coupling factor, which accounts for the energy exchange between those heat reservoirs. The absorption of the laser pulse, typically confined by the optical penetration depth (see section 3.1.1), is considered by a direct excitation of the electron subsystem. As of today, a variety of improvements of the TTM have been made, leading to more complex models, that include further physics such as: radiation losses, the generation of laser-induced stress waves, and detailed nucleation dynamics. The model introduced in this thesis is build on the basis of recent publications in the field of LIFT and thin film heating. The model is employed to investigate the physical conditions of the donor layer, that ultimately lead to the observed ejection dynamics during the LIFT process. Therefore the model includes the heating dynamics and a simplified description of the nucleation dynamics, i.e. melting and vaporization of the donor layer at equilibrium temperatures.

\subsubsection{One-dimensional approach}

The heat conduction in a heated film (see figure 3.3 (a)) is determined by the laser-induced temperature gradients, which ultimately determines the heat flow, i.e. energy transfer within the film. To simplify the model and speedup the numerical calculations, a one-dimensional approach is commonly used. Such an approach is 
often considered for thin film heating, and is justified by the geometry of the laser spot size relative to the thermal penetration depth, typically given by the sum of the optical penetration depth and the ballistic range [96]. To verify this approach for the heating of a $200 \mathrm{~nm}$ thick film of copper, a simple estimate is considered here. The thermal penetration depth $\delta_{D}$ into copper equals

$$
\delta_{D}=2 \sqrt{D \tau_{D}}
$$

with $D=1.1 \cdot 10^{-4} \mathrm{~m}^{2} / \mathrm{s}$ the thermal diffusivity of copper and time $\tau_{D}$ since the start of the laser pulse. For a given time interval of $\tau_{D}=100 \mathrm{ps}$, it is found that the given laser spot size exceeds the thermal penetration depth $\left(\delta_{D} \approx 200 \mathrm{~nm}\right)$ by two orders of magnitude. Hence, the temperature gradient coaxial with the optical penetration depth dominates the heat flow and heat conduction perpendicular to the optical axis $z$ can indeed be neglected. A sketch of the geometry of the one-dimensional numerical model is shown in figure $3.3(\mathrm{~b})$.

\subsubsection{Two-temperature model}

As discussed in section 1.3, this thesis studies picosecond and nanosecond LIFT. Though the proposed model can easily be extended to cover both timescales, the focus with respect to numerical temperature simulations is set to the picosecond regime. Pulse durations in this regime are comparable to the timescale of the electron-phonon relaxation time [97]. Therefore, a two-temperature model must be used to describe the heating dynamics of an irradiated film. The spatial and temporal evolution of the electron temperature $T_{e}$ and the lattice temperature $T_{l}$ are obtained by the following set of differential equations [54]

$$
\begin{gathered}
C_{e} \frac{\delta T_{e}}{\delta t}=\frac{\delta}{\delta z} K_{e} \frac{\delta T_{e}}{\delta z}-g\left(T_{e}-T_{l}\right)+S, \\
C_{l}\left(T_{l}\right) \frac{\delta T_{l}}{\delta t}=g\left(T_{e}-T_{l}\right),
\end{gathered}
$$

where $g$ corresponds to the electron-phonon coupling factor, and $C_{e}$ and $C_{l}\left(T_{l}\right)$ represent the heat capacities of the electrons and the lattice, respectively. Here, $C_{e}=A_{e} T_{e}$, where $A_{e}$ is the electron specific heat constant. As already described in section 3.1.2, the thermal conductivity in metals is dominated by the electronic structure and therefore given by the electron thermal conductivity $K_{e}=K_{e 0} T_{e} / T_{l}$. Compared to the heat conduction in the electron gas, the heat conductivity in the lattice is negligible and $K_{l}$ is set to zero and the corresponding diffusive term in equation (3.10) is omitted.

\subsubsection{Absorption of the laser pulse}

The temporal evolution of the incident laser pulse intensity $I_{0}(t)$, is shown in figure 3.4 (a). Mathematically this temporal evolution is described by

$$
I_{0}(t)=\frac{2 F \sqrt{\ln 2}}{\sqrt{\pi} \tau_{p}} \exp \left(-4 \ln 2\left(t / \tau_{p}\right)^{2}\right),
$$




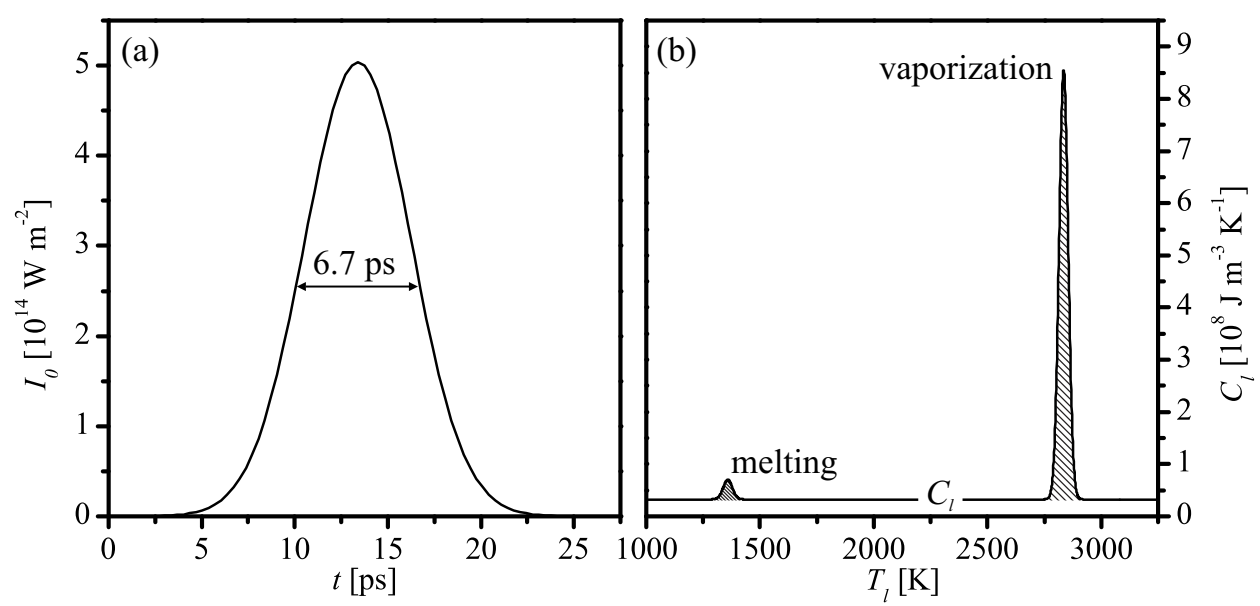

Figure 3.4: (a) Temporal evolution of the laser pulse, as defined by equation (3.11).(b)Variation of the lattice heat capacity as a function of the lattice temperature. The integrated hashed areas correspond to the enthalpies of melting and vaporization, centered at the equilibrium temperatures of melting and vaporization.

where, $F$ and $\tau_{p}$ are the peak laser fluence and the pulse duration of the incident laser pulse, respectively. Further, the peak fluence reads as

$$
F=\frac{2 E_{p}}{\pi w_{0}^{2}}
$$

with $w_{0}$ and $E_{p}$ being the beam waist and the pulse energy of the focused laser pulse, respectively. Further, the source term $S(z, t)$ is used to describe the electron heating of a thin film by Lambert-Beer absorption of the incident laser pulse, see section 3.1.1. Finally, incorporating multiple internal reflections in the film [86], the temporal and spatial evolution of the incident laser pulse is mathematically expressed as

$$
S(z, t)=\frac{\alpha_{t o t}(1-R) I_{0}(t) \exp \left(-z \alpha_{t o t}\right)}{1-\exp \left(-d \alpha_{t o t}\right)}
$$

where $R$ denotes the reflection coefficient and $\alpha$ the linear absorption coefficient.

\subsubsection{Initial and boundary conditions}

The initial conditions are defined by the initial electron and lattice temperatures. Both temperatures are initially in equilibrium and set to standard room temperatures $T_{e}=T_{l}=293 \mathrm{~K}$. Next, the boundary conditions at the carrier-donor and the donor-air interface need to be defined. A comparison of the thermal diffusivity of the donor layer material (copper $\approx 10^{-4} \mathrm{~m}^{2} / \mathrm{s}$ ) with respect to the carrier material (soda-lime glass $\approx 10^{-6} \mathrm{~m}^{2} / \mathrm{s}$ ) and the ambient atmosphere (air $\approx 10^{-5} \mathrm{~m}^{2} / \mathrm{s}$ ) readily justifies a zero flux boundary condition at both interfaces, as the thermal diffusivity of donor layer 
(copper) dominates the heat conduction. For this reason, both the carrier material and the ambient atmosphere are discarded from the geometry of the numerical model. Therefore, heat conduction at both interfaces $(z=0$ and $z=d)$ is neglected and zeroflux boundary conditions given by $C_{e} \frac{\delta T_{e}}{\delta t}=0$ are applied.

\subsubsection{Thermophyscial properties}

A qualitative description of the complex nucleation kinetics, related to melting and vaporization has already been discussed in section 3.2.2. Superheating might occur, resulting in melting timescales up to 100 ps [92]. Since these timescales are still significantly shorter than the timescale of donor ejections in LIFT experiments, temperature homogenization is expected to occur prior to ejection. Therefore, superheating is ignored in the temperature model and the thermophysical properties related to the phase change of the material are solely represented by the enthalpies of melting and vaporization. Those enthalpies are considered in the heat capacity of the lattice $C_{l}\left(T_{l}\right)$ as it is implemented as a function of the actual lattice temperature, see figure 3.4 (b). Therefore, Gaussian functions are superimposed to the fixed thermal heat capacity $C_{l}$ of the lattice. The integral of those functions yields the phase change enthalpies and allows to account for the energy losses, related to the phase changes. These functions are centered at the equilibrium phase change temperatures $T_{m}$ and $T_{v}$, exhibiting a standard deviation of $20 \mathrm{~K}$, for melting and vaporization, respectively. The spread of these Gaussian functions is necessary to achieve numerical stability of the model. The proposed mechanism of phase change is considered to be instantaneous in this temperature model, as no further nucleation kinetics have been included.

\subsubsection{Material parameters}

In the framework of this thesis the LIFT of two materials, namely copper and gold are investigated. The material properties that are used for numerical simulations are summarized in table 3.1. The data is for solid phase and was taken from Wellershoff et al. [97] and Hohlfeld et al. [86]. For the liquid and gaseous phase, only the phase change temperatures at equilibrium conditions and the corresponding enthalpies are considered.

\subsubsection{Numerical solver}

The two-temperature model is described by a set of hyperbolic, two-step heat conduction equations, see equations (3.9) and (3.9). Due to the strong nonliterary of these coupled equations and due to the additional temperature dependence of the introduced thermophysical material properties along with the volumetric heat source, these equations can hardly be solved using a general explicit solver. Therefore, an implicit solver is employed. Implicit solvers are commonly used to address the solution of this kind of diffusion dominated simulation. To this end, a commercial finite-element software package COMSOL is used. For the presented 


\begin{tabular}{lllll} 
Quantity & Symbol & Copper & Gold & Unit \\
Melting temperature & $T_{m}$ & 1358 & 1337 & $\mathrm{~K}$ \\
Vaporization temperature & $T_{v}$ & 2835 & 2800 & $\mathrm{~K}$ \\
Lattice heat capacity & $C_{l}$ & $3.26 \cdot 10^{6}$ & $2.19 \cdot 10^{6}$ & $\mathrm{~J} \mathrm{~m}^{-3} \mathrm{~K}^{-1}$ \\
Electron specific heat constant & $A_{e}$ & 98 & 71 & $\mathrm{~J} \mathrm{~m}^{-3} \mathrm{~K}^{-2}$ \\
Electron heat conductivity & $K_{e 0}$ & 401 & 318 & $\mathrm{~W} \mathrm{~m}^{-1} \mathrm{~K}^{-1}$ \\
Electron-phonon coupling factor & $g$ & $10 \cdot 10^{16}$ & $2.1 \cdot 10^{16}$ & $\mathrm{~W} \mathrm{~m}^{-3} \mathrm{~K}^{-1}$ \\
Reflection coefficient & $R$ & 0.6 & 0.64 & - \\
Absorption coefficient & $\alpha_{o p t}$ & $6.33 \cdot 10^{5}$ & $5.2 \cdot 10^{5}$ & $\mathrm{~cm}^{-1}$ \\
Density & $\rho$ & 8960 & $19.3 \cdot 10^{3}$ & $\mathrm{~kg} \mathrm{~m}^{-3}$ \\
Young's modulus & $E$ & 110 & 74 & $\mathrm{GPa}^{-}$ \\
Poisson number & $\nu$ & 0.35 & 0.43 & - \\
Surface tension & $\sigma$ & 1200 & 1000 & $\mathrm{mN} \mathrm{m}^{-1}$ \\
Donor layer thickness & $d$ & $50-200$ & $100-400$ & $\mathrm{~nm}^{-1}$ \\
Thermal diffusivity & $D$ & $1.1 \cdot 10^{-4}$ & - & $\mathrm{m}^{2} \mathrm{~s}^{-1}$ \\
Coefficient of thermal expansion & $\alpha_{t h}$ & $16.5 \cdot 10^{-6}$ & $14.2 \cdot 10^{-6}$ & $\mathrm{~K}^{-1}$ \\
\hline
\end{tabular}

Table 3.1: Material properties of copper and gold [86, 97].

simulations a time step of $20 \cdot 10^{-15} \mathrm{~s}$ is employed, which was found to be sufficiently small to describe the heating of a laser pulse in the picosecond regime. Next, to achieve a numerically stable solution and to limit the calculation time, the size of the individual mesh elements for the given time step was systematically varied within an interval from $10 \mathrm{pm}$ to $20 \mathrm{~nm}$. As a stability criteria, the lattice temperature of the donor layer at the location of the carrier-donor interface is calculated. Figure 3.5 indicates the variation of the calculated lattice temperature difference $\Delta T_{l}$ to the final temperature solution of the donor layer as a function of the inverse mesh element size $\Delta z$. It is obvious that exceeding a mesh element size of about $10 \mathrm{~nm}$ results in errors in the calculated temperature. Hence, the calculations presented in this work are based on a time step of $20 \cdot 10^{-15} \mathrm{~s}$ and a mesh element size of $1 \mathrm{~nm}$.

\subsubsection{Validity of the model}

To test the numerical validity of the model, the numerical results are compared against known specific analytical solutions. Figure 3.6 shows the calculated temporal evolution of the lattice (red solid line) and the electron temperature (dashed blue line) at the carrier-donor interface. The incident laser pulse $I_{0}(t)$ (green solid line) as described by equation (3.11) is plotted on the secondary axis. The laser pulse is centered at $t=2 \tau_{p}$ to ensure that the full energy of the laser pulse is considered in the simulation. The heating of the electrons follows directly the temporal evolution of the incident laser pulse. Accordingly, the heating of the electron subsystem starts at the same time as the laser pulse intensity start to 


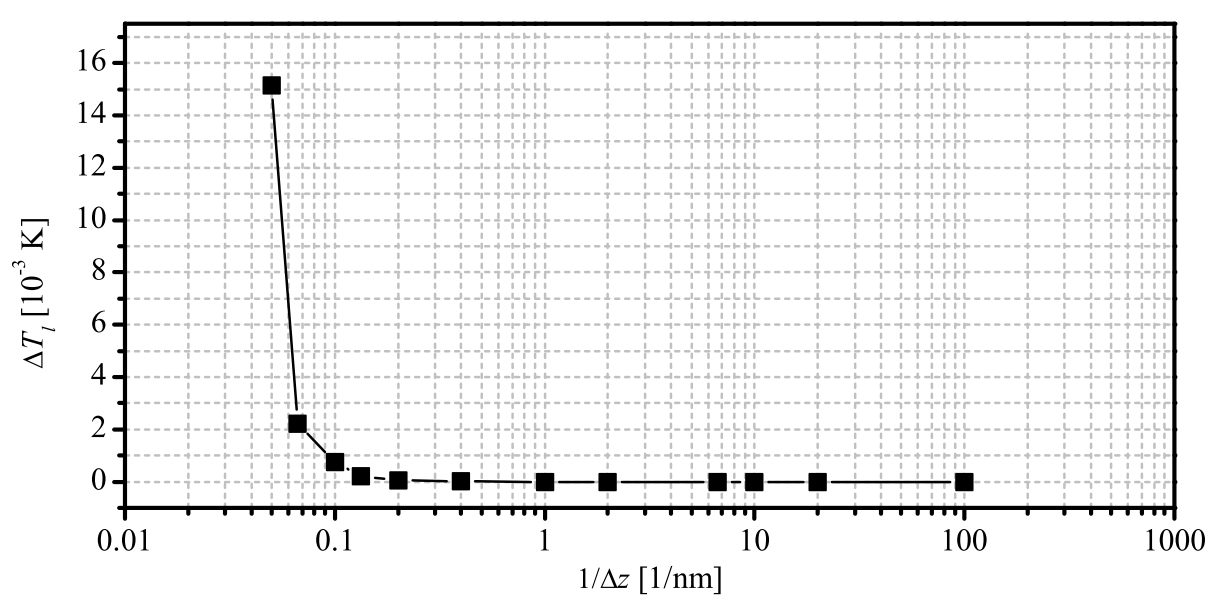

Figure 3.5: Temperature difference at the carrier-donor interface as a function of the inverse mesh-element size for a fixed time step size of $20 \cdot 10^{-15} \mathrm{~s}$. The simulations have been carried out using the material parameters of copper, and a laser peak fluence of $350 \mathrm{~mJ} / \mathrm{cm}^{2}$.

increase, i.e. at $t \approx 3$ ps. As the laser pulse reaches its maximum, the energy input from the laser pulse slows down, but is still dominant over the losses due to the coupling between the electrons and the lattice. Consequently, the electron temperature increases to its maximum shortly after. At this time instance, the lattice temperature readily exceeds the temperature of melting and increases further towards the equilibrium temperature. At approximately $30 \mathrm{ps}$ the electron and lattice temperatures reach equilibrium temperatures for the first time. Due to the nature of the TTM equations, the electron temperature undershoots the final equilibrium, which is reached at approximately $100 \mathrm{ps}$.

The numerical solution is validated through two key parameters, namely the conservation of energy and the computed lattice temperature with respect to a known analytic solution. First, the energy conservation is validated through an integral across the donor layer

$$
F_{v a l}=\frac{1}{1-R} \int_{0}^{d} C_{e}\left(T_{e}\right)+C_{l}\left(T_{l}\right) d z,
$$

which reflects the absorbed laser fluence $F_{v a l}$ in the full donor layer. The plot in figure 3.6 shows the time dependent evolution of such an integration for a incident laser peak pulse fluence of $350 \mathrm{~mJ} / \mathrm{cm}^{2}$. The value of the integral raises as the incident laser pulses is absorbed by the electrons. After about $25 \mathrm{ps}$, the full laser pulse energy is absorbed in the system, and regardless of the ongoing thermalization within the donor layer, the overall energy remains constant due to the applied boundary conditions. Secondly, the calculated temperature at $t=500 \mathrm{ps}$ is compared to the analytical solution of a heated film with similar dimensions. This is a valid approach, as in this time-scale the thermal penetration readily exceeds the donor layer thickness, and 


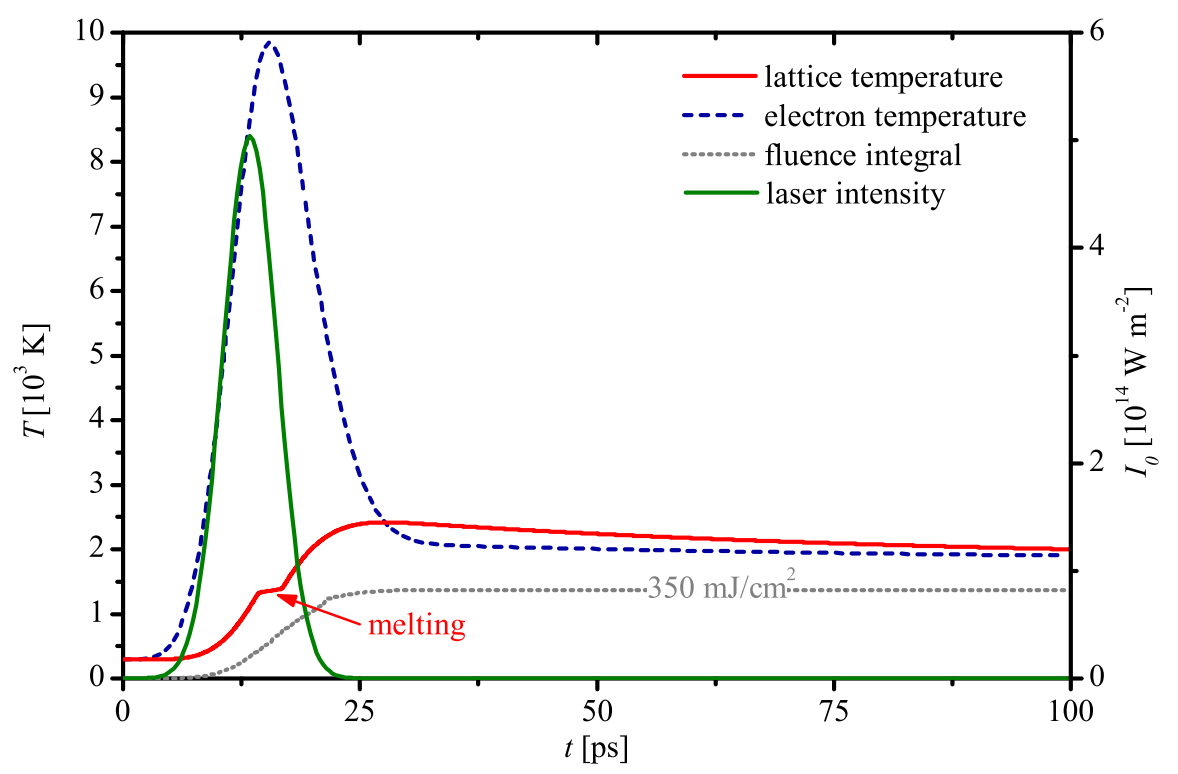

Figure 3.6: Lattice and electron temperature as a function of the time elapsed. The temperatures are calculated for a fixed laser peak fluence of $350 \mathrm{~mJ} / \mathrm{cm}^{2}$ at the carrier-donor interface, of a $200 \mathrm{~nm}$ copper donor layer. In addition, the incident laser pulse intensity and the recalculated laser fluence, that is used as one part of the model validation, are shown.

uniform heating of the film is achieved. Therefore, the temperature of a heated layer is estimated by equation (3.7) to be: $1863 \mathrm{~K}$. In comparison, the numerical model predicts a lattice temperature of $1816 \mathrm{~K}$. The temperature difference of $47 \mathrm{~K}(2.5 \%)$ is explained by the fact, that the analytical model does not consider the heat capacity of the electrons, and hence overestimates the resulting lattice temperature. 



\section{Chapter 4}

\section{Experimental methods}

In this chapter the experimental details of the LIFT setup and the laser sources used for the LIFT experiments are presented. Moreover, a detailed description of the sample preparation and the tools used for post-process analysis is provided.

\subsection{Introduction}

In the framework of this thesis, several aspects of the LIFT process are investigated experimentally. As a consequence, different experimental setups were designed in order to answer the relevant questions, formulated in section 1.3. For the sake of readability, the specific parts of each setup will be described in subsequent chapters. However, in this chapter the general LIFT setup, as it is used for all experiments, is described in detail. Next, the experimental methods used to determine the alignment of the donor layer with respect to the receiving substrate and the optical adjustment of the donor-carrier distance ("gap size") are discussed. Furthermore, the used laser sources for the LIFT, as well as the sample preparation and the tools used for the post-process analysis are summarized.

\section{$4.2 \quad$ Experimental setup}

\subsubsection{LIFT Setup}

Figure 4.1 shows a computer rendered isometric image of the setup, which was designed and build as a flexible tool to investigate the LIFT process. The setup consists of three major parts, namely: elements for donor positioning, elements for receiver positioning, and means for optical alignment of the receiver to the carrier. The donor is positioned in the $\mathrm{x} / \mathrm{y}$-plane using two ThorLABs PT1/M-Z8 translation stages, allowing to move the donor over a range of $25 \times 25 \mathrm{~mm}^{2}$. The 


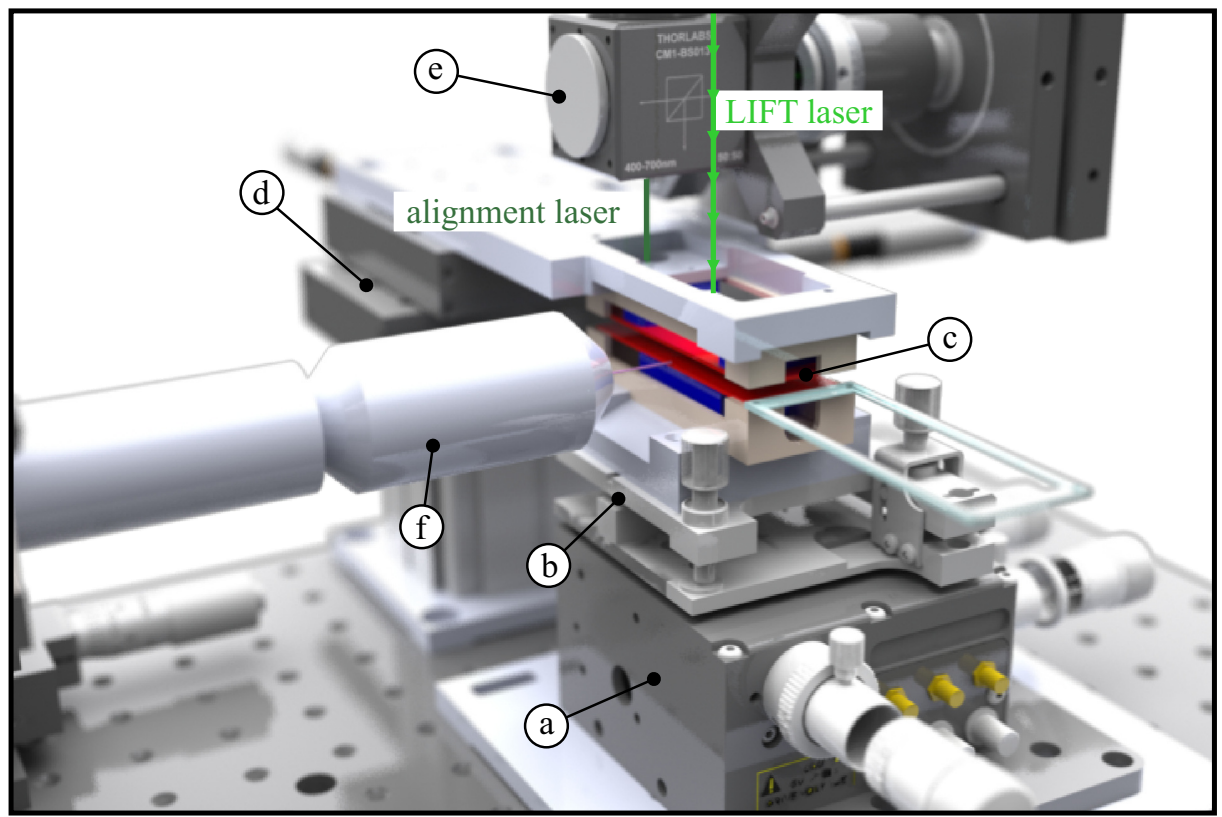

Figure 4.1: Computer rendered image of the LIFT setup as it is used for experiments. The design consists of: (a) a closed-loop 3-axis translation stage to adjust the separation between donor and receiver; (b) a pitch and roll tilt platform to adjust the alignment between donor and receiver. (c) The donor and the receiver substrates (red slides) kept in position by vacuum chucks (blue); (d) motorized translation stages used to position the donor in the x-, y-plane; (e) optical means to determine the angular alignment of the receiver with respect to the donor; (f) a microscope setup that is used to measure the gap size between the donor and the receiving substrate.

actual donor slide (top red slide) is mounted onto those stages, using rectangular air-bearings from LION PRECISION. Those air-bearings are made of an aluminum foam, which in combination with a vacuum source are used as flexible vacuum chucks. The gap size between the donor and the receiver is adjusted, by positioning the receiver substrate only. Therefore, a THORLABS MAX311D/M 3-axis translation stage is used. This stage offers micrometer accuracy positioning of the receiver, when used with the provided micrometer thumbscrew drives. However, for more accurate positioning, closed-loop piezo actuators are implemented in this stage, that allow for high accuracy positioning within a travel range of $20 \mu \mathrm{m}$. The angular orientation of the receiving substrate with respect to the donor is adjusted using a ThORLABS APR001/M pitch and roll tilt platform. To ensure, that the donor and the receiving substrate are aligned parallel with respect to each other, an optical detection system is employed. The gap size between the donor and the receiver is measured using a magnifying microscope, consisting of a 10× Mitutoyo Plan Apo Infinity-Corrected Long WD Objective in combination with a EDMUND OPTICS 


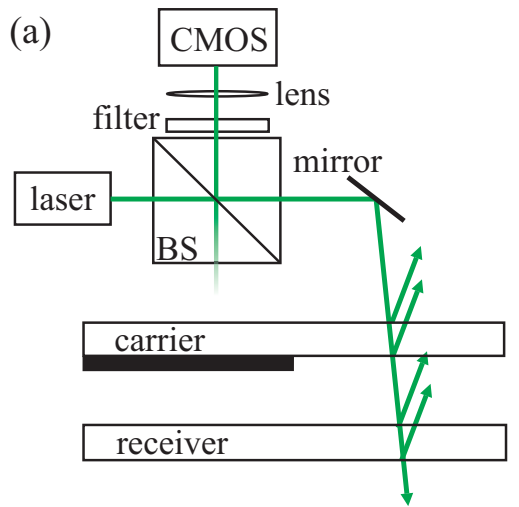

(b)

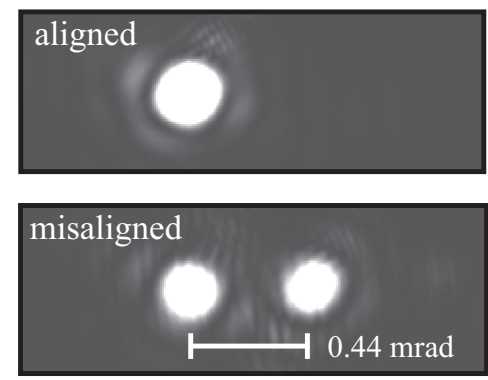

Figure 4.2: (a) Sketch of the optical system, which is used to measure the reflections of the incident laser beam on the each interface of the carrier and the receiver. (b) Shows examples of an aligned and misaligned receiver substrate.

InfiniTube FM System. The details of both, the alignment of the receiver with respect to the donor, as well as of the optical detection of the gap size are discussed in the next subsections.

\subsubsection{Receiver alignment}

To measure the alignment of the carrier and receiver with respect to each other, the reflections of a laser beam are exploited. To this end, a laser beam (THORLABS CPS532) is guided onto the carrier and aligned to normal incident with respect to the air-glass-interface of the carrier substrate, see figure 4.2. A CMOS camera (THORLABS DCC1545M) is used to detect the reflections of each interface as the beam propagates through the carrier and the receiver. Therefore, an achromatic doublet (THORLABS AC254-060-A) is used to focus the reflected beams of each interface onto the detector of the camera. Depending on the angular deviation of each reflection, the focus of each beam deviates from the zero order focus. In order to suppress in influence of ambient light, a bandpass-filter (ThORLABS FL532-10) is placed into beam path before the camera. The angular resolution that can be achieved by the custom made measurement device is given by the optical resolution (Rayleigh criterion) and the properties of the camera, such as pixel size. The resolvable displacement $\Delta x$ can therefore be estimated by

$$
\Delta x=1.22 \frac{\lambda f}{d_{A P}},
$$

where $\lambda$ relates to the wavelength of the laser diode and $f$ and $d_{A P}$ correspond to the focal length and the aperture/beam diameter of/before the lens, respectively. For the given setup $\left(f=60 \mathrm{~mm}, d_{A P}=1 \mathrm{~mm}, \lambda=532 \mathrm{~nm}\right)$ the minimal detectable separation $\Delta x$ is calculated to be $\approx 40 \mu \mathrm{m}$, corresponding to an angular resolution of $0.01 \mathrm{mrad}$. Here, the resolvable displacement exceeds the pixel size of the camera by one order 

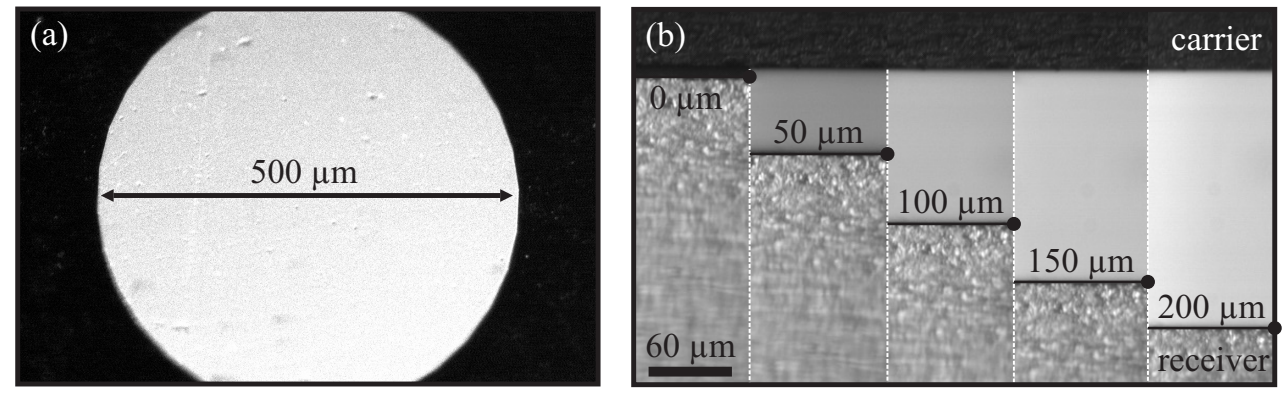

Figure 4.3: (a) Microscope image of a sphere with a known diameter of $500 \mu \mathrm{m}$, used as a test pattern in order to calibrate the microscope. (b) Microscope images of the carrier (donor layer is not visible) and the receiver at different gap sizes. The separation was set to values ranging from $0 \mu \mathrm{m}$ to $200 \mu \mathrm{m}$ using the micrometer thumbscrew of the translation stages.

of magnitude, which is sufficient to prevent anti-aliasing (Nyquist) and justifies to disregard the influence of the chip on the measurement.

\subsubsection{Measurement of the gap size}

To measure and set the gap size between the carrier and the receiver precisely, two methods were considered. First, the gap size could be controlled on the basis of the provided micrometer thumbscrew of the translation stages (figure 4.1 (a)). Therefore, the position of the carrier, when in contact with the receiver needs to be measured. The determination of this location was found challenging due to the varying properties of the materials that have been used as a receiving substrate. Finally, an optical inspection of the gap size, using a magnifying microscope setup was employed. To that end, a calibration of the microscope, i.e. the scaling factor $(\mu \mathrm{m} / \mathrm{pixel})$ is required. Here, a test pattern that consists of a circle with a known diameter of $500 \mu \mathrm{m}$ is used, see figure 4.3 (a). Through this approach a scaling factor of $0.2 \mu \mathrm{m} /$ pixel is obtained. Next, the accuracy of the receiver placement, i.e. the control of the gap size based on the accuracy of the micrometer thumbscrew was investigated. Figure 4.3 (b) shows the carrier and the receiver, with different preset gap sizes ranging from $0 \mu \mathrm{m}$ to $200 \mu \mathrm{m}$. These gap sizes were set purely relying on the accuracy of the micrometer thumbscrews of the translation stage. Though, equidistant steps were intended, the measured distance shows a significant variation in the actual measured gap size using the optical microscope. The resulting standard deviation is measured to be $10.9 \mu \mathrm{m}$. For this reason the first approach, which relies on the accuracy of the thumbscrews, is discarded and the gap size is controlled with the optical microscope at any time, to ensure a proper positioning of the receiving substrate. 


\begin{tabular}{lll} 
Parameter & Trumpf TruMicro 5050 & Litron Lasers NANO S \\
$M^{2}$ & $<1.3$ & - \\
Pulse duration & $6.7 \mathrm{ps}$ & $\sim 6-8 \mathrm{~ns}$ \\
Beam waist & $3 \mathrm{~mm}$ & $4 \mathrm{~mm}$ \\
Beam Divergence & $0.142 \mathrm{mrad}$ & $\sim 2.5 \mathrm{mrad}$ \\
Central wavelength & $1030 \mathrm{~nm}$ & $532 \mathrm{~nm}$ \\
Repetition rate & $400 \mathrm{kHz}$ & $15 \mathrm{~Hz}$ \\
Max. pulse energy & $250 \mu \mathrm{J}$ & $65 \mathrm{~mJ}$ \\
Polarization & linear & linear \\
\hline
\end{tabular}

Table 4.1: Overview of the properties of the laser sources used for the LIFT process.

\subsubsection{Laser beam delivery}

Two laser sources with pulse durations in the picosecond and the nanosecond regime were employed for the investigation on the governing physics of the LIFT process, see table 4.1. The fundamental wavelength of these sources are $1030 \mathrm{~nm}$ (Yb:YAG) and $1064 \mathrm{~nm}$ (Nd:YAG), which were converted by employing second harmonic generation units, referred as SHG. The resulting central wavelength of $515 \mathrm{~nm}$ and $532 \mathrm{~nm}$ are used for the LIFT experiments. The laser power was adjusted by the combination of a $\lambda / 2$ wave-plate and a polarizing beam splitter (PBS). The long term power variation was measured to be $\pm 2 \%$. Manipulation of the laser beam was achieved by the use of a two-mirror Galvo-scanner (SCANLAB intelliSCAN 14). To maintain a constant focus position across the surface of the carrier-donor interface, a telecentric $\mathrm{f}$ - $\theta$-ronar scan lens (LINOS) with a focal length of $100 \mathrm{~mm}$ was employed. The absolute positioning accuracy, given by the encoder of the Galvo-scanner, is specified to be $1 \mu \mathrm{m}$. Unless stated differently, the LIFT experiments were carried out at constant temperature and humidity, as specified by the clean-room norm ISO 7 .

\subsubsection{Beam waist}

The beam waist $\omega_{0}$ of the focused laser beam, as well as its error is determined experimentally for the experimental LIFT setup. To that end, the well-known $D^{2}$ method [98] was employed. The lateral variation of the characterized ejection crater is provided as

$$
D_{\text {mod }}^{2}=2 \omega_{0}^{2} \ln \left(\frac{F}{F_{\text {mod }}}\right)
$$

with $D_{\text {mod }}$ being the ejection crater diameter in the donor and $F_{\text {mod }}$ being the modification threshold fluence. Those fluence scans were performed in LIFT configuration. The variation of the squared crater diameter with respect to the laser fluence is shown in figure 4.4. As can be observed in this figure, several fluence scans involving different materials, as well as different layer thicknesses have been employed. For each fluence scan, a new donor layer was used, and the stages that are used to control the position of the donor layer were moved to an arbitrary chosen 


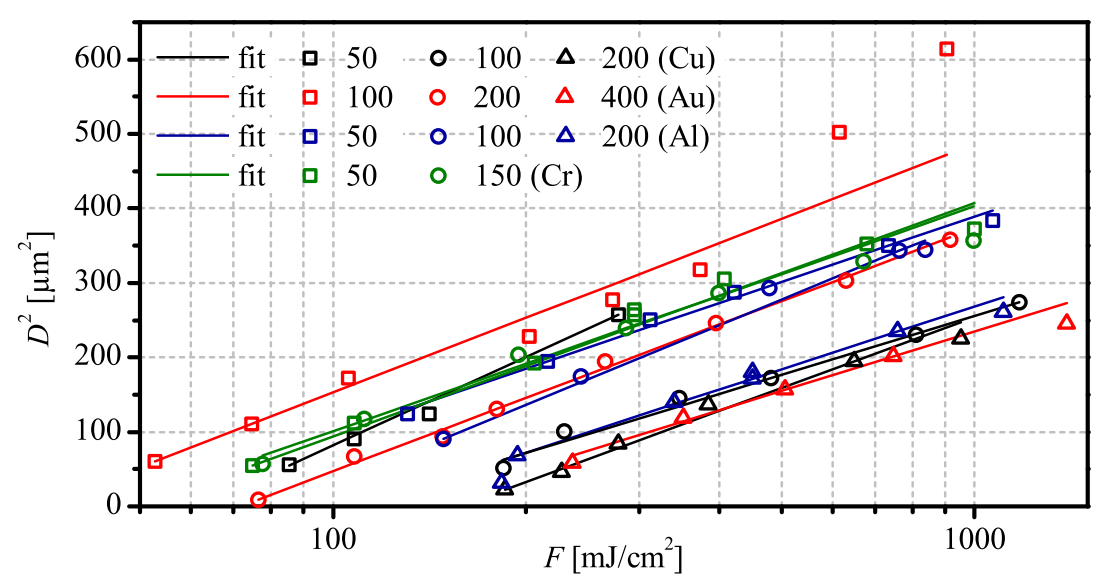

Figure 4.4: Squared diameter $D^{2}$ of the ejection crater after LIFT in copper, gold, aluminum, and chromium donor layers as a function of the laser fluence. The solid lines represent the least-squares-fit based on equation (4.2).

new location. Finally, the beam waist of the focused laser beam was determined from the averaged slope of those measurements. To this end, the obtained error represents a realistic estimate of the actual error as it reflects several experimental effects related to the replacement of the samples as well as the positioning of the donor stages. Here, the actual beam waist is determined to be $8.3 \pm 0.6 \mu \mathrm{m}$ and is considered for the experimental results presented in chapter 5 .

\subsection{Materials and sample preparation}

As already was described, the LIFT process involves two substrates referred as donor layer and receiver. Varying the physical properties (grain size, purity, adhesion, roughness, contamination) or the chemical composition [41] of these substrates may lead to uncontrolled fluctuations in the ejection process or lead to undesired conditions during droplet impact on the receiver. Therefore, the preparation of the donor layer and the receiver needed special care. As a carrier substrate $76 \times 26 \times 1 \mathrm{~mm}$ extra-white glass slides (MENZEL GLÄsER AG00000102E) were used. Donor layers of various sizes of gold or copper were deposited on such glass substrates using magnetron sputtering (sputter rate: $23 \mathrm{~nm} / \mathrm{min}$ ). The resulting layer thickness was found to exhibit a variation of $\pm 10 \%$. Prior to the deposition of the donor material, the substrates were first ultrasonically cleaned using isopropanol, ethanol and acetone. Next, the substrates were washed in a bath of $99 \%$ nitric acid for ten minutes, followed by a quick dump rinse. Prior to sputtering, the slides were baked at $120{ }^{\circ} \mathrm{C}$ for ten minutes to evaporate any remaining water. Though, absorption of the incident laser pulse within the carrier is negligible, the carrier may be affected by the heated donor, which subsequently 


\begin{tabular}{llll} 
Compound & Chemical & Proportion [\%] & $\begin{array}{l}\text { Boiling } \\
\text { temperature }[K]\end{array}$ \\
\hline Silicon Dioxide & $\mathrm{SiO}_{2}$ & 72.20 & 2500 \\
Sodium Oxide & $\mathrm{Na}_{2} \mathrm{O}$ & 14.30 & 2223 \\
Potassium Oxide & $\mathrm{K}_{2} \mathrm{O}$ & 1.20 & decomposes at 623 \\
Calcium Oxide & $\mathrm{CaO}$ & 6.40 & 3123 \\
Magnesium Oxide & $\mathrm{MgO}$ & 4.30 & 3870 \\
Aluminum Oxide & $\mathrm{Al}_{2} \mathrm{O}_{3}$ & 1.20 & 3250 \\
Ferric Oxide & $\mathrm{Fe}_{2} \mathrm{O}_{3}$ & 0.03 & decomposes at 1839 \\
Sulfur Trioxide & $\mathrm{SO}_{3}$ & 0.30 & 318 \\
\hline
\end{tabular}

Table 4.2: Chemical composition of extra-white glass [99].

heats the carrier through normal heat conduction. Hence, the chemical composition of the carrier may be of relevance and is detailed in table 4.2. The receiver is a standard $76 \times 26 \times 1 \mathrm{~mm}$ microscope slide on which a $100 \mathrm{~nm}$ to $400 \mathrm{~nm}$ thick coating of gold or copper was deposited using the same sputter technique used to create the donor layer. These coated receivers were used to maximize the adhesion between the deposits and the receiver in case of solid phase transfer, and to optimize the heat conduction of an impacting liquid droplet on a receiver, as will be discussed in detail in chapter 8 .

\subsection{Post-process analysis}

To obtain optical images as well as 3D data of the processed donor layers and the deposits on the receiver, a laser scanning confocal microscope (KEYENCE VK 9700) was used. For a detailed analysis of the donor layer (see chapters 6 and 7), scanning electron microscope (SEM) images were acquired, using a ZeISS Merlin Scanning Electron Microscope (HR FEG SEM). Cross-sections, as presented in chapters 7 and 8 were obtained using a focused ion beam (FIB) machine (FEI NOVA 600 Dual Focused Ion Beam machine). The force measurements as discussed in chapter 8 , were obtained with the Nanosurf Easyscan Atomic Force Microscope (AFM). 



\section{Chapter 5}

\section{Ejection regimes in picosecond LIFT}

In this chapter* the impact of the applied laser fluences on the ejection process during LIFT is discussed. Different ejection regimes are observed and interpreted on the basis of a two temperature model. The governing physics are assessed and validated using energy balances.

\subsection{Introduction}

As outlined in chapter 1 , the LIFT process is a $3 \mathrm{D}$ direct-write method with a high industrial potential for precision printing of various materials, which is in particular interesting for printing of high melting point metals. In order to understand the ejection mechanism during LIFT and thereby improve the quality of the deposition, time-resolved imaging experiments are performed. In this chapter, visualizations of ejection events at high spatial (sub- $\mu \mathrm{m})$ and temporal resolutions, of picosecond LIFT of copper, as well as of thin gold films with a layer thickness of $50 \mathrm{~nm} \leq d \leq 400 \mathrm{~nm}$ are presented. Based on those images, different types of ejections and the corresponding ejection velocities occurring during LIFT of copper and gold are identified. To interpret the experimental results the two-temperature model introduced in section 3.3 is employed, providing key evidence for the underlying ejection mechanism. Based on this evidence, the energy balances required to model the ejection regimes and ejection velocities are proposed. In addition, the fluence threshold below which no ejection is observed is quantitatively described using a balance between the surface energy of the locally melted film and

*Parts of this chapter have been published in Physical Review Applied, 3:024001, 2015, Ralph Pohl, Claas Willem Visser, Gert-Willem Römer, Detlef Lohse, Chao Sun, and Bert Huis in 't Veld. Ejection Regimes in Picosecond Laser-Induced Forward Transfer of Metals. 


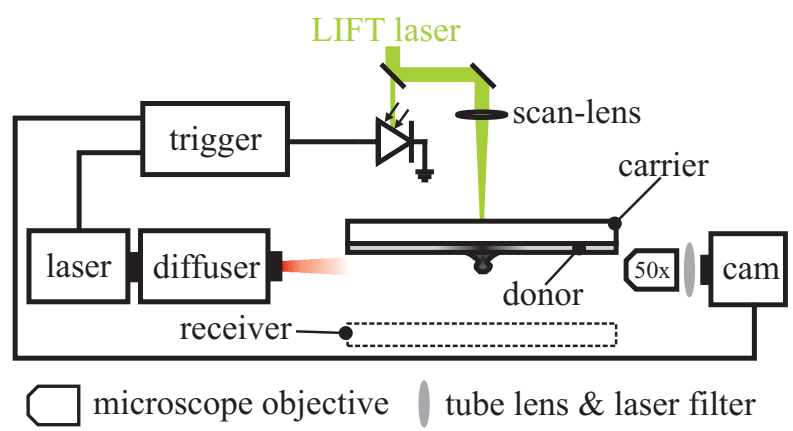

Figure 5.1: Sketch of the experimental setup used for picosecond LIFT. The experiments were conducted without a receiving substrate. LIFT laser beam in green. Illumination laser beam in red.

its inertia. This chapter is structured as follows. First, details of the experimental methods with a focus on the imaging setup are briefly explained in section 5.2. Next, in section 5.3 high-resolution images of the ejection dynamics are presented and discussed with respect to the observed ejection regimes. To this aim, the experimental results are interpreted using the two-temperature model detailed in section 3.3, which provides the key parameter settings for each regime, and provides a simple estimate of the ejection fluence threshold. Finally, the implications and limitations of these results are discussed in section 5.4, followed by the conclusions in section 5.5.

\subsection{Methods}

The experiments are performed using the setup schematically shown in figure 5.1. For LIFT, a Yb:YAG laser source with a fixed pulse duration of $6.7 \mathrm{ps}$, a wavelength of $515 \mathrm{~nm}$ (SHG) and a Gaussian beam profile with a beam quality factor of $M^{2} \leq 1.3$ was used. The beam was focused onto the carrier-donor interface using a f-thetaronar scan-lens with a focal length of $100 \mathrm{~mm}$. The beam waist $\left(1 / \mathrm{e}^{2}\right)$ was measured to be $8.3 \pm 0.6 \mu \mathrm{m}$. The fluence values in the following represent peak fluences. The maximum error of measurement in the fluence is $20 \mathrm{~mJ} / \mathrm{cm}^{2}$, as was determined using the $\mathrm{D}^{2}$-Method [98], see section 4.2.5. Copper and gold films with thicknesses $50 \mathrm{~nm}<d<400 \mathrm{~nm}$ (magnetron sputtered at $23 \mathrm{~nm} / \mathrm{min}$ onto a $1 \mathrm{~mm}$ thick glass carrier) are used as donor layer. To optimize the experimental imaging conditions, the receiving substrate was omitted. Further details of the LIFT setup and the preparation of the donor layers are provided in chapter 4.

Side view images were recorded using a dual-shot CCD camera (PCO Sensicam), mounted to a microscope with a $50 \times$ long-distance objective (Mitutoyo Plan Apo \#46-146), see figure 5.1. Brightfield flash illumination was provided by a dual-cavity Nd:YAG laser with a pulse duration of $6 \mathrm{~ns}$. A LAVISION high-efficiency diffuser was used to diffuse this laser pulse and thereby prevent fringes in the images. To 


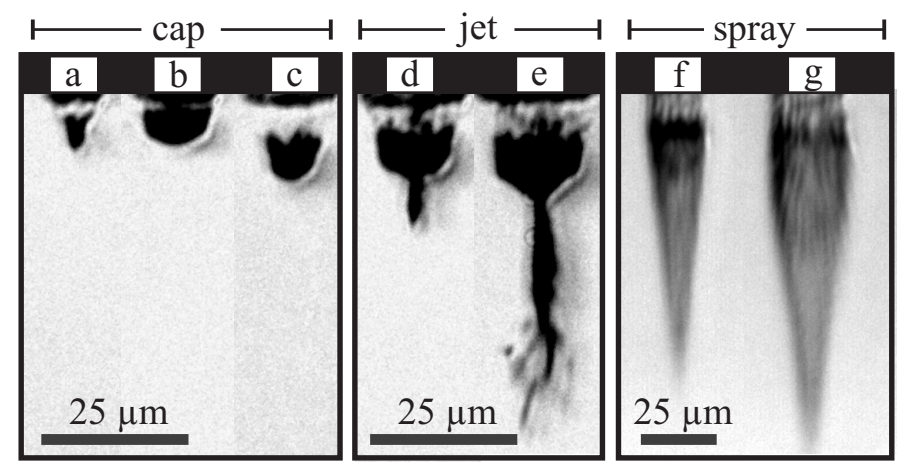

Figure 5.2: Ejection of a $200 \mathrm{~nm}$ copper film, visualized $125 \mathrm{~ns}$ after the incident laser pulse. Figure (a) to (g) correspond to increasing fluence values of 314, 392, 480, 660, 864, 1060 , and $1576 \mathrm{~mJ} / \mathrm{cm}^{2}$, respectively. The black bar (not to scale) at the top of the images schematically shows the location of the donor layer. Images (a) to (c) illustrate the cap ejection regime observed at low fluence levels; (d) and (e) show the formation of a jet from the apex of the cap at intermediate fluence levels, and (f) and (g) show the ejection of a fast copper spray. In image (g), some droplets are visible as lines, as their high velocity results in strong motion blur, as the tip speed is $700 \mathrm{~m} / \mathrm{s}$.

determine the ejection velocity of the ejected donor, the vertical distance between the ejection crater in the donor layer and the tip of the ejection was measured and divided by the time between the ejection event and the frame illumination event. For triggering, a pulse delay-generator was used (BERKELEY Nucleonics BNC 575). The trigger sequence was started by the output of a photo diode exposed to the beam path of the LIFT laser. All components were selected to achieve a high temporal resolution, resulting in a temporal measurement error of less than $10 \mathrm{~ns}$. The spatial resolution of the imaging system is either limited by the specified diffraction limit of $0.5 \mu \mathrm{m}$ or by motion blur for ejection velocities exceeding $100 \mathrm{~m} / \mathrm{s}$.

\subsection{Results}

\subsubsection{Ejection regime classification}

First, the ejection characteristics at different fluence levels are studied and described in a qualitative manner. Figure 5.2 shows the fluence-resolved ejection dynamics for a $200 \mathrm{~nm}$ copper film. Figures 5.2 (a) to 5.2 (c) show typical ejections in the low-fluence regime $\left(F \leq 600 \mathrm{~mJ} / \mathrm{cm}^{2}\right)$, where the ejection of a cap is observed. For intermediate fluences $\left(600 \mathrm{~mJ} / \mathrm{cm}^{2} \leq F \leq 740 \mathrm{~mJ} / \mathrm{cm}^{2}\right)$, a jet-like feature is formed on the apex of the ejected cap, see figure 5.2 (d) and 5.2 (e). At even higher fluence levels $\left(F \geq 740 \mathrm{~mJ} / \mathrm{cm}^{2}\right)$, ejection of a spray is observed, see Figure 5.2 (f) and $5.2(\mathrm{~g})$. These sprays are characterized by a cloud of particles or droplets, instead of the more coherent features as observed in the cap ejection regime. The sometimes spectacular dynamics of each regime[100] are discussed in more detail in chapter 6 . 


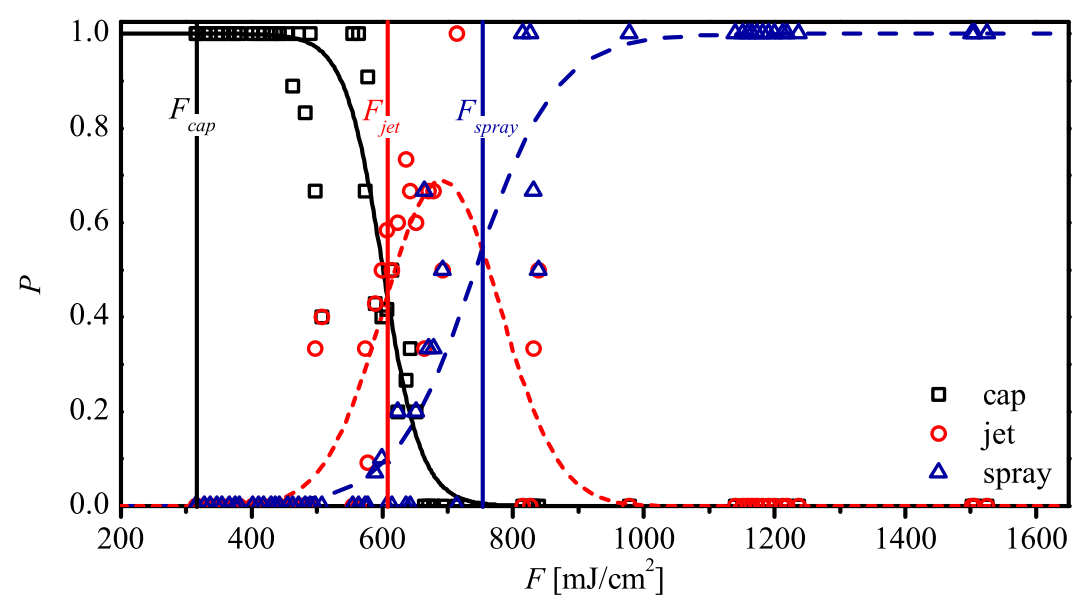

Figure 5.3: Probability $P$ of the type of ejections observed (cap, jet or spray) versus the incident laser fluence.

Establishing the threshold fluence levels between these regimes proved challenging, as experiments with identical parameter settings sometimes resulted in different regimes, in particular close to the transition fluence levels. Therefore numerous experiments were performed and the incidence rate of each regime was binned as a function of the laser fluence. For the $200 \mathrm{~nm}$ copper film, 388 ejection events were categorized and binned using a bin width of $10 \mathrm{~mJ} / \mathrm{cm}^{2}$. Figure 5.3 shows the probability of each ejection regime for each bin. The lower fluence thresholds for the cap-, jet- and spray-regimes were based on a regime incidence rate of $50 \%$, yielding values of 320,600 , and $740 \mathrm{~mJ} / \mathrm{cm}^{2}$, respectively. These thresholds are related to the film temperature, providing evidence for the ejection driving mechanism as to be discussed in the following.

\subsubsection{Temperature analysis}

For further discussion, the observed ejection regimes are interpreted on the basis of the two-temperature model, detailed in section 3.3. The temporal evolution and spatial distribution of the electron- and lattice temperatures are computed for two prominent locations in the donor layer, namely the carrier-donor interface and the donor-air interface. A typical simulation result of copper is plotted in figure 5.4, showing the interface lattice temperatures and the temperature difference between the electron and the lattice temperature $\Delta T_{e-l}$ as a function of time. First, the laser pulse is absorbed by the electron subsystem in the optical absorption depth, which has a thickness of $1 / \alpha_{\text {opt }} \approx 15 \mathrm{~nm} \ll d$, resulting in electron temperatures up to almost $10^{4} \mathrm{~K}$ at the carrier-donor interface (blue triangles in figure 5.4). Subsequently, the lattice is heated by the electrons and reaches a peak temperature of $\approx 2600 \mathrm{~K}$ on a timescale of the relaxation time of the electron-phonon system $\left(\tau_{e p} \approx 20 \mathrm{ps}\right)$. 


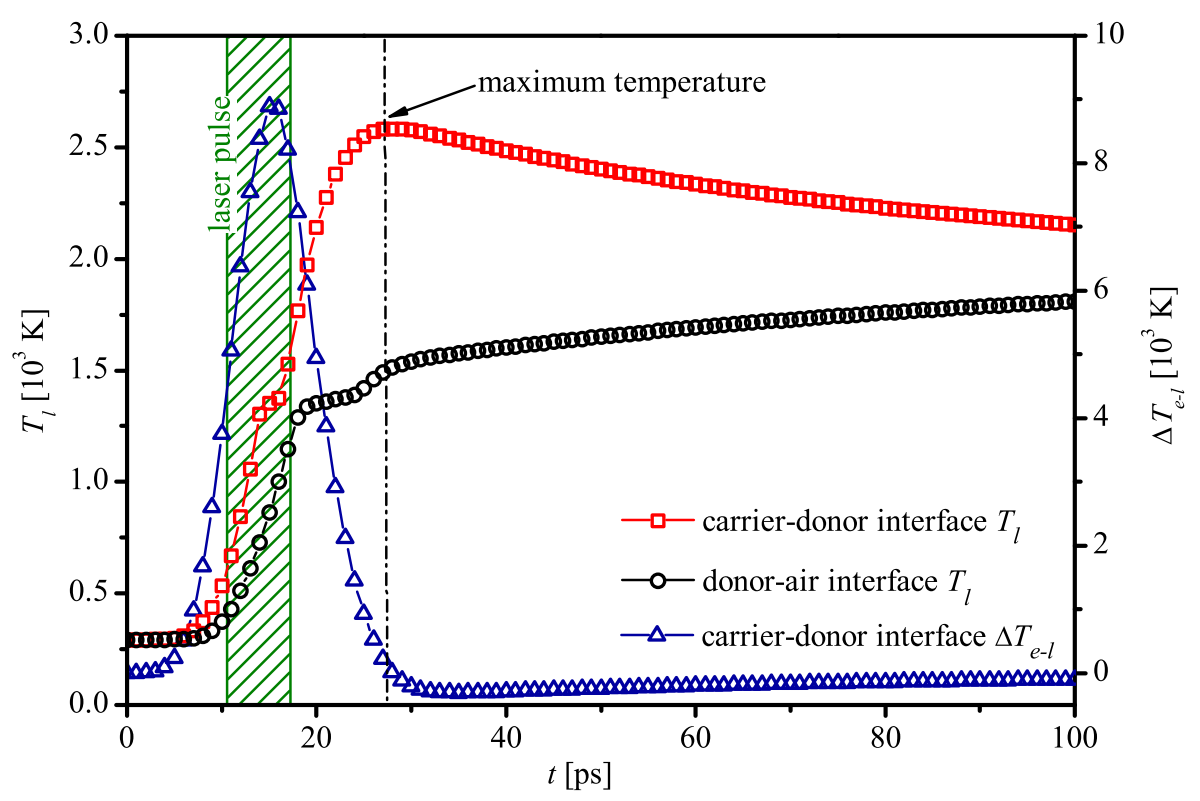

Figure 5.4: Calculated lattice temperature of copper $T_{l}$ at the carrier-donor interface (squares, left axis) and the donor-air interface (circles, left axis) as a function of time (at $t=0$ the simulation starts; the peak pulse energy is reached at $t=2 \tau_{p}=13.4 \mathrm{ps}$ ), for $F=376 \mathrm{~mJ} / \mathrm{cm}^{2}$. The hashed area indicates the full width half maximum of the laser pulse duration. The difference between the lattice and the electron temperature at the carrierdonor interface (triangles, right axis) illustrates the high electron temperatures reached and the electron cooling on the electron-phonon relaxation timescale $(\approx 20 \mathrm{ps})$.

Ultimately, an almost homogeneous temperature is reached in the donor layer after $t \approx 100 \mathrm{ps}$, corresponding to the thermal diffusion timescale $\tau_{D}=d^{2} /(2 D) \approx 180 \mathrm{ps}$, with $D=1.1 \cdot 10^{-4} \mathrm{~m}^{2} / \mathrm{s}$ the thermal diffusivity.

Next, the physical conditions in the donor layer during the process are related to the observed ejection regimes. Figure 5.5 shows the calculated maximum interface temperatures of the copper film as a function of the laser fluence. The temperature plateaus at $T_{l} \approx 1400 \mathrm{~K}$ and $T_{l} \approx 2800 \mathrm{~K}$ indicate the melting- and evaporation phase changes, respectively. Here, increasing the fluence levels only results in the phase change of a larger material fraction. The onset of melting, i.e. partial melting of copper close to the carrier-donor interface, is predicted to occur at $F \approx 120 \mathrm{~mJ} / \mathrm{cm}^{2}$. At a fluence of $F \approx 350 \mathrm{~mJ} / \mathrm{cm}^{2}$ the copper film is completely melted. The onset of evaporation, i.e. partial evaporation of copper close to the carrier-donor interface, occurs at $F \approx 400 \mathrm{~mJ} / \mathrm{cm}^{2}$. For fluences $\geq 610 \mathrm{~mJ} / \mathrm{cm}^{2}$, the full layer has reached the steady-state vaporization temperature and increasing the fluence only results in a larger vaporized fraction of the film. A further temperature increase is expected only for fluences resulting in complete film vaporization, which are beyond the current parameter space. 


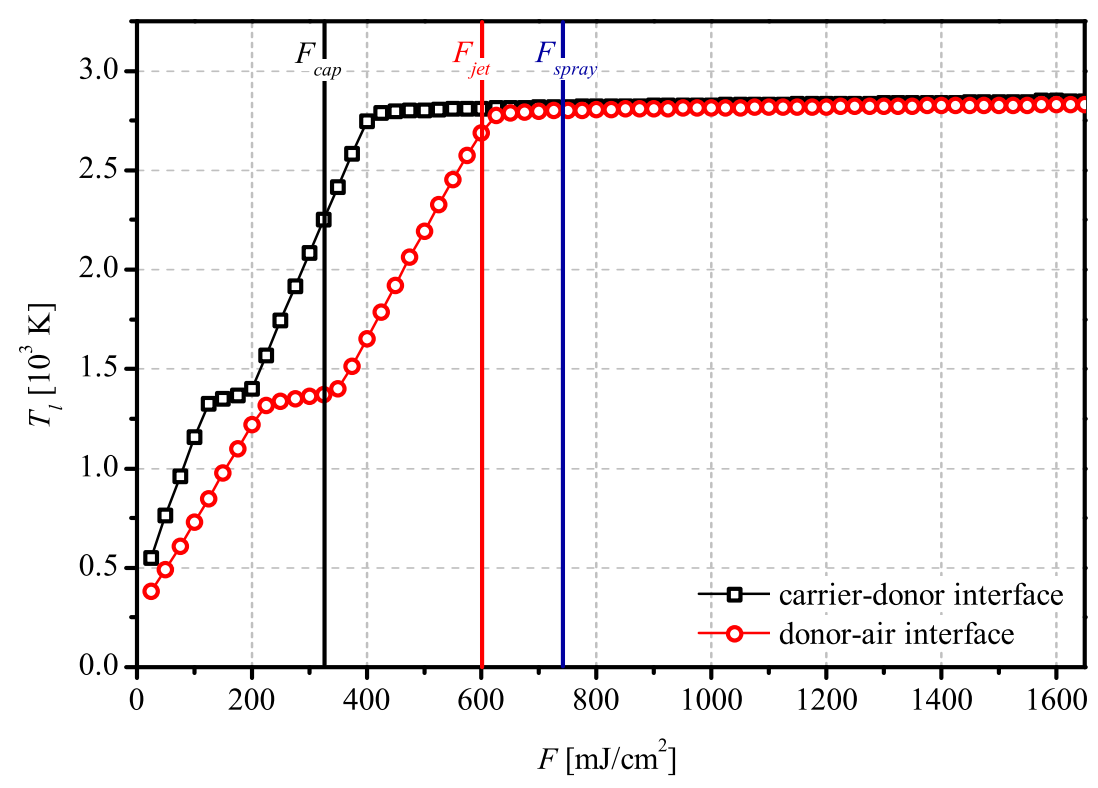

Figure 5.5: Computed maximum temperature as function of laser fluence, at the interfaces of a $200 \mathrm{~nm}$ copper film.

The transition fluence levels extracted from figure 5.3, separating the ejection regimes, are re-plotted in figure 5.5 (vertical lines). The ejection threshold for cap ejection $F_{c a p}=320 \mathrm{~mJ} / \mathrm{cm}^{2}$ coincides with the full melting of the donor film. The transition to the jet ejection regime occurs when the full layer reaches the evaporation temperature. Therefore in the following discussions it is assumed that the driving mechanism of the jet and the spray are identical. This quantitative correspondence between the phase changes and the threshold fluences for cap and jet ejection suggests the following ejection mechanisms:

- cap ejection by thermally-induced stress relaxation,

- threshold ejection fluence governed by the capillary-inertial energy balance,

- jet- and spray ejection by partial film vaporization,

which will be discussed each in the next section.

\subsubsection{Ejection mechanisms}

\section{Cap ejection by thermally-induced stress relaxation}

As shown in figure 5.5, the model does not predict any vaporization of the copper donor layer for fluence levels just above the cap ejection fluence threshold. Therefore, thermal compression of the metal film and subsequent release of elastic energy is 


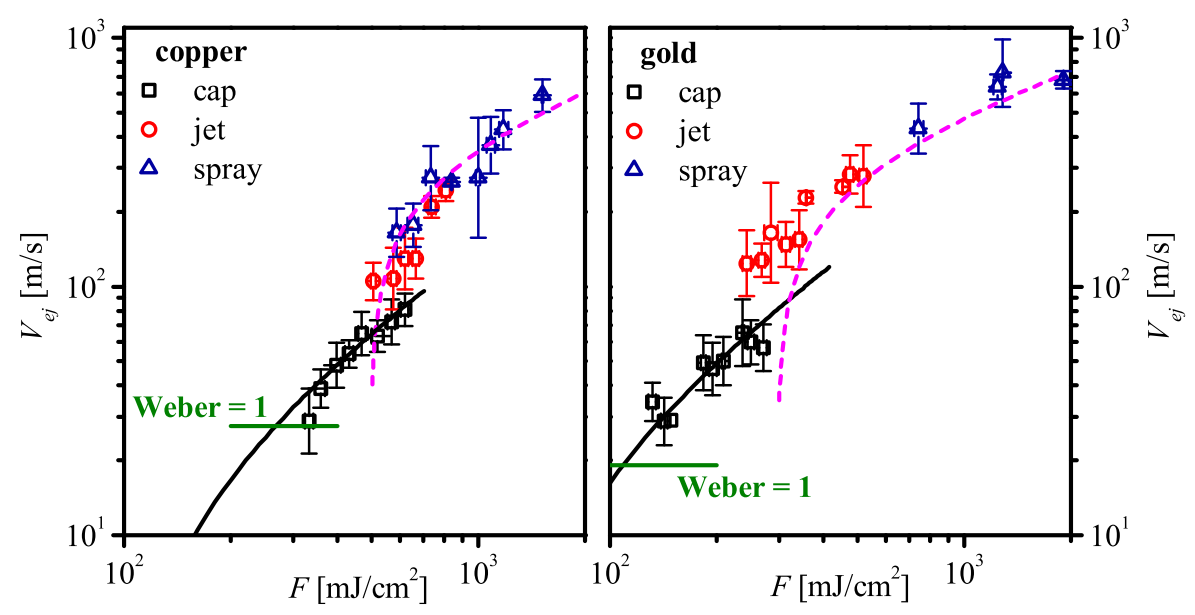

Figure 5.6: Ejection velocity as a function of the laser fluence, for a $200 \mathrm{~nm}$ copper film (a) and a $160 \mathrm{~nm}$ gold film (b). The different markers indicate measurements of velocities of the three ejection regimes analogous to figure 5.2. The green horizontal line indicates the predicted minimum velocity; the curved black solid lines show the modeled cap ejection velocity i.e. equation (5.5), and the curved dashed purple line indicates the spray- and jet ejection velocity model, i.e. equation (5.9). The data points represent grouped measurements with error bars indicating the standard deviation, each data point consists of at least 10 individual measurements.

proposed as the driving mechanism as proposed by Veiko et al. [36] and by Ivanov et al. [40]. To further study the characteristics of this driving mechanism, the elastic energy $E_{E}$ in the donor layer is modeled as

$$
E_{E}=\int_{0}^{d} A_{s r f} K u^{2} d z \approx \frac{1}{2} d A_{s r f} K \alpha_{t h}^{2} \Delta T^{2},
$$

using thermal expansion $u=\alpha_{t h} \Delta T$ with $\alpha_{t h}$ the thermal expansion coefficient of the material and $\Delta T$ the lattice temperature increase due to the absorbed laser energy, a bulk modulus of $K=\frac{E}{3(1-2 \nu)}$ with Young's modulus $E$ and the Poisson ratio $\nu$, and a surface area $A_{s r f}$. For a laser pulse resulting in homogeneous heating of the complete film thickness, this yields

$$
E_{E}=\frac{1}{2} d A_{s r f} K \alpha_{t h}^{2} \Delta T^{2}
$$

in which the temperature increase $\Delta T$ is modeled as

$$
\Delta T=\frac{(1-R) F-d L_{m}}{d C_{l}},
$$

with $L_{m}$ the melting enthalpy, $R$ the reflection coefficient of the material, and $C_{l}$ its lattice heat capacity. It is assumed that all elastic energy is subsequently converted 
into kinetic energy $E_{k i n}$ of the ejected cap. Then by equating the elastic energy to the kinetic energy of an ejected cap

$$
E_{k i n}=\frac{1}{2} \rho d A_{s r f} V_{e j}^{2},
$$

where $\rho$ is the density, the ejection velocity $V_{e j}$ in the elastic regime is derived as:

$$
V_{e j}=\alpha_{t h} C_{0} \Delta T \sqrt{K / \rho},
$$

with $C_{0}$ a fitting factor. Figure 5.6 shows these calculated velocities based on the calculated temperature increase $\Delta T$ and are compared to measured values of the velocities in the cap ejection regime. In this figure, the solid lines represent the calculated velocities for copper (with $C_{0}=0.35$ ) and for gold (with $C_{0}=0.7$ ). As can be observed, using fitting factors of order one, good agreement between the model and the measurements is obtained.

\section{Threshold ejection fluence governed by the capillary-inertial energy balance}

Surprisingly, only cap ejections with velocities exceeding $\approx 20 \mathrm{~m} / \mathrm{s}$ are observed. This is the case, even at fluence levels just above the ejection threshold, as shown in figure 5.6. Previous reports by Brown et al. [24] have shown that for sub-ejection threshold fluence levels the film is accelerated, but retracted by surface tension before it can escape the liquid donor layer. This suggests that ejection only takes place if the kinetic energy of the ejected material exceeds its surface energy. Dividing the kinetic energy $E_{k i n}$ by the surface energy $E_{s}$ yields

$$
\frac{E_{k i n}}{E_{s}} \approx \frac{\rho d V_{e j}^{2}}{\sigma}=\mathrm{We}_{e j},
$$

with $V_{e j}$ the maximum ejection velocity, $\sigma$ the surface tension of molten copper, and $\mathrm{We}_{e j}$ the Weber number, i.e. the ratio of inertial energy and surface energy. Using a threshold Weber number of $\mathrm{We}_{e j}=1$, the minimal obtainable ejection velocity is readily determined from equation (5.6). As shown in figure 5.6, this velocity matches the measured minimum velocities reasonably well.

Also, using the condition of $\mathrm{We}_{e j}=1$, the minimum cap ejection fluence $F_{c a p}$ is readily determined. Combining equations (5.3), (5.5), (5.6), and solving the resulting equation for the fluence yields

$$
F_{\text {cap }}=\frac{1}{1-R}\left(\frac{d C_{l}}{\alpha_{t h} C_{0}} \sqrt{\frac{\sigma}{K d}}+d L_{m}\right),
$$

where again for gold $C_{0}=0.7$ and for copper $C_{0}=0.35$ should be used. This ejection threshold fluence $F_{c a p}$ is plotted as a function of the film thickness in figure 5.7, showing good agreement to the measured data for copper and gold films of various thicknesses. This suggests that the condition $\mathrm{We}_{e j}=1$ provides a simple but yet robust criterion for determination of the threshold fluence for LIFT of locally liquid metal films. 


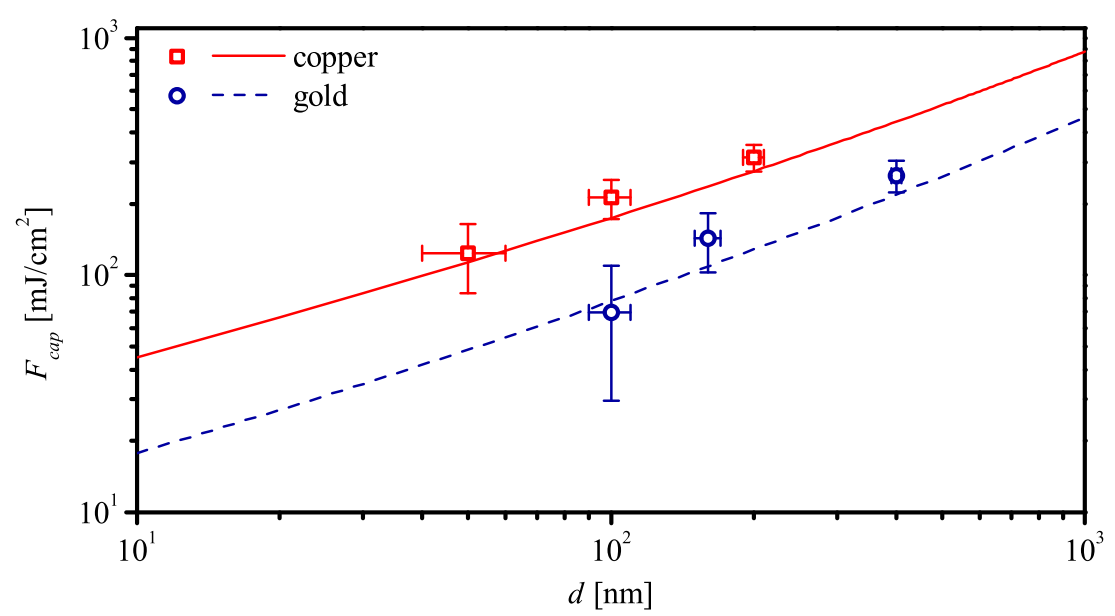

Figure 5.7: Threshold ejection fluence $F_{c a p}$ as a function of the film thickness, for copper and gold films. The markers indicate measured values, the lines indicate the prediction according to equation (5.7).

\section{Jet- and spray ejection by partial film vaporization}

For both jet- and spray ejection events, vaporization is predicted by the TTM to occur within the whole film, see figure 5.5. Therefore, vapor-driven ejection is assumed for these regimes. The shape of the ejections in these regimes, as can be observed from figure 5.2 are likely due to the Gaussian spatial power density distribution of the focused laser pulse. That is, in the jetting regime vaporization only occurs in a small spot in the center of the melt area, whereas in the spray ejection regime this occurs in almost the full molten area. The similarity of the jet- and spray regimes is confirmed by the partial overlap, in terms of fluence levels, of the jet- and spray-ejection velocities plotted in figure 5.6, and therefore no distinction is made here regarding the driving mechanism. The laser energy heating the vapor is estimated as the incident laser energy minus the energy required for melting of the film and heating it to the boiling temperature. This provides the energy contained by the vapor as

$$
E_{v a p}=C_{1} A_{s r f}\left((1-R) F-d L_{m}-d C_{l}\left(T_{v}-T_{0}\right)\right),
$$

where $T_{0}$ is the initial (room) temperature of the donor layer, $T_{v}$ is the boiling temperature of the donor and $C_{1}$ is a fitting factor. Equating this vapor energy $E_{\text {vap }}$ to the kinetic energy of equation (5.4) and solving the resulting equation for $V_{e j}$ yields the ejection velocity as

$$
V_{e j}=\sqrt{\frac{2 C_{1}\left((1-R) F-d L_{m}-d C_{l}\left(T_{v}-T_{0}\right)\right)}{\rho d}} .
$$

Figure 5.6 shows these calculated velocities (purple dashed lines) for copper, using $C_{1}=0.05$ and gold, using $C_{1}=0.12$. Although fitting factors are required for 
quantitative agreement, the transition from the cap- to the jet-ejection regime, which is hardly influenced by this fitting factor, is captured. The non-unity value of $C_{1}$ may be due to minor differences in the enthalpy of the vaporization $L_{m}$. The enthalpy partly consists of the energy required for the atom-by-atom escape through the liquid surface, and partly of the work done by the expanding vapor [101] which is considered as the driving mechanism. At room conditions, the work done by the expanding vapor is only $8 \%$ of the total vaporization enthalpy for both copper and gold, i.e. of similar order as the fitting factors used. Therefore, using only the work done by the expanding vapor, as for example provided by Garai et al. [101], may allow for a reasonable velocity estimate.

In the spray ejection regime, the speed of sound in air is generally exceeded by the ejected material, and ejection-induced shock waves should be present. These shock waves were observed only occasionally in the images, but were faint. Since the shock waves do not seem to influence the ejection regime nor the velocity of the ejections, and detailed visualization requires a different experimental setup, the reader is referred to literature $[26,102]$ for a detailed discussion of shock waves.

\subsection{Discussion}

Liquid phase cap ejection is scarcely described ejection regime in LIFT [103] and is therefore discussed in detail in the following. Cap ejection strongly resembles a nanobump torn off around its base [78, 104, 105]. A nanobump is a smooth bump in the metal film, which is observed below the ejection threshold fluence, which is however distinctly different from the commonly reported LIFT ejection by formation and break-up of a liquid filament [42, 48]. The dynamics of liquid phase nanobump formation were recently theoretically investigated using molecular dynamics simulations, revealing significant thinning of the donor film at the base of the bump [40]. However, cap ejection was not reported, since the simulations were limited to short timescales or sub-ejection fluence levels. However, rupture of this thin part of the film is easily conceivable and would directly correspond to the observed cap ejections. Since liquid film rupture is likely to result in the formation of multiple droplets, this mechanism may also explain the formation of satellite droplets generally observed in LIFT cap ejections [103]. The latter is further assessed in chapter 6 . Despite the multi-droplet formation, the cap ejection regime has a high potential for $3 \mathrm{D}$ additive manufacturing, since the ejected cap contracts into a single main droplet with a well-defined size and speed.

The cap ejection threshold fluence is accurately described by the capillaryinertial energy balance resulting in equation (5.7). To access the validity of this energy balance, equation (5.7) is compared to rare literature data on the liquid-film LIFT ejection. This literature includes velocity data below and above the ejection threshold. First, for numerical work on LIFT of Newtonian liquid films deposited on a dynamic release layer [24], a threshold ejection Weber number $\mathrm{We}_{e j}=1.1$ is obtained, which is in agreement with the $\mathrm{We}_{e j}=1$ criterion proposed here. Further, Young et al. [21] found no detachment of the film even for experiments performed at 
$\mathrm{We} \approx 80$. However, in that case rheological modifiers were added to the viscous film, potentially delaying film break-up as compared to Newtonian fluids. This suggest that $\mathrm{We}=1$ sets the lower bound of the minimum ejection velocity: for $\mathrm{We}<1$ retraction is expected, whereas for We $>1$ ejection occurs only if the film breaks up on a sufficiently short timescale.

Elastic stress release and partial vaporization of the film are well-established driving mechanisms for metal LIFT [35, 36, 38-40, 54]. However, the actual driving mechanisms are more complex. For example, numerical simulations by several authors revealed that cap-ejection regime is the result of a heat-induced elastic pressure wave traveling perpendicular to the film $[38-40,54]$. This wave is reflected at surface of the donor-air interface and induces pull-off of the donor from the carrier. Note that stress release towards the non-heated region of the film is limited by the speed of sound. That is, during the duration of the laser pulse, the radius of the heated area $(10 \mu \mathrm{m})$ significantly exceeds the distance the compression wave that propagates through the film $(<1 \mu \mathrm{m})$. Therefore, film buckling is not due to lateral compression, but due to the elastic [38] pressure wave reflection. These reflections have been incorporated in more comprehensive semi-analytic models, e.g. in the model of Fardel et al. [106]. Also superheating and vapor formation, expansion, and condensation could occur [60]. In this view, it is remarkable that the proposed simple energy balance in section 5.3.3 seems to capture these phenomena, although a fitting factor is required for quantitative agreement to the measurements.

Still, even for a single driving mechanism, actual observations may depend on the laser pulse duration and the film thickness. For example, for pulse durations of nanoseconds, film deformation and thermal diffusion are significant already during the pulse. Therefore, the energy deposition will be less confined and shock waves within the film could be diminished, resulting in lower ejection velocities [48]. In contrast, for femtosecond laser pulses, the energy absorption by the electrons is much faster than for picosecond pulses. However, in both cases, the energy transfer into the lattice is limited by the timescale at which the hot electrons heat the lattice given by the electron-phonon coupling timescale, which usually is in the order of 10 picoseconds. Therefore, in this short-pulse regime, lattice heating is hardly affected [107] and the proposed models (section 5.3.3) are expected to be valid. Similarly, the thickness of the film may strongly affect the observed ejection regime. In particular, if the optical penetration length is smaller than the film thickness and ejection occurs prior to thermal diffusion over the film thickness, solid-state ejection is observed [27]. In these cases, the ejection threshold depends on the yield strength of the film or its adhesion to the carrier substrate $[35,36]$. In that case, equation (5.7) no longer applies. Further discussion on the influence of the pulse duration on the LIFT process is provided in chapter 7 .

\subsection{Summary}

This chapter studied the physical conditions in copper and gold donor layers exposed to 6.7 ps laser pulses. To that end, high-resolution images of donor ejection 
during picosecond LIFT were presented. Varying the laser fluence $F$ revealed different ejection regimes, which were illustrated for a $200 \mathrm{~nm}$ copper film. For fluence levels of $320 \mathrm{~mJ} / \mathrm{cm}^{2}<F<600 \mathrm{~mJ} / \mathrm{cm}^{2}$, the ejection of a hemispherical piece of the film was observed. This is a scarcely addressed ejection regime that is referred as "cap"-ejection. For fluence levels of $600 \mathrm{~mJ} / \mathrm{cm}^{2}<F<740 \mathrm{~mJ} / \mathrm{cm}^{2}$ "jet-ejection" occurs, since here a narrow jet leading the cap apex was observed. For fluence levels of $F>740 \mathrm{~mJ} / \mathrm{cm}^{2}$ a cloud of particles was observed, called "spray ejection". Using the two-temperature model, these regimes were related to phase changes within the donor layer. In the cap ejection regime, the relaxation of elastic stresses within the melted donor film was proposed as the driving mechanism. In the jet- and spray-ejection regimes, for which the driving mechanism was found to be identical, the expansion of a vapor bubble drives the ejection. These mechanisms were well described by energy balances of the absorbed laser energy and the kinetic energy of the ejections which provided velocity predictions. Good agreement of these predictions with velocity measurements for copper and gold films was obtained, using a material-dependent fitting constant. A minimum ejection velocity of about $20 \mathrm{~m} / \mathrm{s}$ was found to correspond to a Weber number $\mathrm{We}_{e j} \approx 1$. This suggests that for lower ejection velocities at which $\mathrm{We}_{e j}<1$, surface tension retracts the liquid film. For a known velocity fitting factor, $\mathrm{We}_{e j}=1$ allows to quantitatively determine the ejection threshold fluence and vice versa. The current characterization of different LIFT ejection regimes allows for more controlled deposition of micron-sized pure metal droplets relevant for additive manufacturing. In particular, the cap ejection regime extend the range of achievable droplet sizesand velocities and will be discussed in more detail in chapter 8 . 


\section{Chapter 6}

\section{Ejection dynamics in picosecond LIFT}

In this chapter* the ejection dynamics corresponding to cap, jet and spray-like ejections are discussed. Time-resolved image sequences, supplemented by high-resolution SEM images of the donor layer, are discussed with respect to the governing physics that lead an uncontrolled ejection process during LIFT.

\subsection{Introduction}

As yet, the LIFT process has been successfully applied to transfer various materials, achieving well defined and clean depositions [63] on a receiving substrate. However, the quality of the depositions is strongly affected by the donor material and clean depositions are not routinely achieved for every material. In the case of copper, a fully clean deposition is proven to be challenging, and the governing physics related to the ejection process are still under debate, e.g. by Yang et al. [49]. Therefore, this chapter addresses the ejection dynamics during picosecond LIFT of copper. Here, in particular the generation of occasional defects, i.e. contamination of the receiver due to the impact of multiple small droplets instead of a single droplet, is investigated. So far, the majority of experimental results reported in literature are based on post process analysis, i.e. the inspection of the deposited droplets on the receiving substrate or the donor layer [12-17]. In literature, two main theories $[36,41]$ are proposed to explain the ejection of the donor on the basis of the relaxation of thermally induced stresses, or the creation of a vapor/plasma bubble at the carrier donor interface, see also chapter 5 . In this context, also

${ }^{*}$ Parts of this chapter have been submitted for publication as R. Pohl, C.W. Visser, G.R.B.E. Römer, C. Sun, D. Lohse. Characterization of the cap ejection regime in laser-induced forward transfer of copper. 
the violent ejection mechanism of explosive boiling is often discussed [18, 108]. Obviously, explosive boiling seems to be a reasonable explanation for the observed contamination during high fluence ejections. However, it is unknown at which conditions these phenomena occur and whether explosive boiling can also explain the contamination during low fluence LIFT. To answer this question, high-resolution imaging is proven to be a suitable method to provide insights in the ejection dynamics during LIFT. However, as yet time-resolved observations of LIFT processes of $\mathrm{Au}$ [31], $\mathrm{Ni}$ [32] and $\mathrm{Cr}$ [34] do not achieve sufficient spatial resolution to follow the dynamics of the ejection process in detail and to identify the origin of the contamination.

Therefore, this chapter presents an experimental study on the ejection dynamics of copper-based picosecond LIFT. First, the experimental methods are briefly discussed in section 6.2. Next, in section 6.3 high-resolution time-resolved image series are presented in order to investigate the dynamics of cap, jet and spray like ejections during LIFT, which were prior discussed in chapter 5 . Those image series are complemented by high-resolution SEM images that are provided to relate the observed ejection dynamics to the physical conditions in the donor layer. The latter are calculated on the basis of the two-temperature model described in section 3.3. Next, for each ejection regime these findings are summarized in a hypothesis that provides a qualitative description of the corresponding ejection process. Finally, conclusions are presented in section 6.4.

\subsection{Methods}

The experiments to obtain time-resolved image sequences were performed with the same experimental setup, as used for the classification of the ejection regimes, discussed in section 5.1. However, in order to investigate the ejection dynamics during LIFT, the combination of the dual-shot camera with the dual-cavity strobe illumination source was utilized to follow the ejection process in time in more detail. That is, each ejection event has been imaged twice, referred to in the following as "image A" and "image B". The temporal delay between these A- and B-images was controlled via the pulse delay-generator and was set to $350 \mathrm{~ns}$.

\subsection{Results}

\subsubsection{Cap ejection}

\section{Donor layer conditions}

Figure 6.1 shows SEM images of an ejection crater in a $200 \mathrm{~nm}$ thick copper layer after a cap ejection at a fluence level of $444 \mathrm{~mJ} / \mathrm{cm}^{2}$. Figures 6.1 (a) and 6.1 (b) provide an overview of the same crater formed after cap ejection, but at two different viewing angles. Figures 6.1 (c) and 6.1 (d) provide details of this ejection crater as they show a close-up of the inner area of the crater 6.1 (c) and the rim 6.1 (d), that 


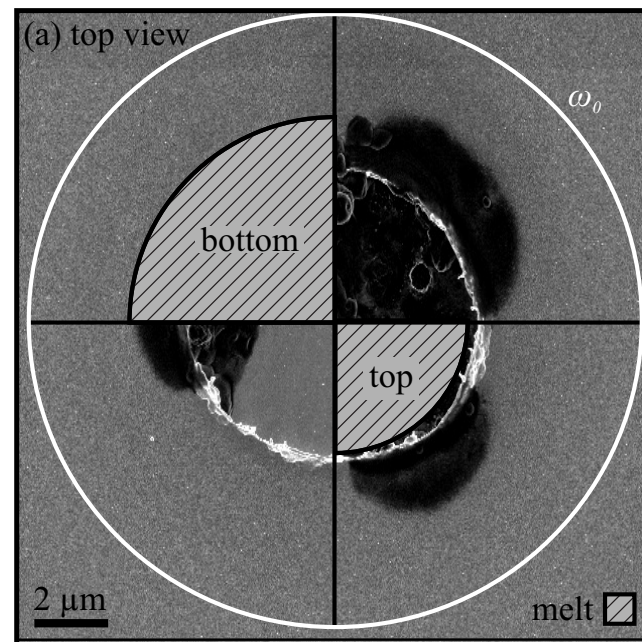

(b) tilted $45^{\circ}$

(c) recrystallized copper

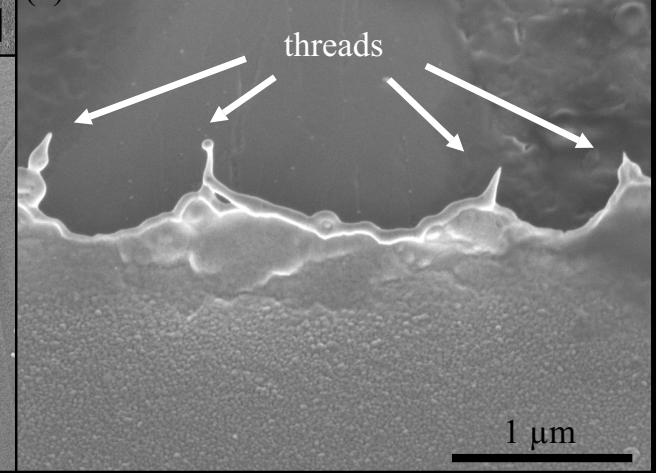

Figure 6.1: SEM images of the ejection crater in a $200 \mathrm{~nm}$ thick copper donor layer obtained a at laser fluence of $444 \mathrm{~mJ} / \mathrm{cm}^{2}$. (a) Overview of the ejection crater (top view) supplemented with the computed temperature distribution, indicating the physical conditions of the donor layer. The white circle indicates the spot size of the laser. (b) Tilted view on the ejection crater. (c) Detailed view on the interior of the ejection crater, indicating the crystal structure of the resolidified donor layer, remaining at the carrier. (d) Close-up of the rim formation at the edge of the crater and indications of prior liquid threads along the rim.

was formed during the detachment of the cap.

For comparison, the donor temperatures at the carrier-donor interface (bottom) and at the donor-air interface (top), computed using the model introduced in section 3.3, are plotted on-top of figure 6.1 (a). For a qualitative analysis, the calculated temperatures have been consolidated into two regimes, separated by the temperatures of melting and vaporization, at equilibrium conditions. This allows for a simple estimate of the physical conditions in the donor layer. The computed results support the qualitative impression of the SEM images. Namely the inner area of the crater fully corresponds to the full melting regime of the donor layer. The hatched overlay suggests that the break-up area, as suggested by the shape of the rim, corresponds to 
the full melting threshold of the donor layer. However, the outer solid area is heated by the laser pulse as the Gaussian profile decays along the radius, but is not melted nor ejected during the ejection process.

It is remarkable that the area inside the crater seems to be covered fully with a thin remaining layer of copper. The detailed view of figure 6.1 (c) clearly shows a thin layer of resolidified copper inside of the crater. The thickness of this layer was measured to be about $100 \mathrm{~nm}$, using the confocal microscope. This phenomena is inline with the assumption of stress related ejection. That is, stress related ejection of a transversal propagating stress wave inside of the donor layer. As shown in related work by Shugaev et al. [54], such a stress wave will peak at almost half the layer thickness. As a result, the layer is chipped at that location and parts of the donor layer are not ejected bu will partly remain on the carrier. This provides evidence, that the ejection process is sufficiently described by a $1 \mathrm{D}$ approach, where the driving force is directly proportional to the geometrical shape of the Gaussian beam profile of the focused laser pulse. The crystal structure observed in figure 6.1 (c) is attributed to the cooling conditions of the donor. It is assumed that the remaining copper layer cools down at relatively slow cooling-rate, which is dominated by the heat conduction to the carrier and convective heat conduction towards the air interface. As both boundaries exhibit a rather low heat conduction, the remaining copper solidifies rather slowly and consequently well defined crystals are formed.

Next, the rim formation as shown in figure 6.1 (d) shows features referred to here as "threads". These threads are frequently observed along the rupture rim. The rupture line and these thread like formations give a strong evidence of prior molten copper as these features are known from liquid sheet break-up experiments [109]. The observed threads will be investigated in more detail in the following.

\section{Ejection dynamics}

Figure 6.2 shows several cap ejection phenomena obtained at fluence levels ranging from $360 \mathrm{~mJ} /$ to $520 \mathrm{~mJ} / \mathrm{cm}^{2}$. The ejections were captured at two time instances of $50 \mathrm{~ns}$ and $400 \mathrm{~ns}$ with respect to the time instance of the first visible deformation of the donor layer. Figure 6.2 (a) shows a small cap that is partly detached from the donor layer. With increasing laser fluence levels, the heat affected zone in the donor layer and consequently also the volume of the ejected cap increases with the Gaussian shape of the focused laser pulse, as shown in figures 6.2 (a) to 6.2 (g). Figures 6.2 (d) and 6.2 (e) represent typical examples of half-spherical shaped cap ejections. For higher fluence levels, as indicated in figure $6.2(\mathrm{~g})$, the leading edge of the cap is slightly disrupted and the indication of a small jet is visible.

The width of these caps was found to vary from $3 \mu \mathrm{m}$ to $10 \mu \mathrm{m}$ and matches with the observed crater formation at the donor-air interface of the donor layer, shown in figure 6.1. The ejected volume of the ejected cap is estimated from the donor layer thickness and the observed crater diameter. This volume is compared to the observed ejections, which can be estimated by the geometry of the ejected cap. A volume comparison reveals significant mismatch of one order of magnitude. Hence, it is likely to assume that at this instance the ejections are not solid but 


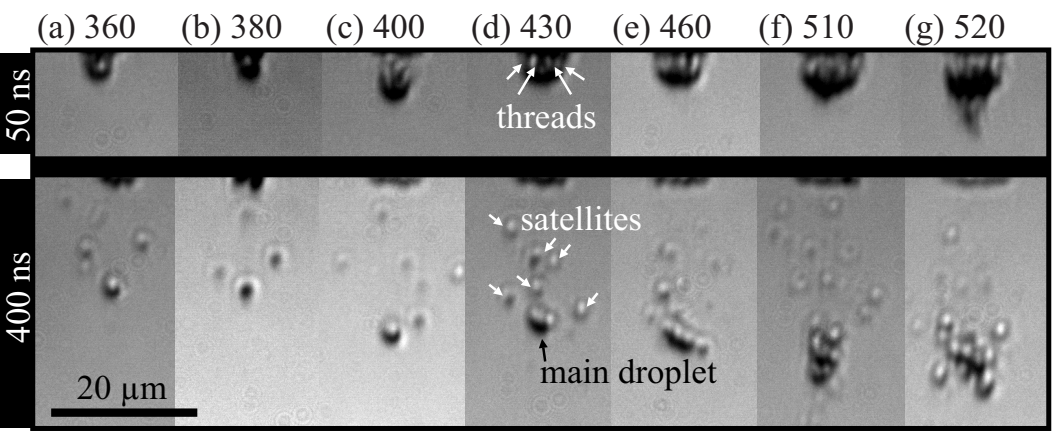

Figure 6.2: Side view of cap ejection events at time instances of $50 \mathrm{~ns}$ (top row) and $400 \mathrm{~ns}$ (bottom row) after the start of the laser pulse, obtained at various fluence levels corresponding to the cap ejection regime. In image (d) liquid threads between the donor layer and the cap during the ejection process are indicated by arrows, as observed at the time instance of $50 \mathrm{~ns}$. The time instance of $400 \mathrm{~ns}$ shows multiple droplets, which are identified either as satellites or as a main droplet. Here, the main droplet refers to the corresponding droplet, which is considered to represent the contracted droplet of the ejected cap.

hollow and the cap resembles the ejection of an "umbrella" like ejection of a thin liquid sheet of molten copper.

It is remarkable that the caps at this time instance, seem to be partly attached to the donor layer, as thin "threads" between the cap and the donor layer are visible. These threads are indicated in figure 6.2 (d) by arrows. The formation of these threads are frequently observed during the experiments and remnants of those are also found in the SEM images of the ejection crater, see figure 6.1 (d).

At the second time instance (at $400 \mathrm{~ns}$ ) the initially well defined cap ejections are contracted and many relatively small droplets are observed. Typically, those observations consists of a main droplet, which is identified as the contracted cap, and additional smaller droplets, referred to as "satellites". The latter are part of speculation as their origin is not clarified yet. However, from the presented observations it is clear that the break-up of the cap happens on a time-scale of 350 ns. These kind of thread formations are often discussed in the context of capillary thinning and break-up, referred to as "necking" [110]. In this context several theories regarding the thinning dynamics have been developed, in order to explain the temporal evolution of the thread radius until break-up. Assuming a Newtonian fluid and a constant cap ejection velocity, the critical time to break-up is estimated as

$$
t_{c}=14.1 \eta \frac{r_{0}}{\sigma}
$$

with the density $\rho=8.96 \mathrm{~kg} \mathrm{~m}^{-3}$, the surface tension $\sigma=1.25 \mathrm{~N} \mathrm{~m}^{-1}$ [111], viscosity $\eta=5 \cdot 10^{-3} \mathrm{~N} \mathrm{~s} \mathrm{~m}^{-2}[112]$ and an estimated initial radius of $r_{0}=1 \mu \mathrm{m}$, to be 15 ns. This timescale is therefore matching the presented observations. If the satellites originate from the threads, the observed number of satellites should correspond to the number of threads visible along the rupture. This is discussed in detail in the 


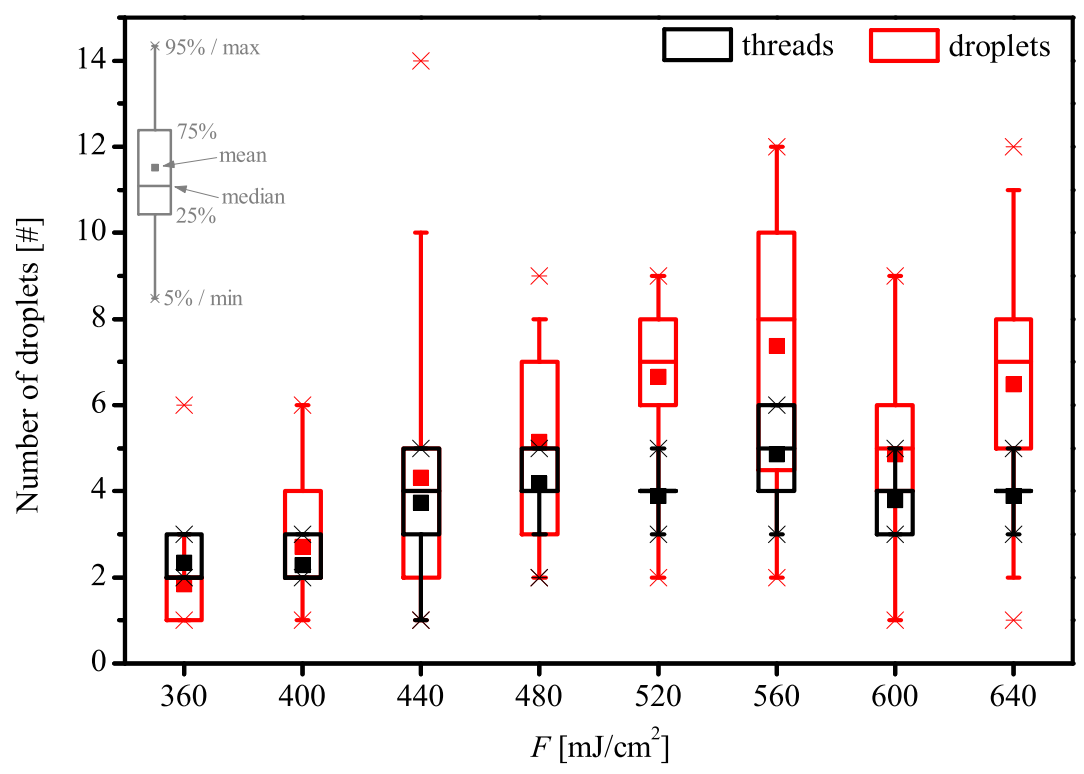

Figure 6.3: Box-plot that summarizes the statistical analysis of cap ejections for laser fluence levels from $340 \mathrm{~mJ} / \mathrm{cm}^{2}$ to $650 \mathrm{~mJ} / \mathrm{cm}^{2}$, as shown in figure 6.2 . The fluence dependent evolution of the observed number of threads (black) during the ejection process at a time instance of $50 \mathrm{~ns}$ (A-image), and the number of generated droplets (red) at a later time instance of $400 \mathrm{~ns}$ (B-image) show a significant correlation for the low fluence interval ranging from $360 \mathrm{~mJ} / \mathrm{cm}^{2}$ to $480 \mathrm{~mJ} / \mathrm{cm}^{2}$.

\section{following.}

\section{Ejection statistics}

Further investigations on the formation and break-up of the liquid threads during the cap ejection are based on 220 image pairs (A- and B-images) that have carefully been analyzed. That is, from each image the following data was extracted: crater location and diameter, number of threads at the rupture, cap location and the number and location of satellites. Figure 6.3 shows a box-plot that summarizes the statistical data obtained. The color refers to the A- and B- images and correspond to the observed number of threads and satellites at the given time instance for different laser fluence levels, binned with a fluence interval of $40 \mathrm{~mJ} / \mathrm{cm}^{2}$. Despite the relatively large variation in both data sets, a trend of an increased number of threads and satellites towards higher fluence levels can be identified. Moreover, in the low fluence interval from $360 \mathrm{~mJ} / \mathrm{cm}^{2}$ to $480 \mathrm{~mJ} / \mathrm{cm}^{2}$, both data sets show a significant correlation. That is, the number of threads and the observed number of satellites during the ejection are related. For higher fluence values the data loses its validity and no significant trend is observed. This might be explained by the transition of the cap ejection 


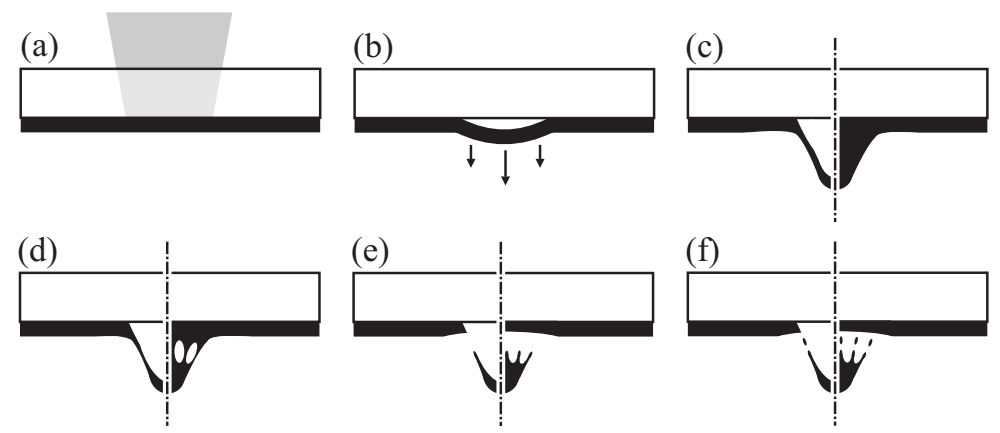

Figure 6.4: Illustration of several stages in the stress-driven cap ejection mechanism. (a) Absorption of the laser pulse within the optical penetration depth of the donor layer, resulting in a temperature gradient (not shown) collinear to the optical axis. (b) Donor layer deforms in a cap like shape, resembling the geometrical shape of the Gaussian laser pulse. (d) The bubble emerges and the elongation of the film leads to a local dependency of the film thickness on the displacement profile. (e) The cap detaches and liquid threads are formed along the rupture. Due to the stochastic behavior of the break-up of the threads either a single droplet (e) or multiple droplets (f) are generated.

regime towards the jet ejection regime, which was already discussed in section 5.3. In addition, the number of single droplet ejections has been investigated and found to be the minority of ejection events with $14 \%$, even at the ejection fluence threshold. In contrast, the majority of $86 \%$ of the images show multiple droplets.

From these results it is concluded, that origin of the observed satellites is an in-flight phenomena, which strongly suggest the break-up of liquid threads as the governing mechanism. Moreover, the presented results introduce a novel explanation regarding the generation of satellite droplets during printing of metals using LIFT.

\section{Driving mechanism of cap ejection}

Based on the investigations summarized in the prior section and the results presented in chapter 5, a qualitative description of the ejection process for low fluence LIFT is proposed and illustrated in figure 6.4. The ejection process is related to the full melting of the donor layer and it is assumed that the copper is ejected in liquid phase, whereas the inertial flow inside the liquid film is neglected. First, the ejection is initiated by the absorption of the laser pulse and the subsequent heating and stress build-up in the donor layer, see figure 6.4 (a). The thickness of the donor layer exceeds the penetration depth of the incident laser pulse and a temperature gradient collinear to the optical axis is established. A transversal stress wave is induced that propagates collinear to the optical axis. Next, in figure 6.4 (b) the stress wave relaxes as the donor layer is ejected in a cap like shape, resembling the geometrical shape of the Gaussian laser pulse. The expansion of the ejected film is hampered, due to the zero pressure bubble, that is generated inside of the emerging copper bubble. 


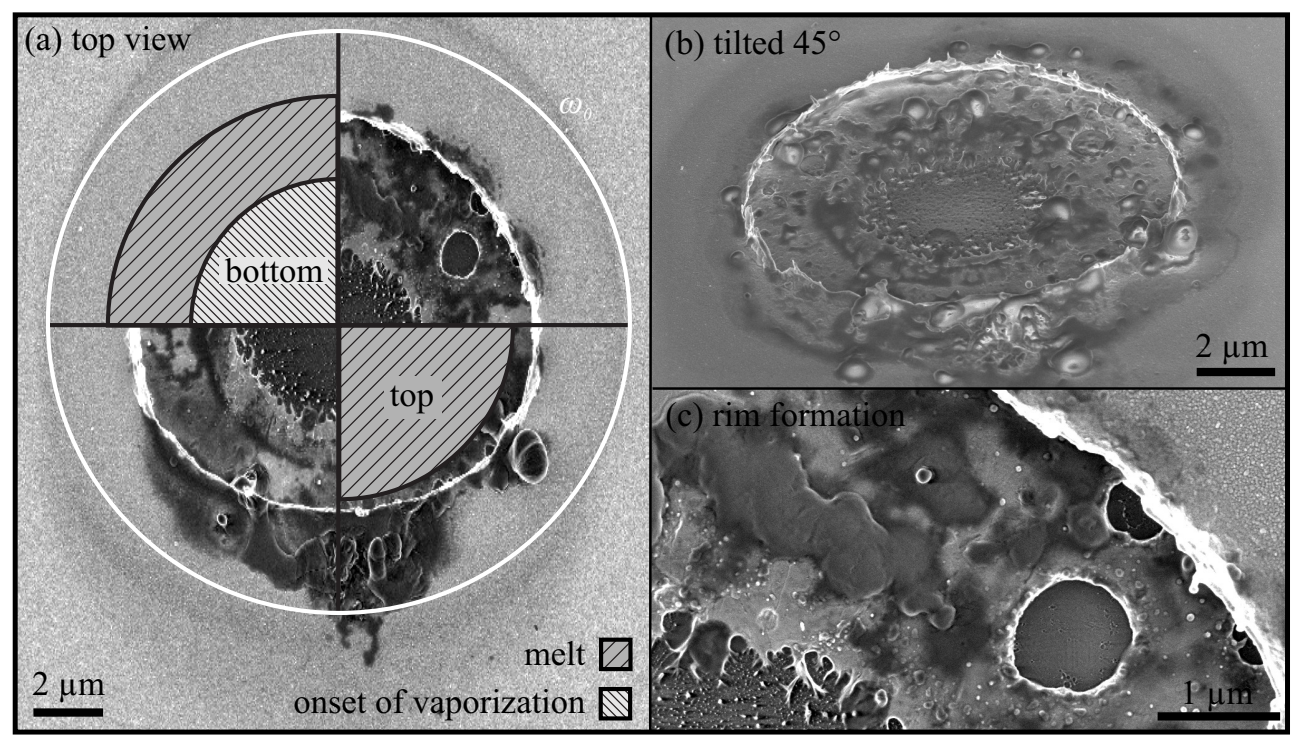

Figure 6.5: SEM images of the ejection crater in a $200 \mathrm{~nm}$ copper donor layer, obtained at a laser fluence of $770 \mathrm{~mJ} / \mathrm{cm}^{2}$. (a) Overview of the ejection crater at normal incidence. The physical conditions of the donor layer at the carrier-donor interface (bottom) and the donor-air interface (top) are plotted on top of the SEM image. The white circle indicates the spot size of the laser. Overview image of the ejection crater at $45^{\circ}$ (b) and details of the rim formation (c).

However, the ejection process is still dominated by the inertia of the initial stress wave and liquid motion as described by Unger et al. [84] is neglected. As the liquid copper bubble emerges, the elongation of the film depends on the slope of the profile. This leads to a local dependency of the film thickness on the slope of the displacement profile, figure 6.4 (c). The cap detaches from the donor layer at the location, where the film thickness reaches a critical layer thickness. Similar to the ejection in liquid sheet experiments, the rupture of the cap leads to the formation of liquid strings between the cap and the donor, referred to here as threads, see figure 6.4 (d). Now, two scenarios are observed: 6.4 (e) the rupture and subsequent ejection of the cap leads to the explained thread formation, but during break-up the threads stick either to the main droplet or the rim of the crater in the donor layer and no additional droplets are generated. Or, as shown in figure 6.4 (f) the break-up of the threads leads to the formation of additional droplets, which are ejected along with the main droplet, as described in figure 6.3. 


\subsubsection{Jet ejection}

\section{Donor layer conditions}

Figure 6.5 shows SEM images of an ejection crater in a $200 \mathrm{~nm}$ thick copper layer after jet ejection at a fluence level of $770 \mathrm{~mJ} / \mathrm{cm}^{2}$. Figures 6.5 (a) and 6.5 (b) provide an overview of the ejection crater after jet ejection, at two different viewing angles. Figure 6.5 (c) shows a detailed view of this ejection crater and provides a close-up of the inner area of the crater and the rim, that was formed during the detachment of the jet from the donor layer.

For a qualitative analysis, the consolidated temperatures of the donor layer at the carrier-donor interface (bottom) and the donor-air interface (top) are calculated and plotted on-top of figure 6.5 (a). Here, the computed results support the qualitative impression of the SEM images. That is, the central area of the ejection rater corresponds to the onset of vaporization of the donor layer, suggesting the formation of a vapor bubble. The vapor bubble is fully enclosed by a thin layer of molten donor, as the onset of vaporization does not exceed the thickness of the donor layer. The area between the vapor bubble and the rim formation corresponds to the full melting of the donor layer. Similar to the observations in the cap regime in section 6.3.1, a thin layer of donor material remains on the carrier interface. Also, the computed overlay suggests that the break-up area as indicated by the rim formation, corresponds to the full melting threshold of the donor layer. In addition, the outer solid area seems to be affected by the laser pulse as the Gaussian profile "decays" along the radius, but is not melted nor ejected during the ejection process.

As discussed in section 5.3.3, the jet ejection regime is interpreted as a vapor driven ejection regime. However, it may be hypothesized that the balance of the driving forces is reflected in an area balance between the vapor dominated area indicated by the full removal of the donor layer and the stress-driven force, which is correlated to the remaining donor at the carrier interface. In figure 6.5, the corresponding areas are of the same order of magnitude. Hence, at this point the jet regime may be reinterpreted as a transition regime from the stress driven cap ejection towards the vapor dominated spray ejection regime.

\section{Ejection dynamics}

As the LIFT process is highly dynamic in the jet-fluence regime, time-resolved measurements within this fluence regime were performed. Figure 6.6 shows timeresolved images of the LIFT process at a laser fluence of $806 \mathrm{~mJ} / \mathrm{cm}^{2}$. The initial speed of the tip of the jet was estimated to be $250 \mathrm{~m} / \mathrm{s}$. The time delay between the images was set to $50 \mathrm{~ns}$ for figures 6.6 (a) to 6.6 (d), and to $100 \mathrm{~ns}$ for figures 6.6 (e) and 6.6 (f), respectively. Initially, the copper layer deforms into a dome with a roughly spherical shape, figure 6.6 (a). Subsequently, this dome is elongated and detaches from the donor layer, figure 6.6 (b). Next, the shape of the ejection changes drastically. In figure 6.6 (c) two characteristic aspects of this ejection can be observed. First, a "shoulder" that has hardly deformed compared to figure 6.6 (b) and secondly, a slender jet that has strongly deformed. The shoulder and 


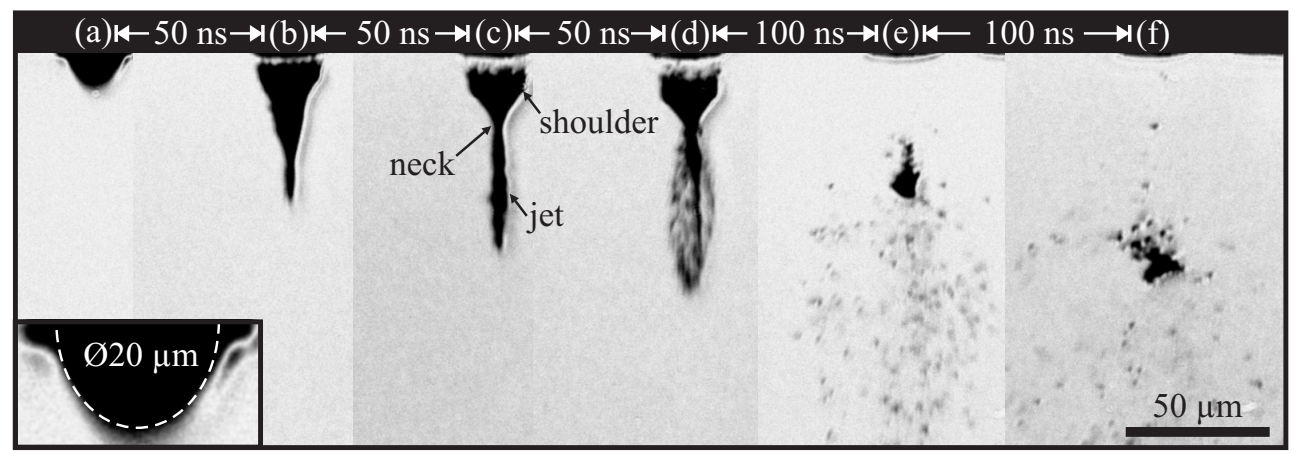

Figure 6.6: Time-resolved image sequence of the ejection process obtained at a laser fluence of $806 \mathrm{~mJ} / \mathrm{cm}^{2}$. A liquid copper jet is formed on the donor-air interface of the donor layer. Image (a) shows the deformation of the donor layer and the forming of a roughly spherical dome. Image (b) shows the elongation and detachment of this dome, forming a jet. Subsequently, the ejected jet contracts, forming a neck (c), that fragments (d). Images (e) and (f) show the disintegrated jet, resulting in a main droplet accompanied by a cloud of particles. The insert shows a close-up view of (a), indicating the estimated diameter of the observed dome.

jet are separated by a "neck", where the contraction of the jet is strongest. Over time, the shoulder "gently" contracts to form a main droplet. In contrast, the jet implodes into many relatively small droplets or fragments. This mechanism explains the massive contamination of a receiver previously reported for high-fluence LIFT experiments [108] and therefore complements the proposed explosive boiling mechanism as a source of contamination. These observations of the characteristics of the jetting regime show similar aspects as discussed for jet ejections in liquid-film LIFT [22] and experiments with liquids [113]. However, the reported jets in this section are assumed to be hollow at the initial state and the formation process is assumed to be fundamentally different. Accordingly, the fragmentation of the jet may be explained by liquid sheet break-up as will be described in the following.

\section{Driving mechanism of jet ejection}

As was shown in chapter 5 , jet-like ejections are found to be an intermediate regime between the cap- and the spray-like regime. The formation of a jet was observed for laser fluence levels at which the onset of vaporization at the carrier-donor interface was computed. These results were supported by the observations of the donor layer and the ejection dynamics, as given by the time-resolved image sequence. Moreover it was proposed, that the jet formation will occur when the onset of a vapor bubble, covered by a shell of molten copper is initiated. Figure 6.7 shows a schematic illustration of several stages of the jet ejection during the LIFT process. Based on the current results, it is assumed that the copper is in a liquid phase and partially vaporized. A $1 \mathrm{D}$ approach is assumed and the inertial flow inside the liquid film is neglected. First, to explain the formation of the neck, the ejected liquid copper film is considered. 


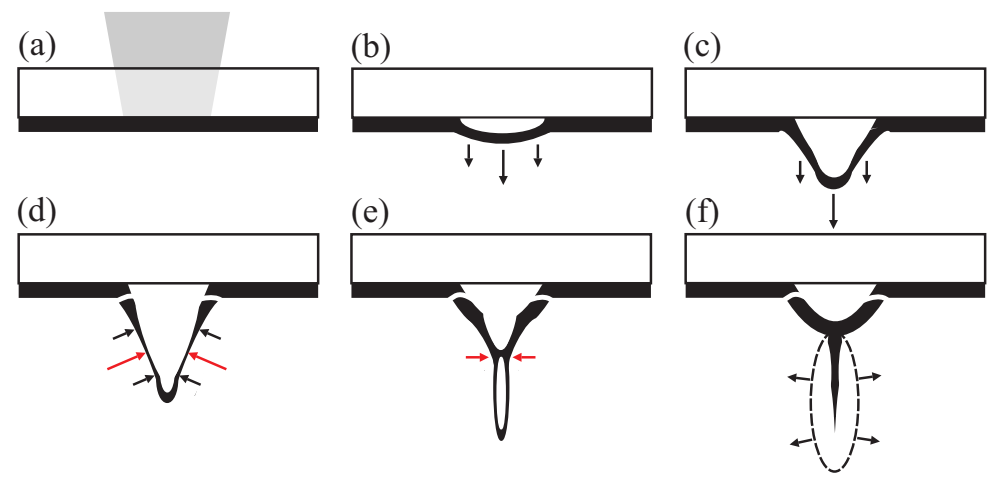

Figure 6.7: Schematic illustration of several stages in the jet ejection mechanism during picosecond LIFT. (a) Laser pulse absorption in the donor layer leading to locally melting and onset of vaporization of the donor layer. (b) Expansion of the copper bubble due to stress relaxation. (c) Further expansion elongates the liquid film and leads to locally thinning of the liquid film. (d) Contraction of the cone with locally different velocities, that are correlated to the film thickness. (e) Entrapment of a bubble below the neck, that is assumed to be located at the former thinnest part of the liquid film. (f) Fragmentation of the jet caused by the implosion and break-up of the enclosed bubble.

It is assumed that the initial velocity of the cap is perpendicular to the surface and proportional to the geometrical shape of the Gaussian profile of the focused laser pulse, see figure 6.7 (b). The shape of the cap is approximated with a Gaussian displacement profile, figure 6.7 (c). However, the center of the cap is accelerated faster, due to the local vaporization of the donor layer. As the copper bubble emerges, the elongation of the film depends on the slope of the velocity profile. This leads to a local dependency of the film thickness on the slope of the displacement profile, figure 6.7 (d). Thus, if the gradients of the driving forces i.e. surface tension and/or pressure over the liquid film are negligible, thinner parts will accelerate faster than thicker parts. As the jet contracts, a neck is likely to form in the thinnest area, see figure 6.7 (e). The closure of the neck will result in the entrapment of a bubble. Hampered by the enclosed vapor, the contraction of the remaining bubble ultimately leads to the break-up of the remaining bubble, leading to the observed fragmentation.

\subsubsection{Spray ejection}

\section{Donor layer conditions}

Figure 6.8 shows images of the ejection crater obtained at a laser fluence of 1300 $\mathrm{mJ} / \mathrm{cm}^{2}$, corresponding to a fluence level within the spray ejection regime. Figures 6.8 (a) and 6.8 (b) show SEM images of the crater at two different viewing angles. Figure 6.8 (c) shows a SEM image of a cross-section of the ejection crater prepared with a focused ion beam. To prevent tempering effects during the preparation of the sample with the FIB, the area of interest was covered with multiple layers of platinum, 


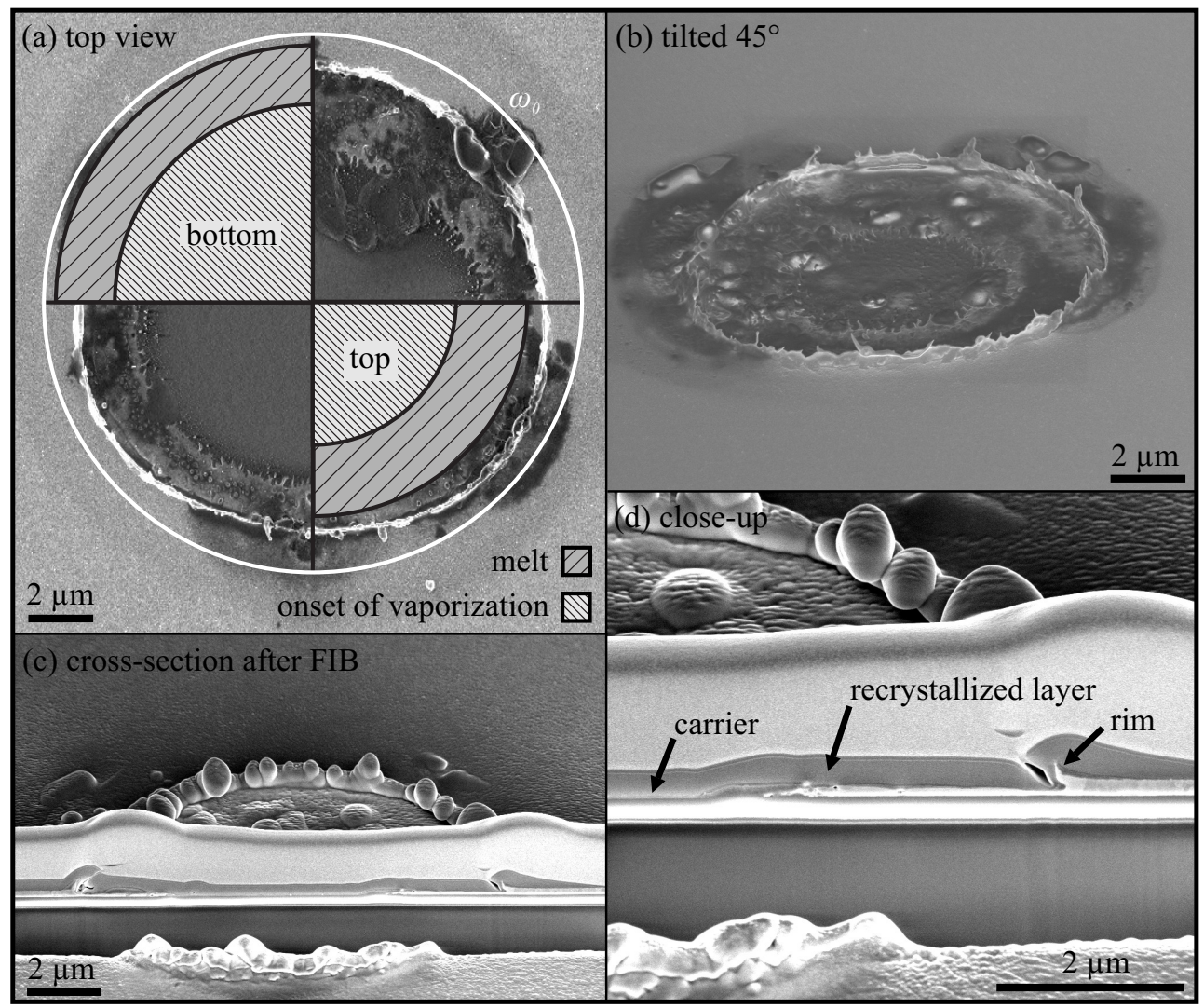

Figure 6.8: SEM images of the ejection crater in a $200 \mathrm{~nm}$ copper donor layer obtained at a laser fluence of $1300 \mathrm{~mJ} / \mathrm{cm}^{2}$. (a) Shows an overview image of the ejection crater supplemented with the computed physical conditions in the donor layer. The white circle indicates the spot size of the laser. (b) Shows the ejection crater at an viewing angle of $45^{\circ}$. (c) SEM image of a cross-section of the same ejection crater as shown in (a) and (b). (d) Detailed view on the cross-section showing the recrystallized layer in the interior of the ejection crater and the rim formation, which is related to the full melting of the donor layer.

which are visible on top of the ejection crater. A close-up of this cross-section provides details about the rim formation and the interior of the ejection crater, shown in figure $6.8(\mathrm{~d})$.

The ejection crater shown in figures 6.8 (a) and 6.8 (b) appear to be similar to the craters that are observed for laser fluence levels within the jet ejection regime. That is, both regimes indicate the formation of a vapor bubble in the center of the ejection crater. However, for fluence levels in the spray regime, the area attributed to the formation of a vapor bubble is more prominent and dominates the interior of the crater. The computed temperature distribution supports the qualitative impression of the SEM image. That is, the center of the crater reached a critical state, as the 


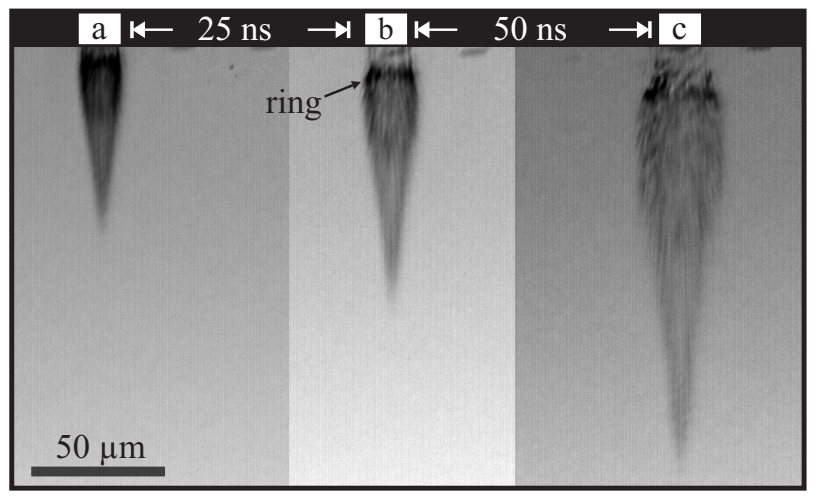

Figure 6.9: Time-resolved images sequence showing the spray ejection at a laser fluence of $1300 \mathrm{~mJ} / \mathrm{cm}^{2}$. The ejected spray expands with an ejection speed of $\approx 900 \mathrm{~m} / \mathrm{s}$ (a) and a ring-like feature (b) and (c) is formed at the trailing edge of the spray ejection.

whole layer thickness reaches the boiling temperature, as indicated by the onset of vaporization at the donor-air interface of the donor layer. As a consequence, the ejection process at this point is considered to be dominated by the vaporization of the donor layer and as a result the influence of the stress relaxation becomes negligible.

The cross-sections in figures 6.8 (c) and 6.8 (d) provide details of the ejection crater profile. Three features are identified. The interior of the crater corresponds to the onset of vaporization and the formation of a vapor bubble. The vaporized area is characterized by the full removal of the donor layer. The outer area of the interior of the crater is related to the stress driven ejection mechanism. Here, the remaining donor layer is clearly visible. The layer thickness obtained from the SEM images match with the prior made measurements using the confocal microscope and is measured to be about $100 \mathrm{~nm}$, which corresponds to half the layer thickness. The third detail is found at the rim of the ejection crater, which shows the deformation and thinning of the donor layer. Besides the indication of resolidified threads at the top of the rim, also a minor plastic deformation from the bottom the top of the copper layer can be observed.

\section{Ejection dynamics}

Figure 6.9 shows a time-resolved image sequence obtained at a fixed laser fluence of $1300 \mathrm{~mJ} / \mathrm{cm}^{2}$. The time delay between these images was set to $25 \mathrm{~ns}$ and $50 \mathrm{~ns}$ between the 6.9 (a) and 6.9 (b), and 6.9 (b) to 6.9 (c), respectively. The image sequence shows the evolution of the ejected spray of particles at ultra-high speed with a velocity of $\approx 900 \mathrm{~m} / \mathrm{s}$. Accordingly, motion blur limits the resolution of the images and a spray of undefined particles is observed in figure 6.9 (a). The ejected spray expands over time and a characteristic "ring" that is formed at the trailing edge of the spray ejection is observed, figures 6.9 (b) and 6.9 (c). The velocity of these rings is measured to be about $60 \mathrm{~m} / \mathrm{s}$ and therefore corresponds to the ejection 
velocities measured for stress driven cap ejection. At this point it may be argued, that the observed rings comprises of the ejected liquid donor, which is driven by stress relaxation, as discussed in section 6.3.1.

Note that, despite the high ejection velocity, the images do not indicate the formation of a shock wave. The absence of a shock wave is attributed to the optical layout of the imaging setup. This setup is not sensible to detect changes in the refractive index of the ambient atmosphere. Although shock waves have occasionally been observed during the experiments, but were faint and hardly reproducible. For further discussions on shock waves during LIFT, the reader is referred to Fardel et al. [114].

The observed atomization in the form of a spray like ejection obviously results in a massive contamination the receiving substrate and is likely to be explained by the phenomena of explosive boiling [115]. This is strongly supported by the velocity and the temporal evolution of the observed spray ejection, as it shows many similarities to the ablation mechanism, as studied in detail by Zhigilei et al. [116]. Similar to the observations in figure 6.9, the modeled ablation process in that article consist of multiple phases, which were attributed to spallation at the periphery of the Gaussian beam profile and explosive boiling for center of the laser pulse. However, the topics of laser ablation and explosive boiling/phase explosion are complex and rarely studied for the laser irradiation conditions typical for LIFT. A more conclusive description of this regime is unfortunately out of the scope of this thesis.

\subsection{Summary}

In this chapter, the origin of undesired contamination during picosecond LIFT was addressed. To this end, SEM images of the ejection craters after LIFT, and timeresolved image sequences at laser fluence levels corresponding to the cap, jet and spray ejection regimes were discussed. Figure 6.10 provides an overview of the ejection dynamics as observed during LIFT of copper and gold. The donor layer conditions for different fluence values are indicated on top of figure 6.10. Depending on the laser fluence either cap, jet or spray ejections were observed. The ejection dynamics are indicated by the characteristics of each ejection regime at the time instances of $50 \mathrm{~ns}$ and $400 \mathrm{~ns}$. The quality of the deposited features is indicated at the bottom of the figure, in terms of "clean" (single droplet) or "contaminated" (multiple droplets).

For cap ejection, the generation of additional undesired droplets was found to be correlated to the separation of the ejected cap from the donor layer. That is, the subsequent formation and break-up of liquid threads along the rupture line of the ejected cap were proposed to cause the formation of additional droplets, next to the ejected cap. Moreover, the driving mechanism of the ejection process were discussed in more detail to complement the findings, presented in chapter 5 . The analysis of the ejection crater show a remaining copper layer in the interior of the ejection crater. From that, the the generation of a transversal stress wave [54] is proposed and a 1D ejection process is assumed. Accordingly, the shape of the ejected caps was proposed to resemble the geometrical shape of the Gaussian profile of the focused laser pulse. 


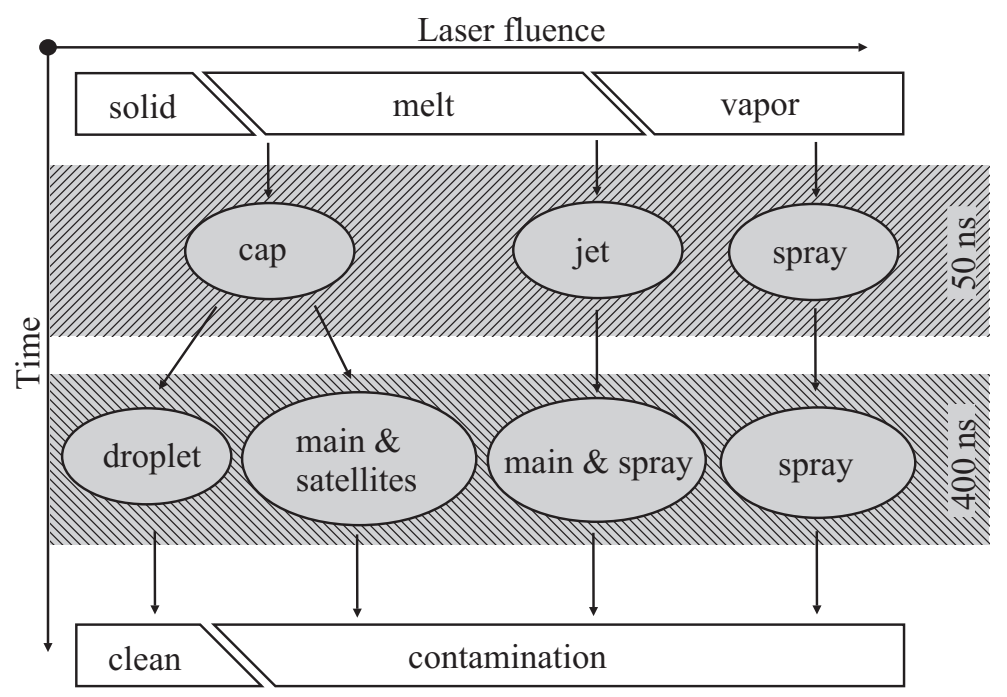

Figure 6.10: Schematic illustration of the ejection dynamics during laser-induced forward transfer. Depending on the laser fluence and the correlated physical conditions in the donor layer either cap, jet or spray-like ejections are observed. The ejection dynamics result in the generation of either a single droplet (clean) or multiple droplets (contamination) which are deposited on a receiving substrate on impact.

To this end, a qualitative description of the cap ejection process was provided.

Next, the jet ejection regime was assessed following the proposed 1D approach. From that, it was shown that the absorbed laser pulse ultimately causes a velocity gradient across spatial profile of the ejected jet. This profile was attributed to the geometrical shape of the Gaussian beam profile and the onset of vaporization in the center of the ejection crater. The proposed ejection mechanism provided a reasonable explanation for the dynamics that ultimately lead to the full fragmentation of the ejected jets. This fragmentation leads to the formation of many relatively small droplets and was shown to be key to explain the massive contamination in this fluence regime.

Finally, the ejection dynamics of the spray ejection regime were discussed. It was found that the captured ejection dynamics resemble the characteristics of explosive boiling, as discussed in prior work by Zhigilei et al. [116]. To this end, the donor ejection due to homogenous melting was considered and the spray ejection during picosecond LIFT was fully attributed to the dominance of explosive boiling. 



\section{Chapter 7}

\section{Ejection phenomena in nanosecond LIFT}

In this chapter* the driving mechanism during nanosecond LIFT and the effect of the pulse duration on the observed ejection regimes are discussed. The results complement the picosecond results (discussed in chapters 5 and 6) and are used for a general discussion on the driving mechanism during LIFT, in particular the dependency of the observed ejection regimes on the pulse duration.

\subsection{Introduction}

The experiments on nanosecond LIFT were performed in order to allow for a discussion of the influence of the laser pulse duration on the ejection process during LIFT. In this thesis, the ejection regimes and the corresponding ejection dynamics during picosecond LIFT have been discussed in detail in chapters 5 and 6 . It was found that for laser fluence values at the transfer threshold, the donor layer is ejected due to the stress buildup in the donor layer, which ultimately leads to the ejection of a cap like film - contracting into a droplet on the time-scale of 100's of nanoseconds. In contrast to those observations, imaging studies on copper of nanosecond LIFT[48] indicate a significantly different ejection mechanism, which is characterized by the subsequent formation and break-up of a liquid jet, instead of a cap ejection for laser fluence values just above the transfer fluence threshold. Those experiments were carried out using a rather thick donor layer with a layer thickness of $600 \mathrm{~nm}$ and a laser pulse duration of $15 \mathrm{~ns}$. Surprisingly, similar results were obtained with femtosecond laser-induced forward transfer of gold [42], using a

*Parts of this chapter have been published in Journal of Laser Micro/Nanoengineering, 10(2):154-157, 2015, R. Pohl, C.W. Visser, G.R.B.E. Römer, C. Sun, A.J. Huis in 't Veld, and D. Lohse. Imaging of the ejection process of nanosecond laser-induced forward transfer of gold. 
layer thickness of $60 \mathrm{~nm}$. Also here, it was found that, for laser fluence levels just above the ejection transfer threshold, the formation of a liquid jet is observed. The ejection mechanism was explained by the authors as the hydrodynamic behavior of the molten donor layer, which ultimately leads to the break-up of the ejected jet into a single and/or multiple droplets.

However, an extensive description of LIFT is missing and in particular detailed studies on the influence of the laser pulse duration and the donor layer thickness on the ejection dynamics during LIFT are lacking. Those parameters are commonly discussed with respect to the minimal achievable droplet size, but therefore often reduced to the generated heat affected volume in the donor layer. Due to the novelty of the presented results on picosecond LIFT, the influence of the pulse duration on the ejection mechanism is not discussed as yet.

To this end, this chapter provides an experimental study on the ejection process related to nanosecond LIFT. To allow for a direct comparison to the results of picosecond LIFT as presented in chapters 5 and 6 , similar experimental conditions specified by the donor layer thickness and the spot size are used in this chapter. First, a brief description of an enhanced experimental imaging setup is presented in section 7.2. Next, fluence resolved image sequences composed of side view images and coaxial view images of the ejection events obtained at various laser fluence levels are discussed. Focusing on the low fluence regime in section 7.3.2, a time-resolved image sequence of low fluence droplet ejections, complemented by high-resolution SEM images of a resolidified ejection event are presented. Then in section 7.4, a discussion on the influence of the laser pulse duration on the ejection mechanism during LIFT, at the ejection threshold is presented. Moreover, the occurrence of thermal radiation and the generation of a laser-induced plasma are briefly addressed. Finally, conclusions are provided in section 7.5.

\subsection{Methods}

Figure 7.1 shows a schematic drawing of the experimental LIFT setup. LIFT experiments were performed using a $6 \mathrm{~ns}$, frequency doubled Nd:YAG laser source emitting a central wavelength of $532 \mathrm{~nm}$. The laser was focused onto the carrierdonor interface using a $50 \times$ long-working distance objective. The laser spot size $\left(1 / \mathrm{e}^{2}\right)$ was measured to be $10 \pm 1 \mu \mathrm{m}$. The laser fluence applied during the experiments are expressed in terms of peak fluence values [117]. Extra-white soda lime glass was used as a carrier substrate. This carrier was precoated with a $200 \mathrm{~nm}$ thick layer of gold, using magnetron sputtering with a sputter rate of $23 \mathrm{~nm} / \mathrm{min}$ as described in section 4.3 . Two $10 \times$ microscope objectives were placed in the LIFT beam path to control the beam divergence and thereby align the focal plane of the LIFT laser beam with the imaging plane of the coaxial imaging setup.

High-resolution images of the LIFT ejection process were captured from two perspectives and at two different time instances. For this purpose, two cameras referred as "dual-shot cameras" that can be triggered to capture two sequential images separated with a time delay of $500 \mathrm{~ns}$, are used. First, side view images 


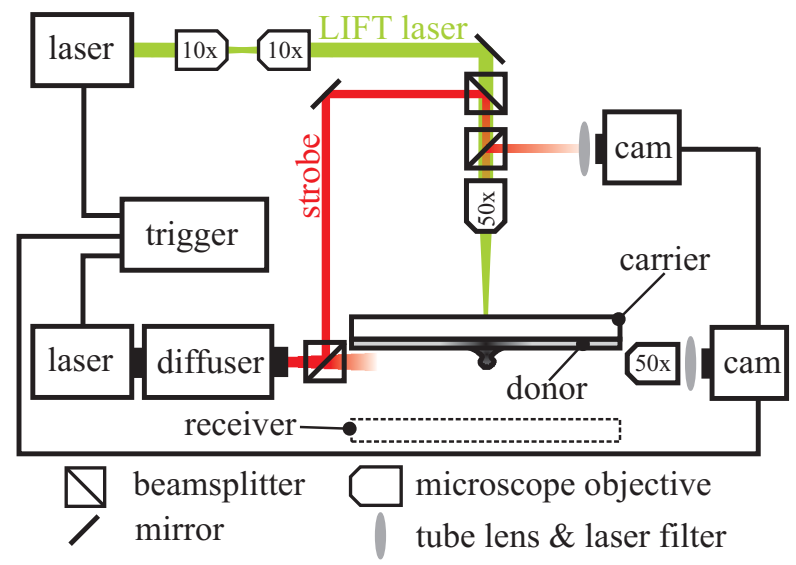

Figure 7.1: Sketch of the experimental LIFT and imaging setup used to study the ejection phenomena in nanosecond LIFT.

were recorded using a combination of such a dual-shot camera and a dual-cavity nanosecond laser source, for strobe illumination. More specifically, a frequency doubled Nd:YAG laser with a pulse duration of $6 \mathrm{~ns}$ and a wavelength of $532 \mathrm{~nm}$ was used as a stroboscopic illumination source. In order to increase the contrast and to avoid interference effects, a high-efficiency fluorescent diffuser was placed in the beam path of the strobe laser beam. The mean wavelength emitted by this diffuser is specified to be $577 \mathrm{~nm}$. The required spatial resolution was achieved by a combination of a $50 \times$ long-working distance objective and a $200 \mathrm{~mm}$ tube lens. To suppress radiation from the LIFT laser source entering the camera, an optical longpass filter was placed in the infinite path of the microscope setup. For the coaxial view of the ejection process, the optical axis of the second dual-shot camera was aligned with the LIFT beam path. The images were obtained using a $50 \times$ long working distance objective, which was also used to co-axially focus the LIFT laser beam onto the carrier-donor interface. Also, this objective was combined with a $200 \mathrm{~mm}$ infinite corrected tube lens, that was mounted to the camera. Any back reflection of the focused LIFT laser beam, was suppressed using an optical longpass filter. To ensure a minimal temporal jitter, the coaxial and the side view cameras share one strobe source. The temporal control of all components was achieved using a BNC pulse delay generator. The temporal jitter of the LIFT laser with respect to the strobe source, was estimated to be less than $10 \mathrm{~ns}$. By combining the dual-shot camera with the dual-cavity strobe illumination source, each ejection event was captured twice (referred to in the following as image A and image B) with a set temporal delay between the images of $500 \mathrm{~ns}$. 

(a) 200
(b) 280
(c) 360
(d) 460
(e) 560
(f) 800
(g) 1080
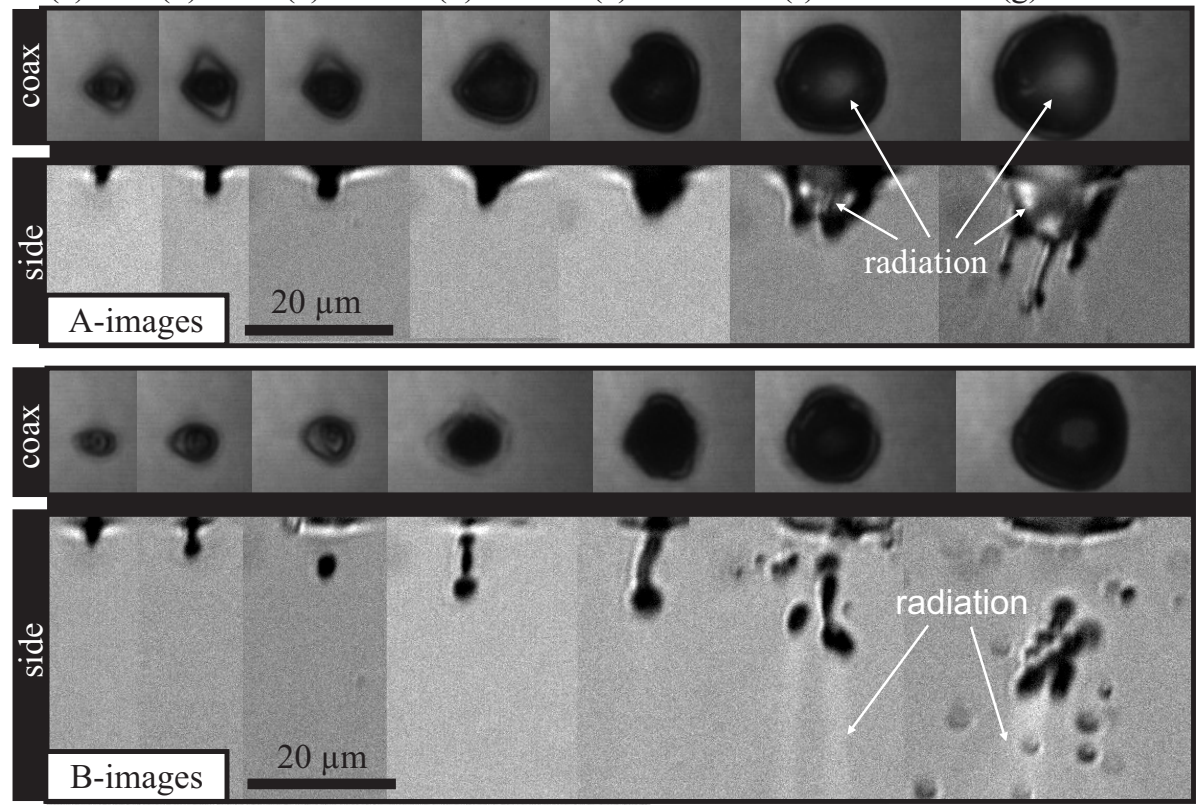

Figure 7.2: $A$ and B images of the coaxial and side-view of the nanosecond LIFT ejection process, that were taken at $300 \mathrm{~ns}$ and $800 \mathrm{~ns}$ after the ejection, respectively. (b) - (e) Laser fluence levels just above the transfer threshold of $280 \mathrm{~mJ} / \mathrm{cm}^{2}$ lead to the formation of a liquid jet, which subsequently contracts into a droplet. (f) - (g) Increasing laser fluences lead to an uncontrolled ejection process, indicated by the formation of a blistering gold bubble.

\subsection{Results}

\subsubsection{Ejection regimes}

Figure 7.2 shows the ejection process obtained at varying laser fluence levels. Starting at a fluence level of $200 \mathrm{~mJ} / \mathrm{cm}^{2}$, which is just below the transfer threshold fluence of $280 \mathrm{~mJ} / \mathrm{cm}^{2}$, the laser fluence was increased up to $1080 \mathrm{~mJ} / \mathrm{cm}^{2}$. Each ejection was captured at two time instances (300 ns and $800 \mathrm{~ns}$ ) after the start of the LIFT laser pulse, indicated by the A- and B-images. Both, coaxial (top) view and side (bottom) view images of the donor layer are shown. Below the threshold fluence of $280 \mathrm{~mJ} / \mathrm{cm}^{2}$ indicated by figures 7.2 (a) and 7.2 (b), no ejection is observed. Instead, a resolidified (frozen) jet, i.e. a non-ejection event was captured. Comparing the A- and the B-images in 7.2 (a) and 7.2 (b) shows a solely deformed jet, which is characterized by a partially contracted jet, resulting into the partial formation of a droplet at the tip of the jet. For slightly higher fluence levels, i.e. figures 7.2 (c) to 7.2 (e), the formation of an initially arbitrary deformed dome can be identified in the A-image. At a later instance (B-images), this ejected dome contracted into a jet like feature, with a contracted droplet at 
the tip. At these fluence levels the ejection process seems to be dominated by the full melting and the resulting stress relaxation of the heated gold layer, similar to what has been reported for femtosecond LIFT of $60 \mathrm{~nm}$ gold layers [84]. Figures 7.2 (f) to 7.2 (g) show the ejection process at higher laser fluence levels. For fluence levels above $800 \mathrm{~mJ} / \mathrm{cm}^{2}$ the formation of a strongly deformed bubble is observed, in the A-images. The rupture of these bubbles, from the donor layer, lead to an uncontrolled ejection process, which is characterized by an ejection of multiple droplets, as can be observed in the B-images.

In addition to the side view images, the coaxial images are shown in figure 7.2. These images indicate the crater diameter increases with increasing laser fluence values, which is related to the Gaussian beam distribution of the focused LIFT laser beam. Comparing the A- and B-images shows that the crater diameter decreases in time, indicating a reflow of material towards the symmetry axis of the ejection crater. This may be caused by surface tension, that contracts the liquid rim (crater edge) towards the center.

Further, in figures 7.2 (f) and 7.2 (g), the emission of broad-band radiation is observed (indicated by white arrows) in both the coaxial view as well as the side-view images. This radiation may originate from laser-induced breakdown, i.e. the formation of plasma or from the emission of thermal radiation. The origin of the observed radiation is discussed in more detail in section 7.4.2.

\subsubsection{Jet ejection}

As the low fluence ejection regime during LIFT, is considered most promising to achieve clean and controlled droplet deposition, this section focuses on the low fluence regime during nanosecond LIFT.

\section{Ejection dynamics}

Figure 7.3 shows the ejection process captured at a laser fluence level of $460 \mathrm{~mJ} / \mathrm{cm}^{2}$. Figure 7.3 (a) shows a typical time- resolved image series that was chosen to give a qualitative impression of the ejection process. Starting at $300 \mathrm{~ns}$ after the laser pulse, the initially flat donor layer deforms into a liquid jet, which is still connected to the donor layer, i.e. to the melt pool that is generated from the absorbed laser energy. At $600 \mathrm{~ns}$, the jet reaches a critical length at which the surface tension leads to an instability of the liquid jet. As a result, a droplet is separated from the jet as observed at 800 ns. At this instance, the ejection speed was measured to be approximately 10 $\mathrm{m} / \mathrm{s}$.

Since marginal differences in the initial conditions, such as film thickness and laser fluence can strongly affect the jet break-up time, the amounts of ejected droplets vary even for a single laser fluence value. Figures 7.3 (b) to 7.3 (d) show that experiments with identical input parameters result in 7.3 (b) clean (59\%), 7.3 (c) multiple (41\%) or 7.3 (d) deflected ejections of either a single or multiple droplets. In contrast to prior observations, these ejections do not propagate perpendicular to the donor layer, but were deflected up to an angle of $\theta=42.5^{\circ}$. Based on the analysis of 100 recorded 
(a)

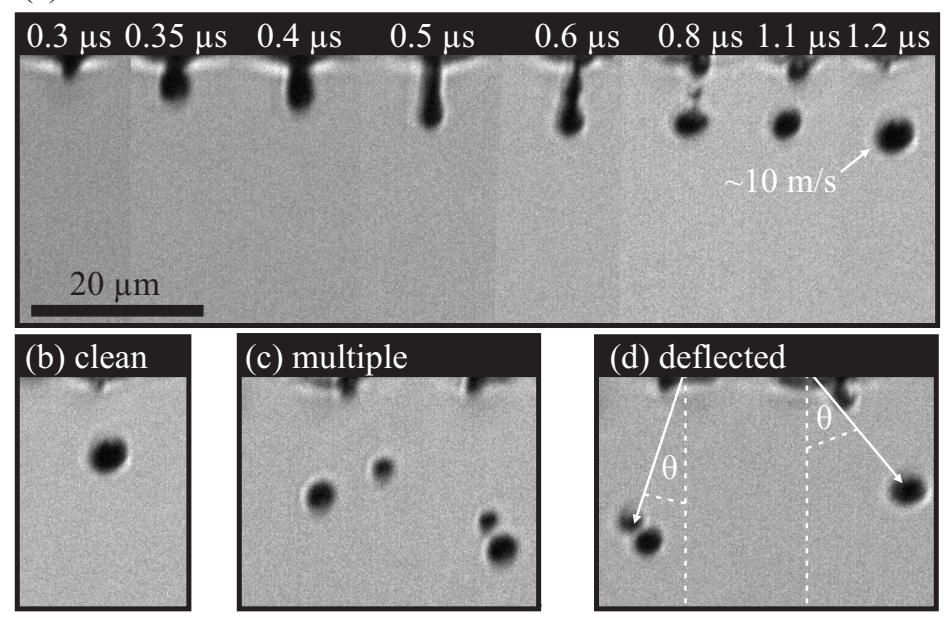

Figure 7.3: Ejection process captured at a laser fluence level of $460 \mathrm{~mJ} / \mathrm{cm}^{2}$. (a) Time sequence of the formation and subsequent contraction of a liquid gold jet into (b) a single droplet, (c) multiple droplets (two ejections shown) and (d) a deflected single (right) or multiple droplets (left). Images (b) to (d) are captured with a time delay of $1.2 \mu$ s with respect to trigger signal of the LIFT laser source.

ejection events, the median angle of deflection was measured to be $11.0^{\circ}$, with a standard deviation of $7.3^{\circ}$. It is assumed that asymmetry of the power density profile of the focused LIFT laser beam and/or irregular thickness of the donor layer has led to this nonzero angle. Although the cause of this deflection is not fully understood yet, the significant range of ejection angles suggests a strong influence on these parameters, and would therefore be interesting to be studied in more detail. The latter is out of the scope of this thesis.

\section{Ejection statistics}

For many applications of LIFT, the exact number of ejected droplets is of major interest and often limited to single droplet ejection. To this end, figure 7.4 shows a statistical overview of the number of ejected droplets as a function of the laser fluence, based on 10 measurements for each fluence value. Increasing the laser fluence results in an increase of the averaged observed droplets, as well as an increase in the measured standard deviation. The percentage values in figure 7.4 refer to the cases in which at least one ejected droplet is observed. Due to the lack of sufficient data (20\%) at the transfer threshold, the observation at $280 \mathrm{~mJ} / \mathrm{cm}^{2}$ does not show an error bar. However, it is clear that "clean", i.e. single droplet ejection may be expected only for fluence values just above the threshold, as an increased fluence level increases the number of ejected droplets. 


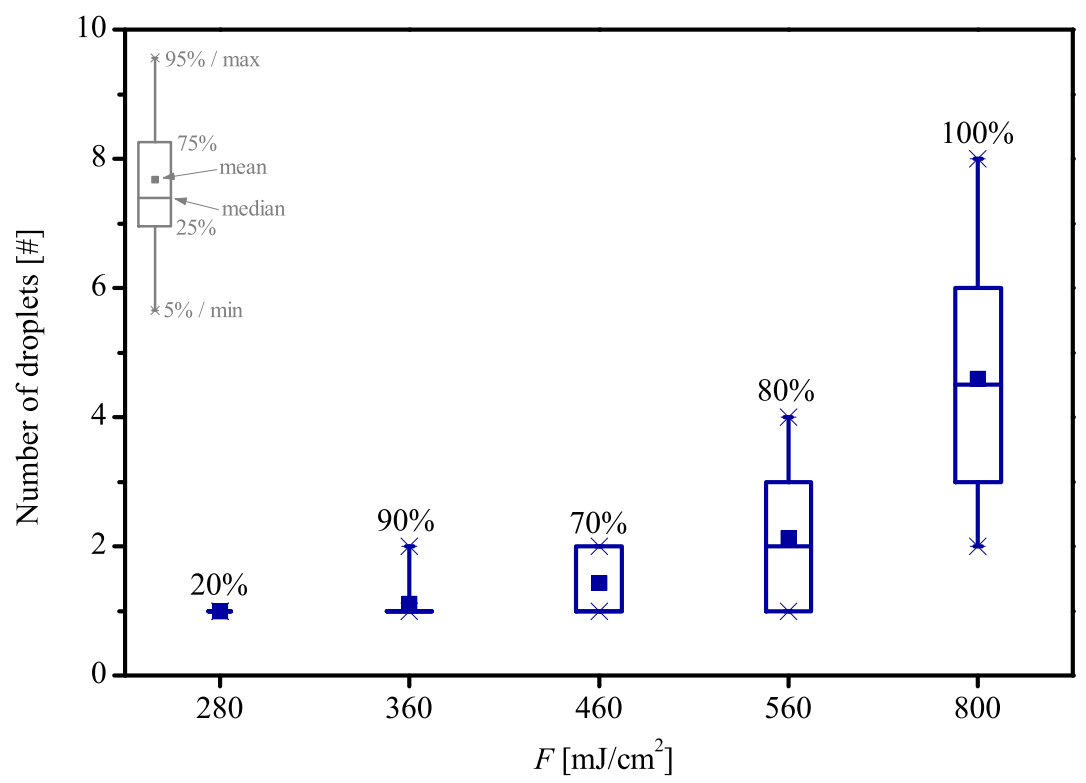

Figure 7.4: Observed number of droplets as a function of the laser fluence in nanosecond LIFT. The percentage values refer to the cases in which at least one ejected droplet is observed. The statistics are based on 10 measurements for each fluence value.

\section{Donor layer conditions}

The observed ejection dynamics as shown in figure 7.3 indicate an ejection mechanism that is dominated by the hydrodynamics of the laser generated melt pool in the donor layer. In literature, similar observations are discussed and related to the inertia driven jet formation at the apex of an emerging liquid gold bubble [42]. However, those studies employed different experimental conditions, since those studies are based on femtosecond laser pulses and a donor layer thickness of only $60 \mathrm{~nm}$. The reasoning of the authors is based on the formation of a hollow zero pressure bubble, which was found to be key for the proposed ejection process. As the presented results in this chapter are obtained with nanosecond pulses and a donor layer thickness of $200 \mathrm{~nm}$, a detailed investigation on the donor layer is presented in order to investigate whether a similar ejection mechanism can be considered to explain the observed jet formation at the transfer fluence threshold.

Figure 7.5 (a) shows a SEM image of a "frozen" ejection event obtained at a laser fluence level of $200 \mathrm{~mJ} / \mathrm{cm}^{2}$, corresponding to a fluence level slightly below the transfer threshold. A frozen jet located at the apex of a frozen bubble is observed. The bubble shows strong indications of crystal structures, attributed to the resolidification process at a rather slow cooling rate. The width of the resolidified jet is estimated to be $\approx 1 \mu \mathrm{m}$. Figure 7.5 (b) shows several cross-sections at different locations of the frozen ejection event, at locations as indicated in figure 7.5 (a). In figure 7.5 (b) 


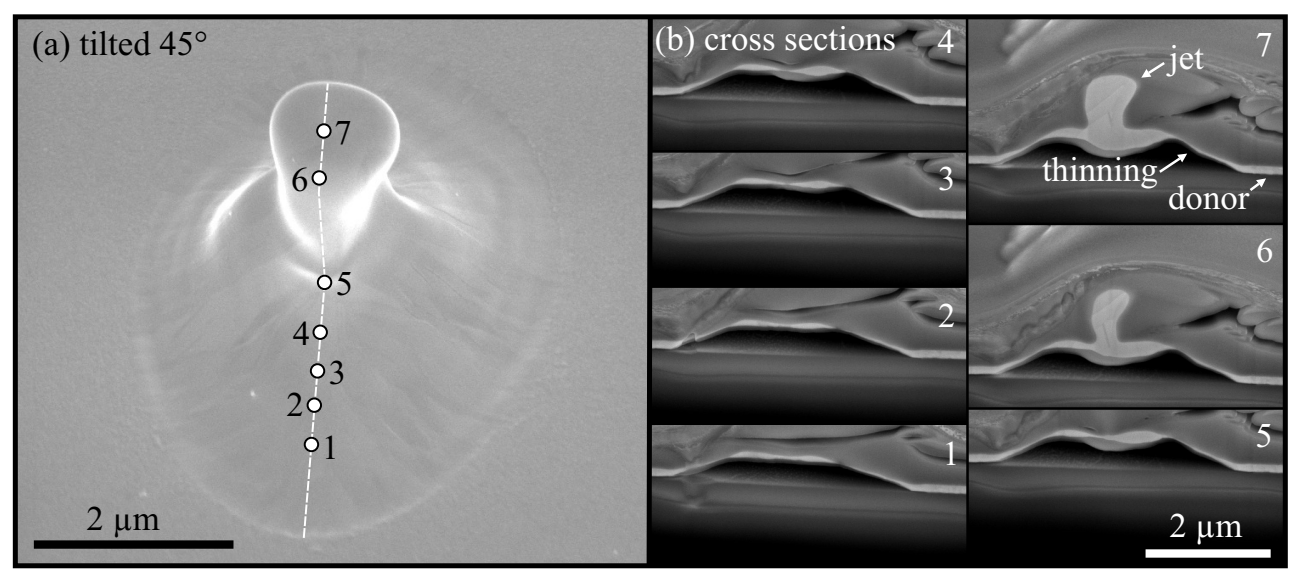

Figure 7.5: SEM images of a frozen ejection event due to a nanosecond laser pulse, obtained at laser fluence of $200 \mathrm{~mJ} / \mathrm{cm}^{2}$. Figure (a) shows an isometric view of the frozen jet that is located at the apex of a resolidified bubble, due to relaxation and hydrodynamics of the molten gold layer. (b) Shows several cross-section images at different locations of the frozen ejection event, as indicated in (a).

several SEM images starting at the outer area of the bubble towards the apex and the jet are shown. From these images three conclusions are drawn as indicated in figure 7.5 (b) insert 7. First, the bubble is obviously hollow. Second, a thinning of the bubble is observed in the transition from the outer area towards the apex. Third, a jet is located at the apex of the bubble. However, a second jet facing from the apex towards the carrier, referred to as a "counter-jet" $[42,84]$, is not identified. Despite the absence of the counter-jet, a significant growth and thickening of the apex is observed. For increasing laser fluence levels the formation of the reported counter-jet is likely, as the increase in laser fluence will also lead to an increased inertia towards the apex, which may ultimately trigger the formation of such a counter-jet.

\section{Driving mechanism of jet ejection}

Based on the observations made during low fluence nanosecond LIFT, it assumed that a similar mechanism, as proposed by Unger et al.[84] can be employed in order to explain the observed ejection mechanism during nanosecond laser-induced forward transfer. A brief description of the stages of this ejection mechanism is illustrated in figure 7.6. First, the incident laser energy is absorbed within the optical penetration depth of the donor layer. Due to the rather long laser pulse duration of $6 \mathrm{~ns}$, common heat diffusion occurs. The established temperature gradient is dominated by the geometrical shape of the Gaussian profile which leads to a temperature gradient perpendicular to the optical axis, see figure 7.6 (a). Next, stress relaxation leads to the deformation of the donor layer. 7.6 (b) Due to the boundaries given by the carrier and the surrounding donor material, the donor layer starts to buckle and a bubble emerges towards the air interface. 7.6 (c) The 


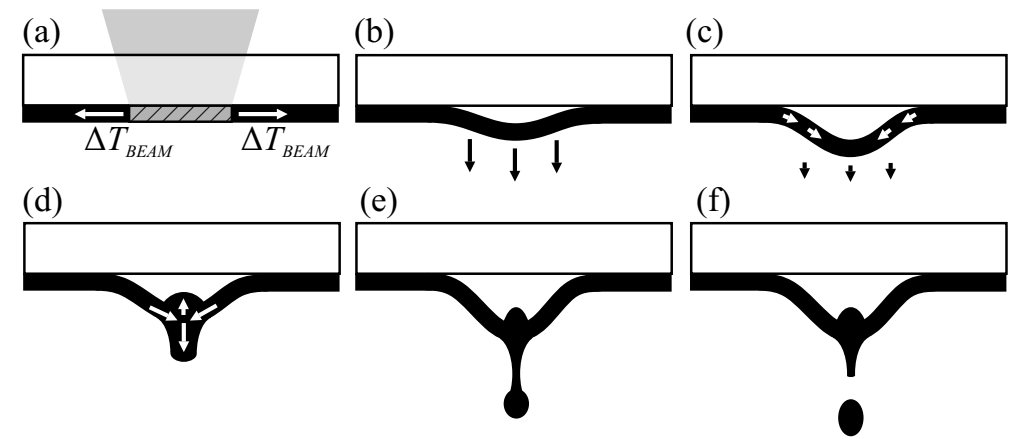

Figure 7.6: Illustration of the stages of the ejection mechanism during nanosecond LIFT, at the transfer threshold. (a) Laser energy is absorbed in the donor layer and the driving temperature gradient establishes. (b) Bubble expands due to stress relaxation. (c) Low pressure in the captivated bubble hampers emerging bubble. (d) Liquid flow towards the apex due to the initial inertia. (e) Jet is formed at the apex of the emerged bubble. (f) Jet separates into droplets due to strong surface tension forces.

increasing size of the bubble leads to a reduced pressure in the interior of the bubble, which hampers the speed of the emerging bubble. Due to the inertia of the liquid film this deceleration of the bubble leads to liquid motion within the film towards the apex of the bubble. 7.6 (d) Due to the conservation of inertia, the liquid flow towards the apex of the bubble initiates the formation of the jet. Finally, the jet elongates 7.6 (e) and subsequently separates into a single or multiple droplets due to strong surface tension forces [42], as illustrated in figure 7.6 (f).

\subsection{Discussion}

\subsubsection{Effect of the pulse duration on the ejection mechanism}

In the following, a discussion on the effect of the laser pulse duration on the LIFT process is provided. Therefore, the reasoning focuses on the ejection dynamics during picosecond and nanosecond LIFT, obtained at laser fluence levels corresponding to the low fluence regime.

As was shown in chapter 6 , the low fluence ejection regime during picosecond LIFT is found to be characterized by the ejection of a cap like film, which is supposed to be directly correlated to the geometrical shape of the Gaussian laser pulse. Moreover, the generation of a transversal stress wave within the donor layer was proposed as a reasonable explanation.

The presented results on the low fluence ejection regime during nanosecond LIFT indicate a completely different ejection mechanism. It was shown in section 7.3.2, that the ejection mechanism is related to the stress relaxation of the donor layer which ultimately leads to the formation of liquid jet, located at the apex of a liquid bubble. The ejection process is explained on the basis of the hydrodynamics 

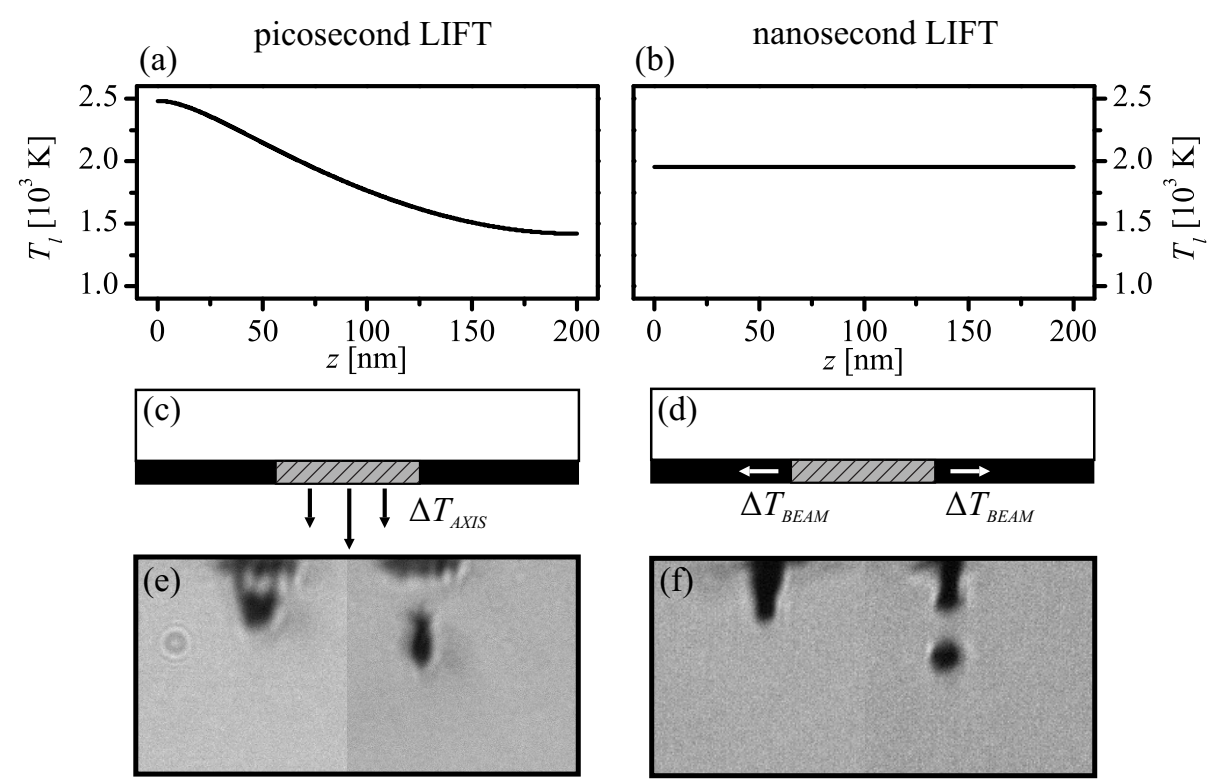

Figure 7.7: Comparison of picosecond and nanosecond driven LIFT at the low fluence regime. (a) and (b) show the computed temperature distribution along the thickness of the donor layer at a laser fluence of $360 \mathrm{~mJ} / \mathrm{cm}^{2}$. (c) and (d) show an illustration of the dominating temperature gradients that are established in the donor layer. (e) and (f) show images of the observed ejection events as shown in sections 6.3.1 and 7.3.2.

of the emerging liquid metal bubble.

Figure 7.7 shows the computed temperature distributions across the donor layer for similar laser fluence values, but different pulse durations of 6.7 ps and $6 \mathrm{~ns}$, respectively. To this end, the TTM as described in section 3.3 was extended to a $2 \mathrm{D}$ axis-symmetric simulation. The temperature distribution shown at $2 \tau_{p}$ after the laser pulse represents the temperature distribution after the full laser pulse is absorbed by the donor layer. Of course, for later instances thermal diffusion takes place, and the distribution across the donor layer "smoothens". However, the initial temperature gradient is of relevance, as it triggers the ejection process and provides the initial inertia to the ejection process. In the picosecond regime, a strong gradient is observed. For nanosecond pulses a nearly homogeneous distribution occurs.

In order to understand the difference between nanosecond and picosecond LIFT, the generated temperature gradient in the donor layer is of major relevance. Figure 7.7 (a) shows the calculated temperature distribution for picosecond LIFT. The temperature decays along the $z$ axis and a temperature gradient of approximately $\Delta T_{A X I S} \approx 5000 \mathrm{~K} \mathrm{\mu m}^{-1}(1000 \mathrm{~K} / 200 \mathrm{~nm})$ is established. In comparison, the gradient given by the geometrical shape of the Gaussian profile is estimated to be $\Delta T_{B E A M} \approx 250 \mathrm{~K} \mathrm{\mu m}^{-1}(2500 \mathrm{~K} / 10 \mu \mathrm{m})$. Hence, the overall temperature gradient is dominated by the temperature gradient $\Delta T_{A X I S}$ across the donor layer and the 


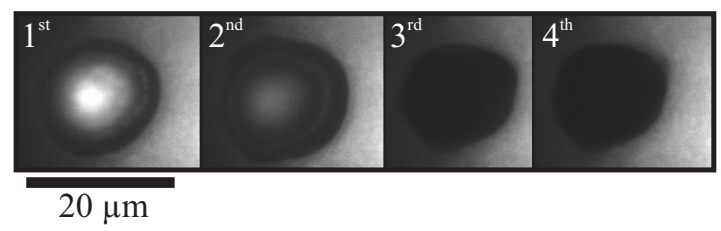

Figure 7.8: Coaxial view on the donor layer, which is subsequently exposed to four laser pulses. The bright radiation which can be observed at the first and second image disappears completely after the second laser pulse.

effect of the beam profile can be neglected. As a consequence also the generated stress wave is dominated by the transversal stress wave, propagating in the layer, which leads to a local dependency of the generated stress on the beam profile, see figure 7.7 (c). Finally, the donor layer is ejected in a cap like shape, see figure $7.7(\mathrm{e})$.

For nanosecond laser pulses, heating of the donor layer leads to a uniform temperature distribution across the layer, see figure 7.7 (b). Hence, the overall temperature gradient is dominated by the geometrical shape of the Gaussian profile, which was estimated to be $\Delta T_{B E A M} \approx 50 \mathrm{~K} \mathrm{\mu m}^{-1}$. This temperature gradient is established perpendicular to the optical axis, see figure $7.7(\mathrm{~d})$. The stress relaxation leads to buckling of the donor layer, which ultimately causes the formation of the jet, see figure 7.7 (f).

The driving forces during picosecond and nanosecond LIFT are of a different scale, as the temperature gradient, and therefore also the generated stress, in the donor layer are of a different order of magnitude. This may explain the different ejection velocities, since cap ejections were measured with velocities ranging from 30 $\mathrm{m} / \mathrm{s}$ to $100 \mathrm{~m} / \mathrm{s}$, whereas nanosecond ejections were found to travel at velocities in the order of $10 \mathrm{~m} / \mathrm{s}$. Based on the presented results, a direct relation between the observed ejection mechanism and the generated temperature gradient in the donor layer can be derived. Exploiting this relation allows to select on forehand the proper layer thickness for a given laser pulse duration and a desired ejection mechanism, i.e. cap or jet driven ejection.

\subsubsection{Radiation}

Figure 7.2 (f) and 7.2 (g) show broad band radiation (bright areas) indicated by arrows, observed both in the side view and coaxial view images of the ejection process. In this subsection, possible sources of radiation are investigated. To this end, the origin and the type of radiation, i.e. plasma radiation or thermal emission due to the elevated temperatures of the donor layer, are discussed.

\section{Origin of the radiation}

In the following, a first interpretation of the origin of the observed radiation is discussed. First of all, it needs to be discussed, whether the observed light is related 
to the heating of the gold layer or originates from the carrier material (soda-lime glass). To that end, figure 7.8 shows a series of coaxial images that were obtained after exposing the donor to four laser pulses at a fixed laser fluence, and captured at a fixed time-delay of $10 \mathrm{~ns}$ after the LIFT laser pulse. Each image shows the same ejection crater in the donor layer, after every subsequent laser pulse. The bright radiation can be clearly observed due to the first and second laser pulse, but not due to the third and fourth. That is, after the second laser pulse only the prior formed crater in the donor layer is visible. As the radiation is not persistent over multiple laser pulses, it is concluded that the source of radiation cannot originate from the carrier material, but is correlated to the gold donor layer.

\section{Laser-induced plasma formation}

In order to explain the source of these observed emissions, the formation of a laserinduced plasma is considered. As concluded above, plasma formation is expected only for vaporized gold and not for evaporated carrier material. Here, volumetric heating across the donor layer is assumed. This is a valid assumption, as the thermal penetration depth $\delta_{D}$

$$
\delta_{D}=2 \sqrt{D \tau_{D}}
$$

with the thermal diffusivity $D=12.8 \cdot 10^{-5} \mathrm{~m}^{2} \mathrm{~s}^{-1}$ and the diffusion time scale $\tau_{D}=6$ ns, exceeds the donor layer thickness by one order of magnitude. Hence, the fluence level $F_{p l}$ to induce a laser induced plasma is at least the fluence level that is needed to fully vaporize the donor layer. This threshold fluence can be estimated from

$$
F_{p l}=d \frac{C_{p} \Delta T+\rho\left(L_{m}+L_{v}\right)}{1-R}
$$

with the temperature increase to the vapor temperature $\Delta T=2829 \mathrm{~K}$, the density $\rho=19300 \mathrm{~kg} \mathrm{~m}^{-3}$, the latent heat of melting $L_{m}=0.6 \cdot 10^{5} \mathrm{~J} \mathrm{~kg}^{-1}$, the latent heat of vaporization $L_{v}=1.6 \cdot 10^{6} \mathrm{~J} \mathrm{~kg}^{-1}$, the heat capacity $C_{p}=2.48 \cdot 10^{6} \mathrm{~J} \mathrm{~m}^{-3} \mathrm{~K}^{-1}$ and the reflection coefficient $R=0.64$. The plasma threshold fluence $F_{p l}$ is calculated to be $2178 \mathrm{~mJ} / \mathrm{cm}^{2}$. In contrast, the observed radiation in figure 7.2 is detected at fluence levels, well below the calculated threshold of vaporization. Hence, the formation of a laser-induced plasma appears to be unlikely and is therefore discarded to explain the observed emissions.

\section{Thermal emission}

A second cause of the observed emissions may be found in the thermal emission (Planck emission) due to the elevated temperature of the heated gold layer and the ejected droplets. The emitted spectral irradiance $I_{\lambda}$ as a function of the emission wavelength $\lambda$ and the gold temperature $T_{l}$ is described by Planck's equation as

$$
I_{\lambda}\left(\lambda, T_{l}\right)=\frac{2 \pi h c^{2}}{\lambda^{5}\left(\exp \left(h c /\left(\lambda k_{B} T_{l}\right)\right)-1\right)},
$$




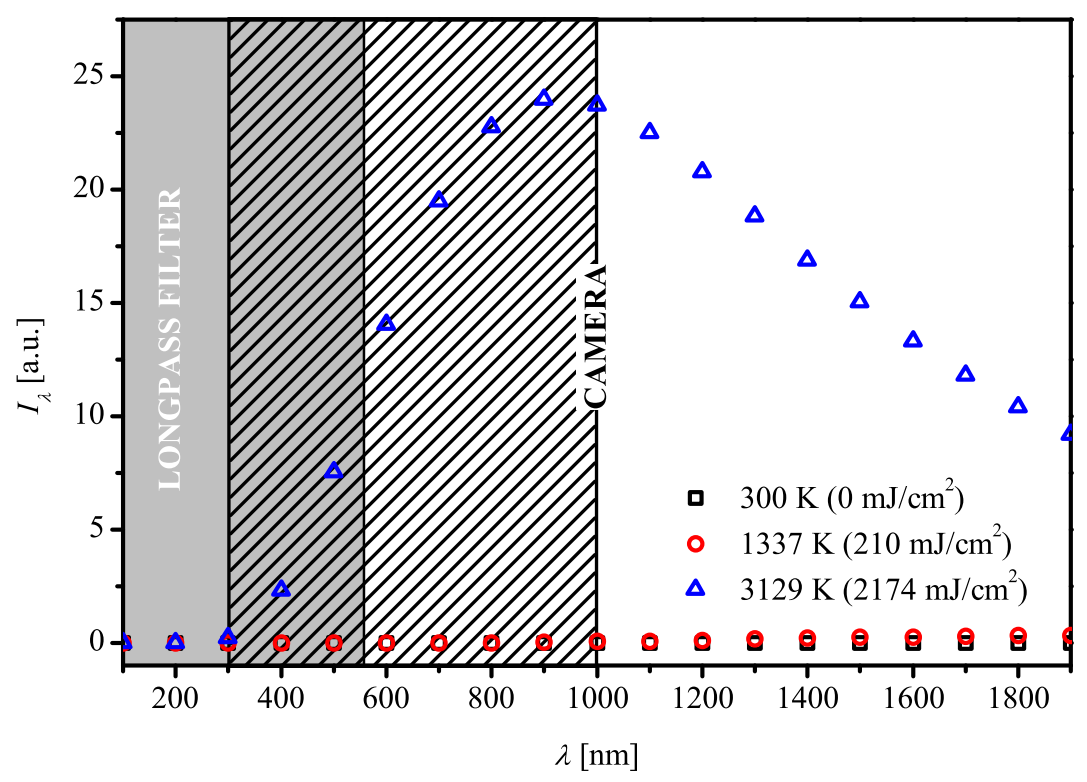

Figure 7.9: Spectral irradiance calculated from equation (7.3) for three temperatures $(300 \mathrm{~K}, 1337 \mathrm{~K}$ and $3129 \mathrm{~K})$ as a function of the wavelength. The gray area indicates the spectrum blocked by the optical longpass filter that was used to supress the LIFT laser radiation. The hashed area indicates the spectral sensitivity range of the camera used.

with the Planck constant $h=4.14 \cdot 10^{-15} \mathrm{eV} \mathrm{s}$, the speed of light $c=3 \cdot 10^{8} \mathrm{~m} \mathrm{~s}^{-1}$ and the Boltzmann constant $k_{B}=8.62 \cdot 10^{-5} \mathrm{eV} \mathrm{K}^{-1}$.

Figure 7.9 shows the corresponding calculated spectral irradiance (markers) as a function of the emission wavelength, at three temperatures. The temperatures of $300 \mathrm{~K}, 1337 \mathrm{~K}$ and $3129 \mathrm{~K}$ correspond to the room-, melting and vaporization temperature of gold, respectively. The corresponding fluence values were obtained by equation (7.2). The range of the spectral sensitivity of the camera (about 300 $\mathrm{nm}$ to $1000 \mathrm{~nm}$ ) is indicated by the hashed area in figure 7.9. The longpass laser filter that was used to block the LIFT laser radiation is indicated by the gray area (about $100 \mathrm{~nm}$ to $550 \mathrm{~nm}$ ). As can be observed, heating of the gold above the melting temperature results in a significant increased Planck emission within the sensitive spectral range of the camera, which may explain the observed emissions. This conclusion is supported by additional analysis of side view images, which are presented in the following.

Figure 7.10 (a) shows a timing diagram that indicates the integration time - i.e. time period in which the camera shutters are open - as well as the duration of the strobe laser pulses. This scheme is important to understand the observed emissions. As indicated in figure 7.10 (a), the strobe pulse was triggered at the end of the shutter-open-period, leading to image A and the start of the shutter-open-period, leading to image B. Figures 7.10 (b) and 7.10 (c) show the ejection of a droplet in 


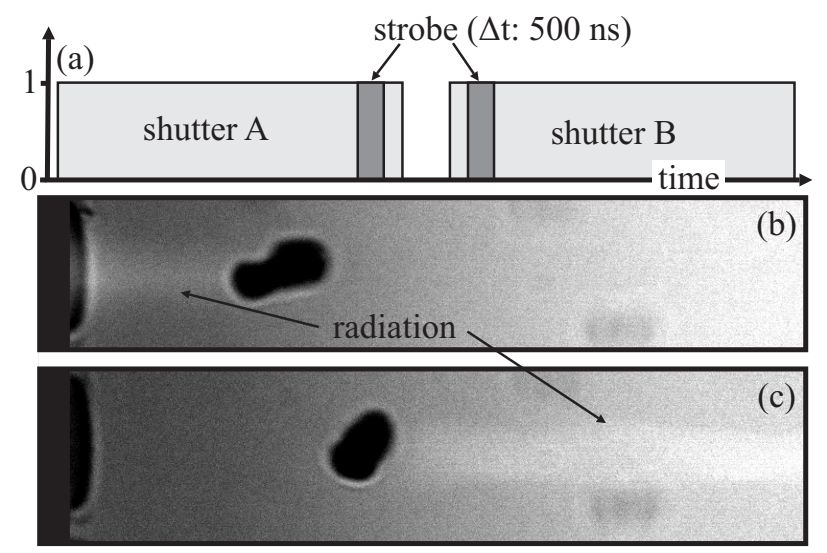

Figure 7.10: (a) Sketch of the timing sequence of the camera shutter with respect to the strobe pulses. (b)-(c) Show the donor layer on the left and the ejected droplet propagating from left to right. The ejected droplet was captured at two time instances (A- and B-images). The orientation of the observed bright phenomena indicates the presence of Planck radiation.

this time scheme. Both images indicate a bright trace of light, which is located either at the trailing (A image) or the leading edge (B-image) of the ejected droplet. As already discussed, it is assumed that the heated droplet continuously emits Planck radiation, which is captured by the camera as long as the camera shutter is open. Therefore, the observed trace of bright light is the result of the trajectory of the ejected droplet. Here, the waviness of the trace of light in figure 7.10 (c), is proposed to be correlated to the ongoing deformation of the droplet during its trajectory.

At this point it is concluded, that at least the observed radiation in the side view images is attributed to the Planck emission of the heated droplet. The origin of the radiation in the coaxial images is not clarified conclusively. That is, additional measurements such as a spectral analysis of the emission spectrum is needed to definitely determine the origin of the radiation as it allows to discriminate between a Planck and a plasma related source of radiation.

\subsection{Summary}

An experimental study on the ejection regimes of nanosecond LIFT was presented. Two ejection regimes were identified. For laser fluence values just above the transfer threshold of $280 \mathrm{~mJ} / \mathrm{cm}^{2}$, the formation of a liquid gold jet and the subsequent formation and ejection of single and multiple droplets were found. Increasing the laser fluence increases the number of ejected droplets. For laser fluence levels above $800 \mathrm{~mJ} / \mathrm{cm}^{2}$, the formation and rupture of a blistering bubble was observed. This second regime is less suitable for controlled deposition, since multiple droplets are always ejected at angles that cannot be controlled as yet. A description of the 
low fluence jet ejection regime was provided and compared to the observed cap ejection during picosecond LIFT. The different ejection mechanism as observed during picosecond (chapters 5 and 6) and nanosecond LIFT (section 7.3.2) were found to be directly related to the generated stress distribution in the donor layer, and hence to the generated temperature gradient in the latter. Last, the origin of the observed radiation during the ejection process was discussed. Two scenarios related to plasma- and Planck emission have been discussed and were applied to clarify the origin of the radiation in the side view images. The radiation in the coaxial view has not been clarified conclusively, but has been discussed with respect to both potential sources of radiation. 



\section{Chapter 8}

\section{Towards 3D printing}

In this chapter* the deposition process during picosecond LIFT is investigated and demonstrated by the manufacturing of high-aspect ratio pillars. The material properties of those pillars are assessed and discussed with respect to porosity, electrical, and mechanical properties. Besides, the drop impact dynamics are addressed with respect to the drop shape after solidification.

\subsection{Introduction}

Due to continuous progress in additive manufacturing technologies the fabrication of complex 3D structures [118] is routinely achieved. In particular the printing concept has gained momentum for rapid prototyping, rapid manufacturing and rapid tooling since it provides for fast, low-cost, and contact-free deposition at room conditions and poses minimal disturbances to the receiver substrate, on which the material is deposited. However, printing of metals has been limited to low-melting point metals $[4,5,119]$, pastes [120], and metal-containing inks [6-8]. These materials are generally not optimized in terms of material properties, e.g. mechanical strength, electrical conductivity, corrosion rate, and material cost, or suffer from limited resolution [121]. Therefore, fabrication of metal microstructures including micro-antennas [9], out-of-plane electrodes [122], and filling of TSVs for connecting stacked 2D electronic circuits [10] remain ongoing challenges.

Unfortunately, 3D printing using LIFT has not yet been demonstrated, perhaps since at least two challenging requirements have to be simultaneously fulfilled. First, good adhesion between the deposited, i.e. stacked droplets is required, but the deposited droplets generally solidify in a spherical or torus shape [10, 18, 42, 49].

*Parts of this chapter have been published in Advanced Materials, published online, 2015, Claas Willem Visser, Ralph Pohl, Chao Sun, Bert Huis in 't Veld, Gert-Willem Römer, and Detlef Lohse. Towards 3D Printing of Pure Metals by Laser-Induced Forward Transfer. 

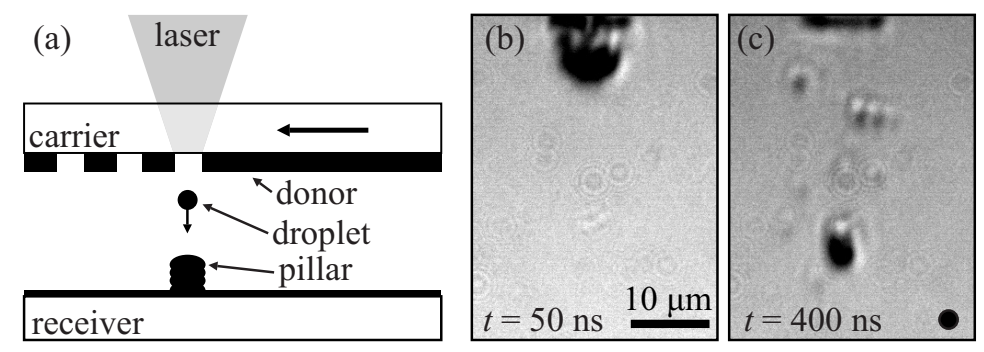

Figure 8.1: (a) LIFT setup in which a laser pulse is focused onto a $200 \mathrm{~nm}$ thick copper donor layer, resulting in the ejection of a liquid copper droplet. By moving the glass carrier in the horizontal plane (shown by the bold arrow), repetitive donor ejections are deposited at the same location on the receiver. The subsequently impacting droplets solidify on top of each other, resulting in the formation of a pillar on the receiver substrate. The receiver is coated with a copper film, to improve the adhesion of the first droplets. Figures (b) and (c) show a single LIFT ejection obtained at a laser fluence of $F=460 \pm 20 \mathrm{~mJ} / \mathrm{cm}^{2}, 50$ ns and $400 \mathrm{~ns}$ after the laser pulse, respectively. The black area in the very top of these images shows the crater. In figure (b), the deformed donor film is just detaching from the donor layer. Figure (c) shows the flying droplet, which is trailed by several smaller satellite droplets. The droplet appears to be much smaller than in figure (a), but as surface tension contracts the initially hollow droplet, it is confined into a sphere with a final size as indicated by the black dot. Eventually, the droplet will reach a spherical shape and impact on the receiver substrate (not shown).

This unavoidably results in porosity and limited drop-to-drop contact when the droplets are stacked on top of each other. A second requirement is that the landing position of a single droplet has to be limited to the previously deposited droplets impact area. This is non-trivial for the relatively large donor-receiver distances required for $3 \mathrm{D}$ printing [48], but was achieved nonetheless for a narrow range of fluence levels.

This chapter presents a concise outlook on fabrication of 3D metal structures using picosecond LIFT and provides first results on the generation of pure-metal pillars by stacking $\mu \mathrm{m}$-sized copper and gold droplets. First, the morphological and physical features of the pillars are assessed in detail. These results are then extended to three printing applications - i.e. the fabrication of extremely high aspect-ratio pillars, the filling of high-aspect ratio TSVs, and the deposition of an electrically conductive line. Second, the relationships between the laser fluence, the droplet impact velocity, and the droplet shape after solidification are discussed, since these aspects are essential for controlled deposition, but have received scarce attention in literature.

\subsection{Methods}

Figure 8.1 (a) shows the setup used for LIFT in this chapter. In short, a laser beam with a pulse duration of $6.7 \mathrm{ps}$ and a wavelength of $515 \mathrm{~nm}$ is focused into a spot with 


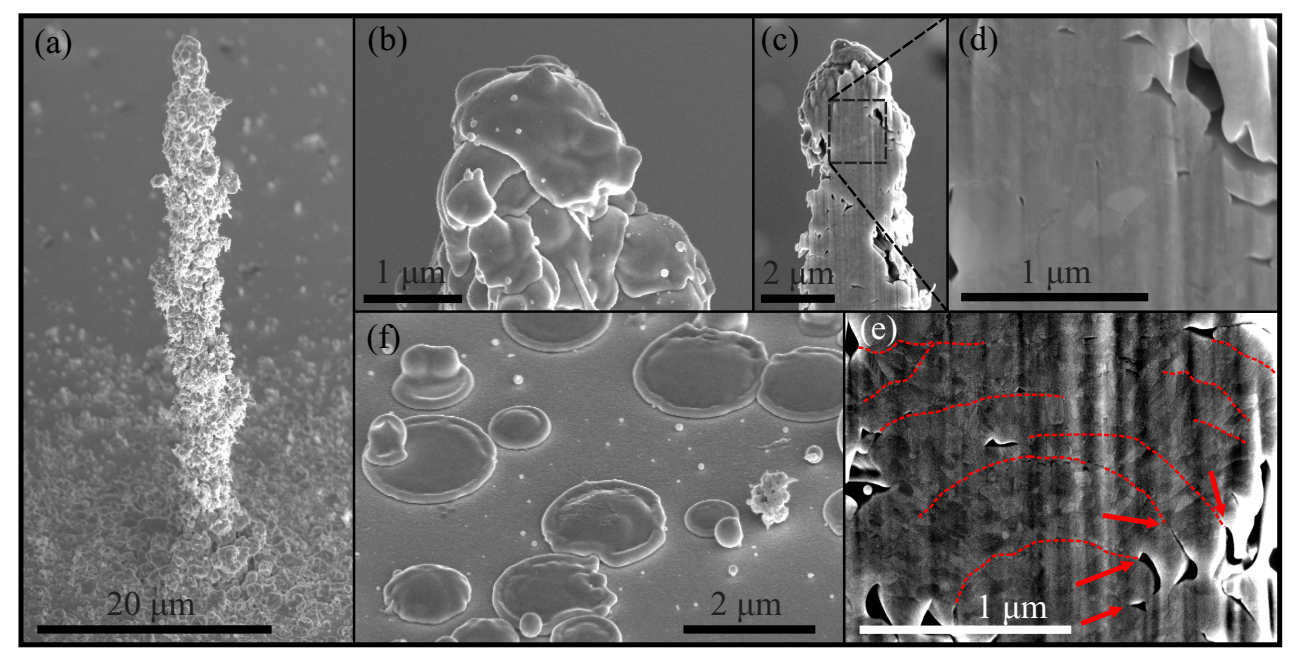

Figure 8.2: SEM images of a LIFT printed copper pillar. (a) Isometric overview image. Although the surface appears rough, the thickness of the pillar is relatively homogeneous. (b) Pillar top, clearly showing the final droplet which spread over the top prior to solidifying. The nano-droplets visible on top of this resolidified droplet originate from satellite droplets caused during the ejection process, see chapter 6. (c) Cross-section of the pillar top, obtained by cutting with a focused ion beam; the region in the rectangle is magnified in figure (d) showing that the interior of the pillar is almost pore-free. (e) Detail of pillar cross-section, showing different grain orientations in different gray levels. Grain boundaries with a length spanning multiple grains are indicated in red. These lines typically end at pores in the edge of the pillar, which correspond to the original droplet edges. Therefore, these long grain boundaries likely correspond the original droplet surfaces. (f) Example view of solidified droplets deposited next to the base of a pillar. Figures (a), (c), and (d) are from the same pillar, whereas the other images are from different pillars processed using the same ejection parameters.

a $1 / \mathrm{e}^{2}$ radius of $8.3 \pm 0.6 \mu \mathrm{m}$, resulting in the melting and ejection of a $200 \mathrm{~nm}$ copper or gold film. See chapter 4 for more details on the experimental setup. As shown in figures 8.1 (b) and 8.1 (c), the ejected material initially maintains a cap-like shape, but strongly contracts to eventually obtain a spherical shape. The droplet ejection velocity [123] ranges from 30 to $200 \mathrm{~m} / \mathrm{s}$, as was discussed in detail in chapter 5 . The donor substrate is moved in the horizontal plane using a motorized translation stage. Repeated laser pulses then result in a series of ejection events at the same spatial position, which leads to the formation of a pillar if a receiver substrate is located below the donor. 


\subsection{Results}

\subsubsection{Microstructure of printed pillars}

To date, the copper pillar shown in figure 8.2 (a) is the first example of high aspect ratio 3D metal micro-manufacturing using LIFT. The pillar has a height of about $80 \mu \mathrm{m}$ and a diameter of $5.3 \pm 0.7 \mu \mathrm{m}$, hence, it exhibits an aspect ratio of about 15 . The pillar top is magnified in figure $8.2(\mathrm{~b})$, showing the last-deposited droplet which has spread out over the pillar top and subsequently solidified. Below this droplet, the edges of several solidified droplets can be observed. To assess whether such round edges result in pores within the pillar, a cross-section of pillar was prepared using a focused ion beam, and subsequently imaged using a scanning electron microscope. As shown in figures 8.2 (c) and 8.2 (d), the original droplets are hardly visible and only a few small pores are observed. Using a through-lens detector in the SEM, the grains within the pillar were visualized. Grain sizes between $50 \mathrm{~nm}$ and $200 \mathrm{~nm}$ are typically observed and seem to constitute most of the pillar volume. Furthermore, several long, horizontally-aligned grain boundaries are identified, as illustrated by the dashed lines in figure 8.2 (e). At the outer surface of the pillar, the ends of these boundaries frequently coincide with voids (pores) corresponding to the edge of solidified droplets. This suggests that the impacting droplets do not melt the pillar surface during impact, but instead crystallize onto the previously deposited droplets. Therefore, these horizontal interfaces could be weak or brittle. This aspect will be accessed in section 8.3.3.

\subsubsection{Repeatability of the printing process}

Images of the deposition process of a $270 \mu \mathrm{m}$ high pillar are shown in figure 8.3 (a). At pillar heights of about $70 \mu \mathrm{m}$ and higher, the growth rate per deposited droplet becomes constant as indicated by the inclined line in figure 8.3 (a). Then the pillar diameter is almost homogeneous. For the first number of droplets the pillar growth rate is nonlinear, due to deviations in the ejection angle of the droplet resulting in a spread of the impact location. Therefore, the bottom $30 \mu \mathrm{m}$ of the pillar is slightly thicker than the rest of the pillar. This thickening is easily prevented by reducing the donor-receiver distance by $\approx 50 \mu \mathrm{m}$, but could be beneficial if a strong pillar-receiver adhesion is required. The reproducibility of the pillars is illustrated in figure 8.3 (b), showing five pillars, which were deposited by moving the receiver and repeating a series of ejection events with identical laser parameters.

\subsubsection{Mechanical and electrical properties}

The mechanical and electrical properties of the pillars and their thickness were studied. The mechnical properties were assessed by deflecting beam measurements, as shown in figure 8.4 (a). To determine the pillar radius $r_{p i}$, atomic force microscopy measurements were performed yielding the deflection $\delta_{p i}$ of a beam as a function of the force $F_{A F M}$, as shown in figure 8.4 (a). After each measurement, the pillar was allowed to relax to its original position to verify that no plastic deformation of the 

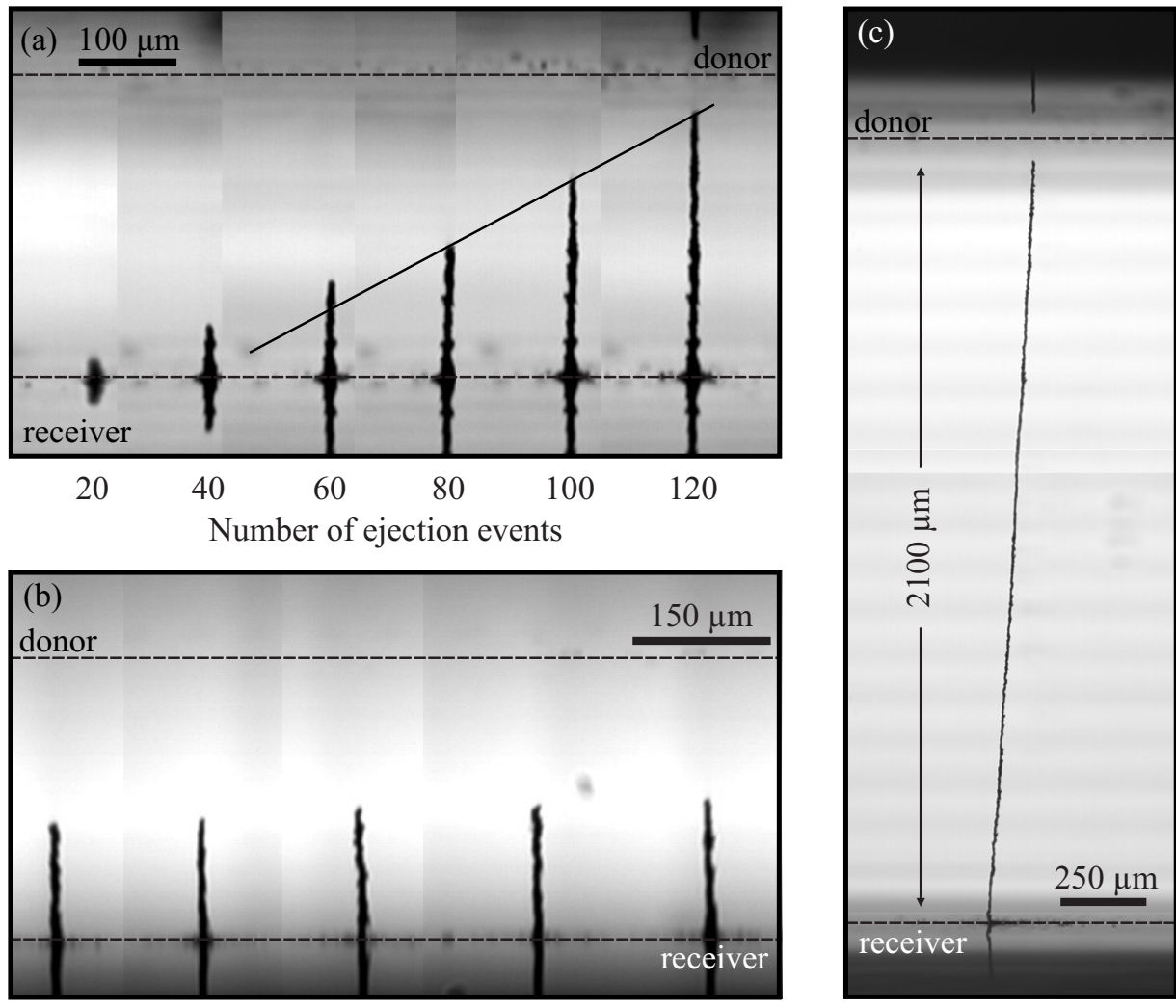

Figure 8.3: (a) Optical microscopy images of copper pillars after a number of deposited droplets. The solid line indicates a constant pillar growth rate of $2.3 \mu \mathrm{m} /$ droplet, which is observed if the pillar tip is sufficiently close to the donor surface and all droplets land on the tip. (b) Images of five LIFT printed copper pillars on the same receiver, while using identical process parameters. The dashed lines indicate the (reflective) donor and receiver surfaces. (c) Gold pillar with a height of $2100 \mu \mathrm{m}$, illustrating the potential for fabrication of free-standing structures.

pillar occurred. The effective thickness of the pillar is calculated to be $0.9 \pm 0.15 \mu \mathrm{m}$. This deflection is plotted as a function of the force in figure 8.4 (b). As can be observed, the deflection increases linearly with the force, as is expected for elastic deformation, and the curves indicate fits to obtain the pillar thickness. Next, the thickness is derived using

$$
\delta_{p i}=\frac{F_{A F M} L^{3}}{3 E I_{p i}}, \text { with } I_{p i}=\frac{\pi}{4} r_{p i}^{4},
$$

and $L$ and $I_{p i}$ being the probe distance and the moment of inertia, respectively. Since the microstructure of the pillar is unknown, a (bulk) Young's modulus $E$ of $117 \mathrm{GPa}$ is assumed. Finally, to cross-check this approach and to assess whether the 

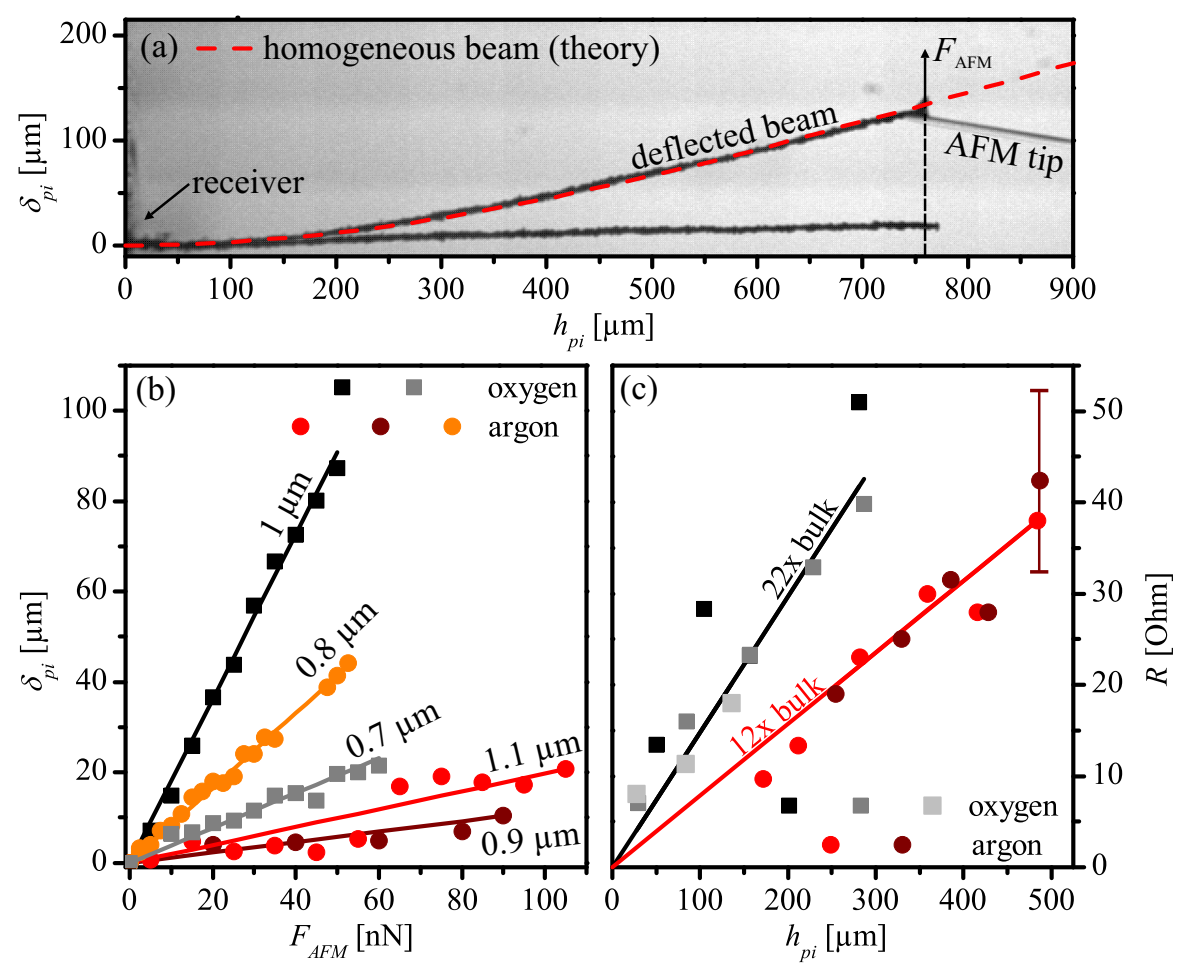

Figure 8.4: (a) Optical microscope image of a pillar with a height of $h_{p i} \approx 800 \mu \mathrm{m}$, which is deflected by the tip of an AFM. The arrow at the tip of the AFM lever indicates the force (here $F_{A F M}=90 \mathrm{nN}$ ). The dashed line represents the modeled deflection of a theoretical homogeneous beam with a diameter of $2.2 \mu \mathrm{m}$. (b) Measuremed deflection of the pillar tip as a function of the AFM force applied. The shown linear dependency of the deflection on the applied force corresponds to elastic deformation of a beam with a homogeneous thickness. The deflection $\delta_{p i}$ is measured at a different pillar height for each pillar, as indicated next to each line. (c) Electrical resistance of pillars processed in air and argon as a function of the pillar height. The slope of the fitted lines indicates the resistance per unit length, which is 22 and 12 times higher than for bulk copper for the air- and argon-processed pillars, respectively. The error resulting from the $\approx 20 \%$ uncertainty in pillar thickness is indicated, and scales proportional to $R_{p i}$.

pillars are homogeneous in thickness, the shape of the deflected pillar is compared to the theoretical solution for a bent beam of homogeneous thickness according to equation (8.1). The dashed line in figure 8.4 (a) indicates a bended beam of homogeneous thickness, which coincides with the deflected pillar. This confirms that the pillar is effectively homogeneous in thickness, despite its surface roughness and internal voids. The deformation of the pillar is elastic, illustrating that the pillar can be significantly deformed prior to plastic deformation.

The electrical resistance of the pillars is plotted in figure 8.4 (c). The resistance 
was measured for different pillar heights at different stages in the deposition process as illustrated in figure 8.4 (c). The slope indicates the pillar resistance per unit length, which is normalized by the pillar area to provide the effective bulk resistance $\rho_{E}$. The electrical properties of the pillars are measured by connecting electrodes to the donor- and receiver substrates, and gently pressing the donor layer onto the tip of the pillar, such that electrical contact is guaranteed. In this approach, the circuit is closed and the resistance can be measured. The resistance consists of the contact resistance $R_{c}$ and the resistance of the pillar itself. By measuring this resistance during the deposition process - i.e. for pillars of increasing length, increasing values of the resistance are obtained. The slope of the linear fit of the $R_{p i}(h)$ curve, which was corrected for $R_{c}$, provides the pillar resistance per unit length.

For pillars processed in oxygen atmosphere at room conditions, $\rho_{E}=35.3$. $10^{-8} \Omega \mathrm{m}$, which exceeds the resistance of bulk copper, which is $\rho_{E}=1.6 \cdot 10^{-8} \Omega \mathrm{m}$, by a factor of 22 . As oxidation of the metal droplets or the pillar tip during deposition was expected to cause this relatively high resistance, a next set of pillars was processed in an argon environment. As can be concluded from figure 8.4 (c), this reduces the bulk resistivity to $\rho_{E}=18.7 \cdot 10^{-8} \Omega \mathrm{m}$, thus almost halves the resistance as compared to the air-processed pillars. The value of bulk copper is not obtained, presumably due to transfer of copper oxide from the donor film, or invisible nanometer-scale inhomogeneities within the pillar. Those inhomogeneities may origin from for example: gas bubbles that are entrapped during droplet deposition [124]. Still, as both these values are several orders of magnitude below other metals used for printing of conductive wires, e.g. Gallium-Indium, for which $\rho_{E}=3 \cdot 10^{-3} \Omega \mathrm{m}$ [125], the pillars printed here are for example suitable for out-of-plane electrode manufacturing.

\subsubsection{Demonstrators and printing applications}

To illustrate example applications of LIFT, the pillar deposition concept is extended in the following. In figure 8.5 (a), a $860 \mu \mathrm{m}$ long pillar is deposited, by moving the receiver down during droplet deposition, while maintaining a distance between the donor and the pillar tip in the range of $20 \mu \mathrm{m}$ to $50 \mu \mathrm{m}$. Even higher pillars can easily be created, until at some height the position of the tip is no longer stable. That is, small vibrations or light air flows result in a "waving" motion of the tip. Therefore a wider distribution in the droplet impact location with respect to the tip is observed, resulting in a tip with a larger diameter. The longest homogeneous pillar produced is $2100 \mu \mathrm{m}$ high and less than $5 \mu \mathrm{m}$ in radius (see figure $8.3(\mathrm{c})$ ), providing an aspect ratio of at least 1:200. This pillar was deposited in gold. Next, the pillars can be deposited as out-of-plane interconnects between different layers of stacked electronics. A top view and cross section of such a filled TSV are shown in figure 8.5 (f) and 8.5 (g), respectively. The top view shows the virtually clean deposition process. That is, only a few droplets landed next to the TSV as indicated by the arrows. The cross-section of the TSV shown in figure $8.5(\mathrm{~g})$, verifies that the pillar extends to the $100 \mu \mathrm{m}$ deep bottom. So far, only a single a filled TSV was prepared as for visualization, as their preparation without damaging the pillar proved to be highly challenging - e.g. the 


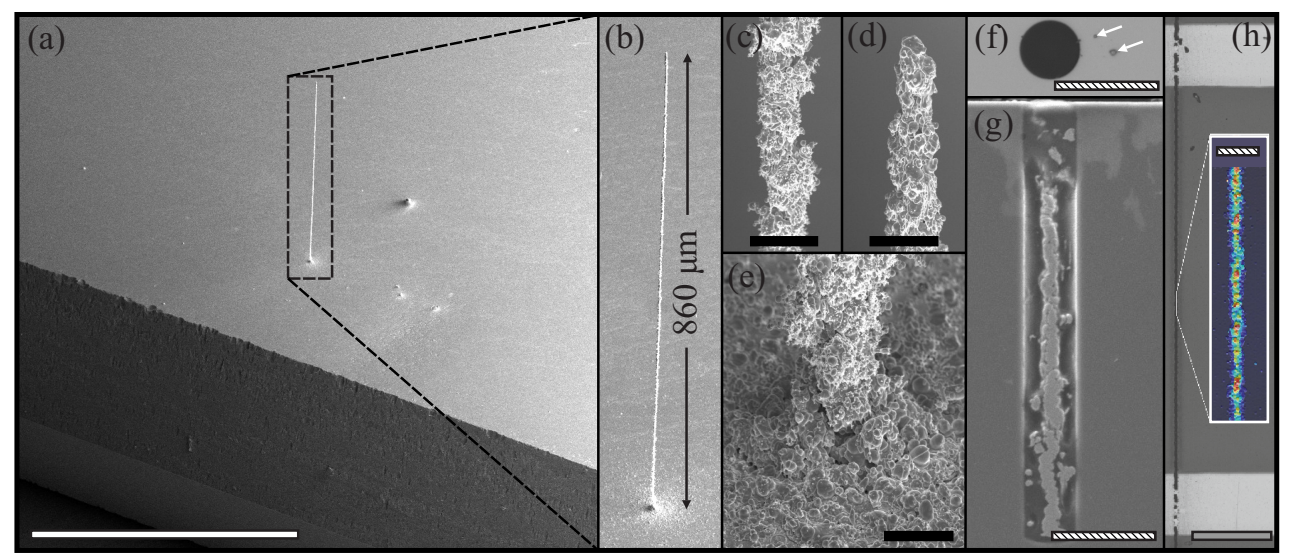

ᄃ $1 \mathrm{~mm} 200 \mu \mathrm{m} 20 \mu \mathrm{m}=5 \mu \mathrm{m}$

Figure 8.5: High-aspect ratio examples of copper LIFT. (a) $860 \mu \mathrm{m}$ long pillar close to the clearly visible edge of a microscope slide. (b) Close-up of the pillar, showing that the pillar has a virtually homogeneous thickness: the center (c) and top (d) of the pillar are approximately $4 \mu \mathrm{m}$ in diameter whereas the bottom diameter (e) is about $6 \mu \mathrm{m}$, due to droplets landing next to the pillar. Figures (f) and (g) show a top view and a cross section of a filled TSV, respectively. The dark area next to the pillar (also visible from top) is a polymer for fixating the pillar, to avoid any damage due to polishing. The arrows in (f) indicate droplets that landed next to the TSV. The cross-section in image (g) shows that the pillar extends to the bottom of the hole. Finally, by moving the receiver substrate while depositing droplets, conductive lines can be fabricated as shown in figure (h). The inset shows a close-up of the line, in which the colors represent a height map.

defect close to the top in figure 8.5 (g) might be caused by accidentally cutting the intact pillar. Finally, high aspect ratios can also be achieved in the horizontal plane, e.g. printing of a conductive copper line, shown in figure $8.5(\mathrm{~h})$. This conductive line was achieved by moving the receiver substrate, while repeatedly ejecting droplets. By controlling the receiver position also in the horizontal plane, truly $3 \mathrm{D}$ structures become in reach.

\subsection{Discussion}

The disk-shaped droplets that constitute the pillars seem to be a novel solidification regime in LIFT. For fluence levels just above the ejection threshold a sphere is deposited, as shown in figure 8.6 (a) and reported previously [10, 16-18, 42, 49, 50, $126,127]$. For intermediate fluence levels, the droplets solidify as a disk, shown in figures 8.6 (b) and 8.6 (c). Toroids [18] and fragmentation of droplets [49, 128] have also been observed, whereas splashing is only scarcely observed. For even higher fluence levels, a transition occurs to spray ejection, in which many droplets are ejected and deposited. Still, these droplets individually solidify as a sphere, disk, or toroid as shown in figures 8.6 (d) and e.g. by Banks et al. [18]. Since the droplet 


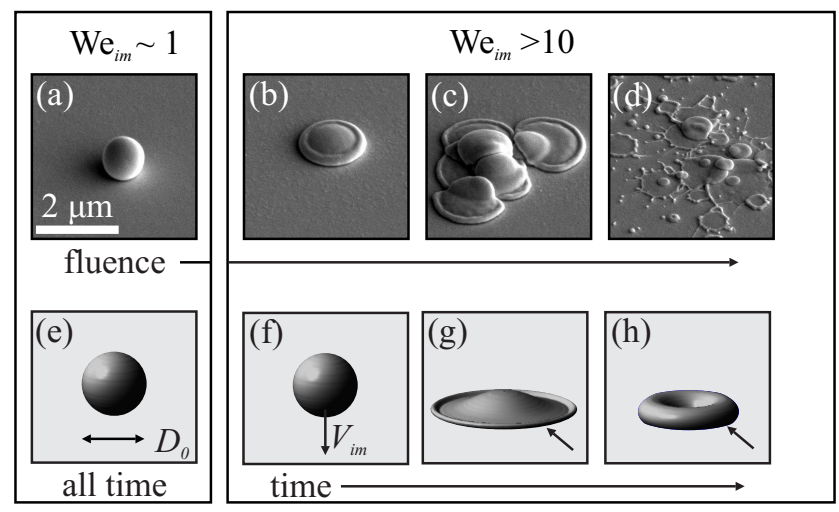

Figure 8.6: Droplet shapes as a function of fluence, impact Weber number $\left(\mathrm{We}_{i m}\right)$, and time. SEM-Images (a) to (d) show solidified droplets deposited on a copper-coated surface, for laser fluences of $380,440,630$, and $820 \mathrm{~mJ} / \mathrm{cm}^{2}$, respectively. Image (a) shows a droplet deposited due to fluence levels just above the ejection fluence threshold. For these droplets the Weber number is about 1, resulting in spherical solidification. For increasing fluence, droplets are deposited as a disk, which is used to construct the pillars. Finally, a transition to spray ejection occurs resulting in the ejection of many droplets, as shown in (d). In this regime, the solidification patterns include toroids. The scale bar of $2 \mu \mathrm{m}$ holds for all images. Figures (e) to (h) show numerical simulations of impact dynamics onto non-wetting surfaces. For numerical details see Visser et al. [124]. Figure (e) illustrates low Weber number impact - here, $\mathrm{We}_{i m}=1$. In this low-velocity regime, the impact is dominated by surface tension and the droplet maintains a spheroid shape. (f) to (h) illustrates the spreading dynamics of an impacting droplet for the high Weber number case - here, $\mathrm{We}_{i m}=150$. Figure (f) shows the droplet approaching the surface with velocity $V_{i m}$. (g) The maximal spreading diameter is reached on the inertial timescale $\approx 2 V_{i m} / D_{0} .(\mathrm{h})$ Subsequently, the droplet retracts (snapshot shown for $t=5 V_{i m} / D_{0}$ ) and the rim becomes dominant.

shape will affect the properties of any LIFT-printed structure, but has received scarce attention in literature, this shape will now be discussed for various cases.

To assess why spherical deposition is usually observed for fluence levels just above the ejection threshold, first the ejection velocity at the ejection fluence threshold is calculated. Next, it is subsequently shown that this velocity is insufficient to force the droplet into a disk shape. Ejection occurs if the inertia of the ejected material exceeds the surface tension of the film, which is quantified by a threshold ejection Weber number [123]

$$
\mathrm{We}_{e j}=\frac{\rho d V_{e j}^{2}}{\sigma} \approx 1
$$

with donor film thickness $d$, density $\rho$, surface tension $\sigma$, and ejection velocity $V_{e j} \approx \sqrt{\sigma / \rho d}$. Next, to determine the droplet shape during impact, an impact Weber number $\mathrm{We}_{i m}=\rho D_{0} V_{i m}^{2} / \sigma$ is defined, describing the ratio between inertia and surface energy of the impacting droplet. The diameter $D_{0}$ of the droplet is generally of the same order as the film thickness $D_{0}<10 \mathrm{~d}$. The effect of air drag is 
neglected and it is assumed that $V_{i m}=V_{e j}$, providing a maximum impact Weber number of $\mathrm{We}_{i m} \approx 10$. For these low Weber numbers, droplets hardly spread during impact [129] as shown in figure 8.6 (e), and thus can only solidify in a spherical shape. Since fluences just above the ejection threshold also correspond to the smallest droplets $[10,16-18,42,49,50,126,127]$, increasing the printing resolution using smaller droplets and control of the droplet shape are conflicting requirements.

For higher laser fluences, the impact velocity increases (i.e. $\mathrm{We}_{i m} \gtrsim 10$ ), resulting in inertial impact illustrated in figures 8.6 (f) to 8.6 (h). An additional requirement for inertial impact is a high ratio of kinetic impact energy to viscous dissipation, which is represented by the Reynolds number $\operatorname{Re}=\rho D_{0} V_{i m} / \eta$, with viscosity $\eta$. In LIFT, this condition is fulfilled since usually $\operatorname{Re} \gtrsim 100$. Initially, the droplet spreads out into a disk, until a certain maximal spreading diameter is reached as shown in figure $8.6(\mathrm{~g})$. Meanwhile, the edge of the droplet is contracting by surface tension, which results in the formation of an initially small "rim" shown in figure $8.6(\mathrm{~g})$. This rim keeps growing until the droplet reaches an essentially toroidal shape as shown in figure 8.6 (h). Eventually, the droplet contracts back into a spheroid that can even bounce away from the surface [124]. Those spheres, disks, and toroids observed in LIFT (see figures 8.6 (a) to 8.6 (d)) thus correspond to droplet shapes for non-solidifying impact.

The particular solidified droplet shape will depend on the solidification timescale with respect to the spreading and retraction timescales of the droplet. However, the solidification timescale cannot yet reliably be calculated. Therefore an empirical approach is employed to optimize the droplet shape. Initially, the ejected droplets were deposited onto a glass receiver, but then the droplets bounce away. Since this bouncing effect was previously attributed to evaporation of volatile elements in the glass [130], the glass receiver was coated with a $100 \mathrm{~nm}$ thick copper film (see section 4.3), resulting in disk-shaped solidification. This approach is sufficient for pillar deposition, but it is to be expected that further optimization of the film thickness and material allows to optimize the solidification timescale and thereby allow for a deposition of the droplet in any of the shapes discussed.

As discussed, a trade-off exists between control of the droplet shape, its impact location, and contamination. For fluences around the ejection threshold, clean deposition can be achieved but deposition will be spherical [10, 16-18, 42, 49, 50, $126,127]$ due to the low Weber number. Moreover, droplet stacking in this regime could not be achieved, since the impact location was insufficiently controlled for donor-receiver distances of $10 \mu \mathrm{m}$ to $100 \mu \mathrm{m}$. For fluence levels that significantly exceed the ejection threshold, the droplet shape can be controlled as discussed in the previous paragraph, but here a single laser pulse generally results in the ejection of multiple droplets. Remarkably, when significantly exceeding the ejection threshold fluence, these droplets primarily land on top of each other as shown in figure 8.6 (c). The presented pillars have been deposited in this regime, since this behavior is consistent and the location of impact is robust with respect to the ejection location. 


\subsection{Summary}

In summary, the deposition of copper and gold droplets using LIFT was successfully controlled, in order to construct pillars, lines, and fill through-silicon vias. The pillars, with diameters below $5 \mu \mathrm{m}$ and lengths over $2000 \mu \mathrm{m}$, show a low porosity, are electrically conductive, and are mechanically homogeneous. The pillars consist of disk-shaped solidified droplets, which was found to be beneficial for 3D printing since the drop-to-drop contact area and adhesion are maximized. It was shown that disks are only obtained for laser fluence levels that substantially exceed the ejection fluence threshold, and explained why spherical deposition was observed in previous work. Remarkably, droplet shape control and maximizing the printing resolution seem to be mutually exclusive requirements. By controlling both the position and angle of the receiver substrate, micro-manufacturing of truly $3 \mathrm{D}$ structures will be achievable. 


\section{Chapter 9}

\section{Solid phase LIFT of variable shapes}

In this chapter* the LIFT of thin donor layers in solid phase is addressed. An improved beam shaping setup is employed to achieve full flexible beam shaping capability. Thereby, the transfer of the donor in flexible shapes is achieved and demonstrated for various shapes.

\subsection{Introduction}

In the previous chapters 5 to 8 , the ejection process in liquid phase has been discussed in detail and employed to achieve high aspect ratio printing. However, certain applications may benefit from the deposition of unmelted donor, i.e. the transfer of the donor layer in solid phase. In this context, the LIFT process can be categorized in broadly two classes, analog to the physical condition of the ejected donor - i.e. either transfer of the donor in liquid phase (see chapters 5, 6, and 7) or solid phase, as illustrated in figure 9.1. For liquid phase transfer, the irradiated part of the donor layer is melted by the absorbed laser energy to form droplets with dimensions smaller than the diameter of the focal spot $[16,18]$. In the case of solid phase LIFT, the ablation of a thin part of the donor layer near the carrier-donor interface [131] results in the delamination of the donor layer from the carrier substrate. Alternatively to ablation, thermally induced stress waves inside the donor layer [54] can cause the delamination of the latter. The part of the donor layer that is transferred in solid phase is usually referred to as a "pellet". Currently, solid phase transfer faces several problems. Large shearing forces, occurring at the pellet perimeter during transfer, limit the similarity between the desired pellet shape and the deposited pellet shape. Furthermore, the deposited pellet may be surrounded by

*Parts of this chapter have been published in Applied Physics A, published online, 2015, R. Pohl, M. Jansink, G.R.B.E. Römer, A.J. Huis in 't Veld. Solid-phase laser-induced forward transfer of variable shapes using a liquid crystal spatial light modulator. 

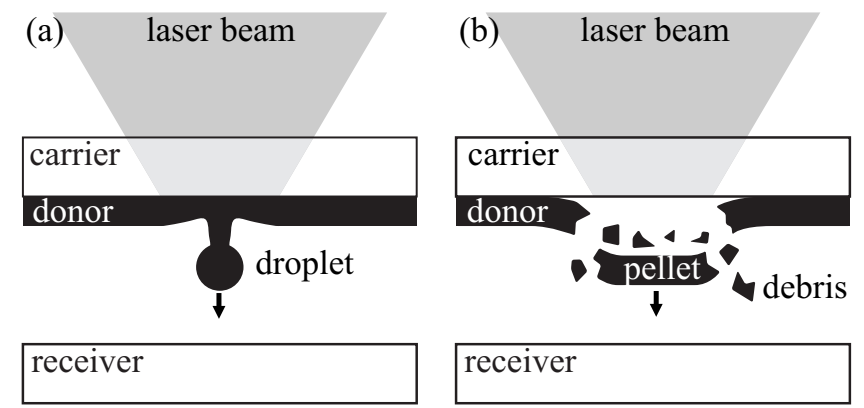

Figure 9.1: Schematic representation of LIFT (adapted from [131]), where the absorbed laser energy (a) melts the donor layer resulting in droplet formation [16, 18] or (b) transfers a solid phase "pellet" due to either ablating a thin part of the donor layer [131] or thermally inducing stress waves inside the donor layer [54].

debris particles formed by undesired transferred donor material.

This chapter aims to achieve fast, solid phase pellet transfer of thin donor layers of variable shapes. Such a transfer technique can be used in applications that require the donor material to remain intact during transfer. Clearly, the LIFT process as indicated in figure 9.1 (a) is not suitable for those applications as the donor material is transferred in liquid phase. However, LIFT of pellets as shown figure 9.1 (b) has drawbacks that complicate the pellet transfer in variable shapes, namely:

- The ejection and transfer of a pellet is typically so "violent" that often the pellet shatters or even disintegrates.

- Large shearing forces, occurring at the pellet perimeter during transfer, limit the similarity between the desired pellet shape and the deposited pellet shape.

- The pellet shape is directly related to the spatial intensity profile of the laser beam, referred to in the following as the beam shape. Variable pellet shapes therefore require spatially shaped beams.

- Thin donor layers are easily melted. To achieve solid phase pellets these pellets should be transferred as a result of the thermally induced stress waves, as studied by Shugaev et al. [54].

Previous work has attempted to solve these issues. Tolbert et al. [55] introduced a Dynamic Release Layer (DRL) located between the carrier and the donor material, thereby protecting the latter during transfer, see chapter 2 for more details. Laser Decal Transfer (LDT), investigated by Piqué et al.[56], uses a viscous suspension of metallic nano-particles as the donor layer. This so called nano-ink or nano-paste is irradiated by a flat-topped laser beam, using aperture masks to achieve limited flexibility in pellet shape. Auyeung et al.[57, 132] improved LDT by using a Digital Micro-mirror Device (DMD) for spatial beam shaping. DMDs are able to modulate 
the intensity of the incident laser beam in a binary fashion (on or off) on a per pixel basis and are used to vary the pellet shape on demand. Ballistic Laser-Assisted Solid Transfer (BLAST), as demonstrated by Banks et al.[58], uses the laser out of its focal plane (defocused) to achieve beam shapes with a higher intensity perimeter that directly weakens the shearing areas of the donor layer. A focused ion beam (FIB) technique is employed by Kaur et al.[59] to pre-machine the donor layer in the desired pellet shape. The LIFT process is then used to transfer only the pre-machined part of the donor layer. Recently, Rapp et al.[133] demonstrated the advantages of mask-based Smart Beam Shaping (SBS) on the transfer of PEDOT:PSS pixles, using optimized beam shapes with overexposed perimeters. Though, this beam shaping method in principle allows for a variety of beam shapes, it is limited in flexibility due to the time consuming mask preparation and the lack of real-time modulation of the desired beam shape.

Neither of these approaches solve all the problems mentioned above. While DRL-LIFT protects the pellet from damage during the LIFT process, it does not address the shearing forces that limit the fidelity of the pellet shape. In contrast, LDT has been used to achieve great flexibility in pellet shape, but this approach is not suitable for applications that require truly solid donor material. BLAST allows solid donor material, but it is limited to mostly circular pellet shapes. The pre-machining step for FIB is time-consuming and therefore costly, although it has the advantage that it provides flexibility in pellet shape. The mask-based SBS allows for flexible beam shapes, but is lacking real-time flexibility due to the working principle of the mask projection.

This chapter introduces a novel approach using a liquid crystal spatial light modulator (SLM) in the LIFT process to achieve solid phase pellet transfer of variable shapes. In contrast to DMDs, that only perform binary intensity modulation, an SLM offers full grayscale intensity modulation when used in a cross-polarization setup [134, 135]. The details of the setup are discussed in section 9.2 . Section 9.3 provides a proof-of-concept in the form of successfully transferred solid phase pellets of varying shapes from $100 \mathrm{~nm}$ thick gold donor layers. Inspired by BLAST [58] and the LIFT of pre-machined donor layers [59], single- and double-pulse experiments with modulated laser beams according to the desired pellet shapes are presented.

\subsection{Methods}

The following two subsections provide a detailed explanation of the SLM beam shaping setup and the preparation of the donor and receiver samples that are used in this work. To this end, the principle of operation of the beam shaping setup is described by three steps: irradiation of the SLM with the laser beam, modulation of the intensity profile through polarization based phase modulation "on a per pixel basis", and imaging of that profile onto the sample through an infinity optical microscope. 


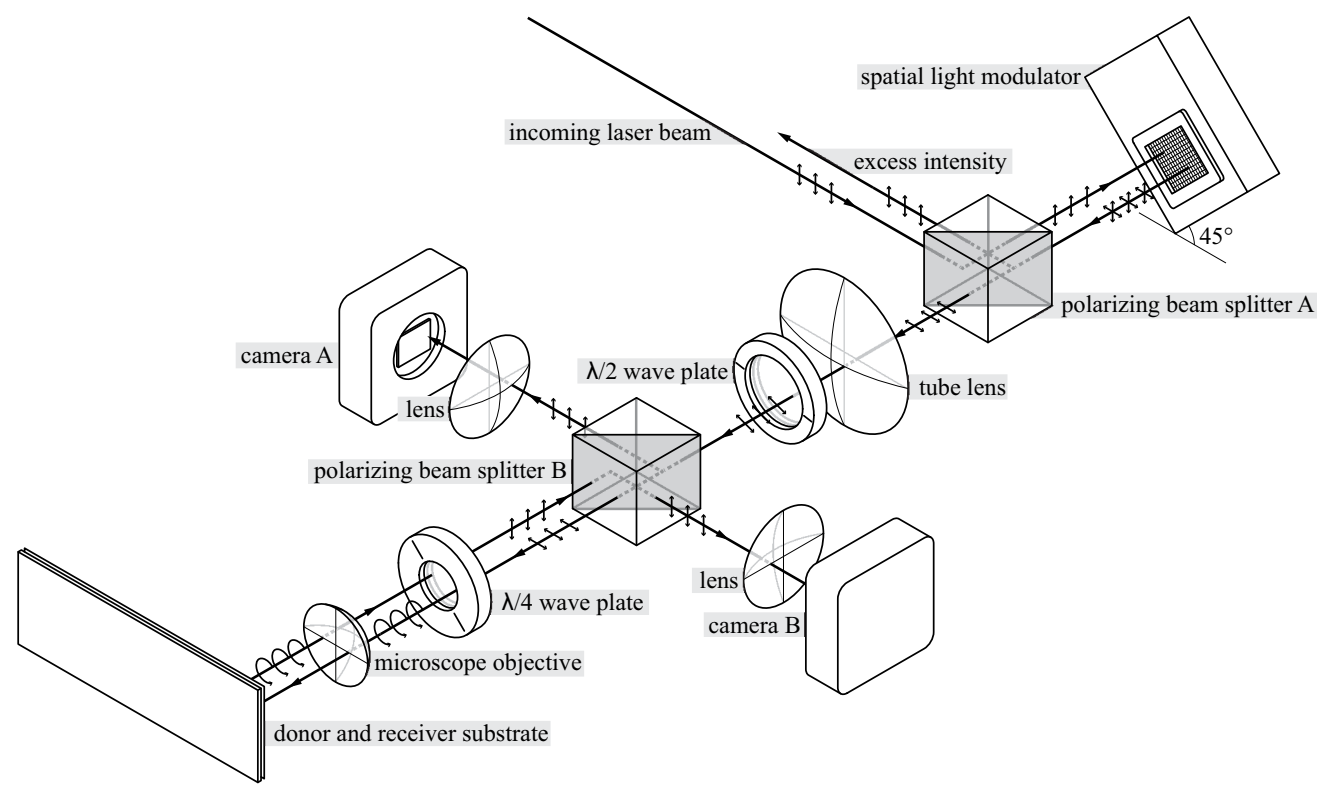

Figure 9.2: Schematic sketch of the experimental setup for solid phase LIFT. Only to guide the readers eye, different "arms" of the laser beam are offset-ed with respect to the optical axis to allow the indication of the different polarization states by double headed arrows.

\subsubsection{SLM beam shaping}

Figure 9.2 shows the cross-polarization setup employed in this work. A 6.7 picosecond laser is used at a pulse repetition rate of $40 \mathrm{~Hz}$, see section 4.2 .4 for details. The setup uses the unfocused laser beam, which diameter is determined to be approximately $6 \mathrm{~mm}$ maintaining a linearly polarization in the vertical direction. The Polarizing beam splitter A (ThorlaBs CM1-PBS251) directs the beam towards the SLM (Hamamatsu X10468-02 [136]).

The SLM consists of $800 \times 600$ pixels on an active area of 16 by $12 \mathrm{~mm}$, resulting in a pixel size of $20 \times 20 \mu \mathrm{m}$. Each pixel can be controlled individually on a grayscale of 8 bits. The birefringence effect of the crystals induces a wavelength dependent phase retardance in the horizontal component of the incident beam with respect to the SLM, which is mounted at an angle of $45^{\circ}$. Custom software was developed to control the grayscale pattern of the SLM, which allows for full control of the status of polarization on the per-pixel basis. Light reflected by the SLM passes through the polarizing beam splitter A a second time. In this case, the splitter transmits only the horizontally polarized component of the laser beam. This ultimately allows for the full intensity modulation of the laser beam.

The surface of the SLM, i.e. the shaped laser beam, is imaged onto the carrierdonor interface using a demagnifying microscope setup. The microscope consists of an infinity-corrected tube lens (Thorlabs ITL200, $f=200 \mathrm{~mm}$ ) in combina- 

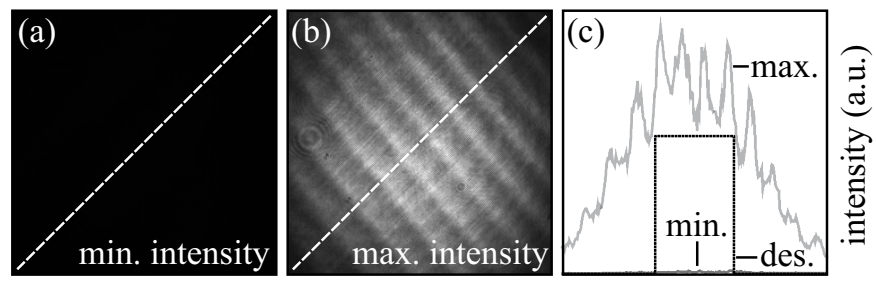

Figure 9.3: Laser beam profiles captured by camera A after respectively (a) minimizing and (b) maximizing the intensity using the SLM. The grayscale level in these images is a measure for the intensity. The intensity on the dashed lines in profiles (a) and (b) is plotted together with a desired, in this case, flat-topped beam shape in graph (c). The latter is fitted within the maximum intensity; depending on the beam shape, energy efficiencies of up to $50 \%$ can be achieved. In both, profile (b) and graph (c) interference fringes caused by the polarizing beam splitters can be observed.

tion with a $100 \times$ infinity-corrected long working distance microscope objective (Mitutoyo Plan Apo \#46-147). The latter is mounted on a translation stage (NEWPORT 562-XYZ) for positioning of the focus location relative to the carrierdonor interface. The spatial resolution of this set-up is determined by the resolving power of the microscope objective, and is specified to be $0.4 \mu \mathrm{m}$. However, due to aberrations caused by the carrier and due to the optical components placed into the beam path, this diffraction limited resolution will not be achieved, but is provided as the best case scenario.

For inline profiling of the beam shape, a half-wave plate (THORLABS WPH10M514 ) on a rotatable mount is placed between the tube lens and the microscope objective. Polarizing beam splitter B (ThorLABS CM1-PBS251) deflects an adjustable part of the intensity of the beam through a lens (THORLABs LA1509-A, $f=100 \mathrm{~mm}$ ) on camera A (ThORLABS DCC1545M). This camera captures a high-dynamic range image to measure a single beam profile on the SLM. These images are first used to calibrate the SLM in order to correct for any deformations of its surface. The resulting minimum and maximum intensity profiles are shown in Figure 9.3, where the maximum profile contains a factor of 60 more energy than the minimum profile. At the wavelength of $515 \mathrm{~nm}$, the phase difference of one $\pi$ between minimum and maximum intensity corresponds to a gray level change of 47 on the SLM, limiting the intensity resolution to some extent. The interference pattern observable in the maximum intensity profile, as shown in figures 9.3 (b) and 9.3 (c), is likely attributed to "ghost reflections" at the interfaces (beam path SLM to Camera A) of the polarizing beam splitters and mainly attributed to the performance of the broad band anti-reflection coatings that are applied on each interface. However, both issues have little effect on the experimental results.

The desired beam shape is fitted within the maximum intensity profile as shown in figure 9.3 (c) and the required intensity modulation is calculated by the software in an iterative approach. That is, using a first-order approximation of the relation between gray level and phase retardance, the initial pattern of the SLM 

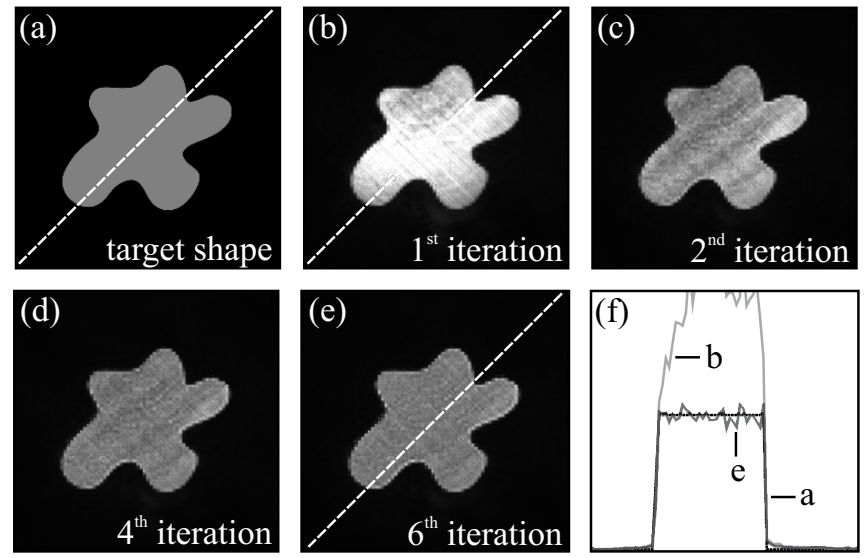

Figure 9.4: Laser beam profiles captured by camera A after several iterations of the software. The grayscale level in these images is a measure for the intensity of the beam shape. The arbitrary chosen desired beam shape is shown in profile (a), profiles (b) to (e) show the beam shape after respectively the initial, $2^{\text {nd }}, 4^{\text {th }}$ and $6^{\text {th }}$ iteration. The intensity on the indicated lines in profiles (a), (b) and (e) is plotted in graph (f), showing that the beam shape "converges" to the desired beam shape after six iterations of the controller. The RMS error between the desired beam shape and the measured beam shape after the $6^{\text {th }}$ iteration is $8 \%$.

is calculated. A proportional feedback controller, inspired by Liang et al.[137] is applied, which iteratively improves the required phase pattern by comparing the desired beam shape to the beam shape measured by camera A. Figure 9.4 shows this measured beam shape after several iterations of the feedback loop for an arbitrary desired beam shape. Depending on the desired beam shape, the reshaped laser beam contains up to $50 \%$ of the energy of the laser beam incident on the SLM.

Camera B (Thorlabs DCC1545M) captures light that is reflected from the carrier-donor interface back through the microscope objective. To this end, a quarter-wave plate (THORLABS WPDM05M-1064H-532Q) is placed between polarizing beam splitter B and the objective. A lens (ThorLABs LA1509-A $f=100$ $\mathrm{mm}$ ) is used to image the carrier-donor interface onto the camera. Being able to view the surface of the donor inline and in real-time brings two major advantages. First, it allows the focusing of the beam profile on the carrier-donor interface to be adjusted optically. Secondly, it easily allows inspection of the donor after each pulse. For the latter, an additional (cold-) light source was used to illuminate the donor surface.

\subsubsection{Sample preparation and experimental methods}

The donor carrier slide is a $60 \times 24 \times 0.13 \mathrm{~mm}$ hydrolytic class 1 glass slide (MENzEL GLÄSER BB024060A1). A $100 \mathrm{~nm}$ donor layer of gold is deposited on this slide 

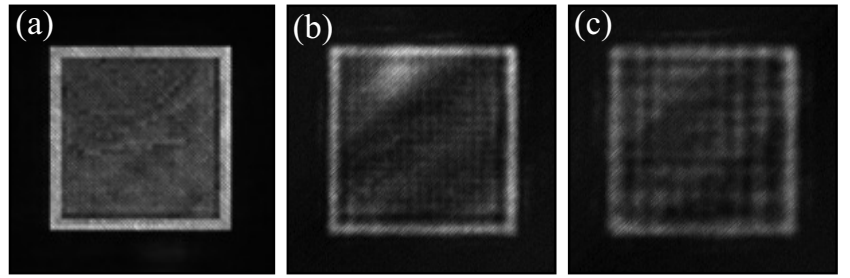

Figure 9.5: Laser beam profiles. The grayscale level in these images is a measure for the beam intensity. Profile (a) is captured by camera A directly after it is shaped by the SLM and shows clear distinction between a desired higher fluence perimeter and the lower fluence interior of the beam shape. Both profiles (b) and (c) are captured by camera B and show the beam shape after it has been reflected by the donor layer. For profile (b) a $0.13 \mathrm{~mm}$ glass slide is used, while for profile (c) a $1.0 \mathrm{~mm}$ glass slide is used. The difference in beam shape between profiles (b) and (c) is caused by the different glass thicknesses.

using magnetron sputtering running at a sputter rate of $23 \mathrm{~nm} / \mathrm{min}$, see section 4.3 for details. These thin glass slides are chosen to minimize the influence of spherical aberrations leading to a deterioration of the laser beam shapes, as illustrated figure 9.5 .

The receiver is based on a standard $76 \times 26 \times 1 \mathrm{~mm}$ microscope slide on which a $400 \mathrm{~nm}$ gold coating is deposited using the same sputter technique as to create the donor layer. The same material is used for both the donor and receiver layers to optimize the adhesion of the transferred solid phase pellets on the receiver. The donor and receiver are stacked with the coated sides parallel and facing each other. The separation between the donor and the receiver is set to $50 \mu \mathrm{m}$, using thin metal foils placed between both substrates. This stack is placed on a XYZ translation stage (ThORLABS PT3) that is partially motorized using two actuators (THORLABs Z825B). The translation is controlled and synchronized with the release of single laser pulses ensuring proper donor refreshment during processing.

\subsection{Results}

The experimental setup is used for two types of experiments, using either a singlepulse process or double-pulse process to achieve solid phase pellet transfer. For the first set of experiment, a single pulse ejection is investigated. Here, the laser beam is shaped according to a desired pellet shape. The fluence level of the perimeter of the beam is optimized such that it completely melts the irradiated part of the donor layer. In contrast, the fluence level at the interior of the beam is optimized to trigger the ejection process, exploiting thermally induced stresses in the donor layer.

The second set of experiment demonstrates the ejection of solid pellets, using two laser pulses. The first pulse is shaped such that it removes an area of the donor layer around the desired pellet shape. The second laser pulse uses a flat-top beam shape to transfer this remaining pellet. The results of these experiments are discussed in the following subsections 9.3.1 and 9.3.2. 

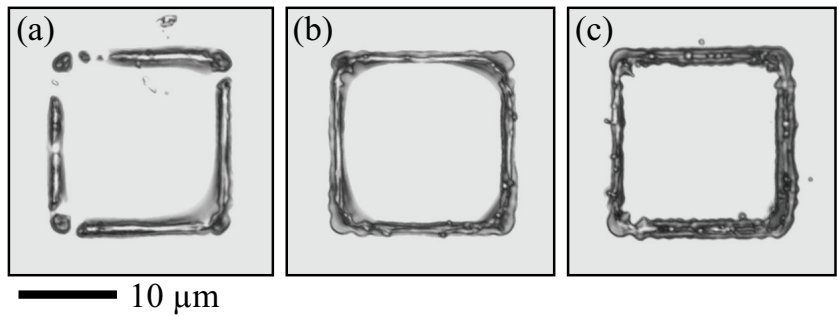

Figure 9.6: Donor substrate imaged using confocal microscopy after single-pulse experiments to determine the required border fluence for a square cutout. Sample (a) is processed with a too low fluence level of $210 \mathrm{~mJ} / \mathrm{cm}^{2}$, resulting in an incomplete cutout, while for (c) the fluence level of $270 \mathrm{~mJ} / \mathrm{cm}^{2}$ is too high resulting in a broad molten border around the desired pellet shape. The fluence level of $240 \mathrm{~J} / \mathrm{cm}^{2}$ used for (b) is found to be optimal for pellet transferring of $100 \mathrm{~nm}$ gold donors.

\subsubsection{Single-pulse process}

In order to limit the shearing forces in the donor layer during ejection process, the beam shape is optimized for the desired pellet shape. Therefore, the beam shape resembles the desired pellet shape surrounded by a perimeter at a fluence level that suffices to completely melt the donor layer, similar to [58]. In contrast, the fluence level in the interior of the beam is optimized to prevent melting, while the ejection process is initiated by a thermally induced stress wave that leads to the ejection of a pellet. It is assumed that the heat flow in the donor is one-dimensional. That is, heat propagates only in the propagation direction of the laser beam. This allows the two fluence levels to be determined sequentially as neither region of the beam shape should affect the other.

The optimal fluence levels are determined using a simple square as the desired pellet shape. The corresponding beam shape including the overexposed perimeter is shown in figure 9.5 (a). To first determine the optimal fluence of the perimeter of the beam shape, the fluence in the interior is chosen zero. Several representative results showing the donor layer are shown in figure 9.6. At low fluence levels, such as $210 \mathrm{~mJ} / \mathrm{cm}^{2}$ in figure 9.6 (a), the perimeter is not completely cut out. The observed asymmetry is attributed to the deterioration of the beam shape inside the carrier slide, as demonstrated in figure 9.5. The use of fluence levels higher than $240 \mathrm{~mJ} / \mathrm{cm}^{2}$ results in a broad cutout which indicates that the one-dimensional heat flow assumption may not be completely valid. The optimal fluence is found at $240 \mathrm{~mJ} / \mathrm{cm}^{2}$, as this fluence level results in a full cutout of the perimeter with minimum melting. With the perimeter fluence level fixed to $240 \mathrm{~mJ} / \mathrm{cm}^{2}$, the interior fluence level is varied as a percentage of the perimeter fluence to determine the optimal fluence level to achieve solid phase pellet transfer. The effect of the interior fluence level is determined by analyzing the deposited pellets on the receiver, see Figure 9.7. From these results the optimal fluence level of the interior is found to be approximately $20 \%$ of the border fluence or $48 \mathrm{~mJ} / \mathrm{cm}^{2}$. It is clear that the results are quite sensitive to variations in this fluence. That is, fluence levels smaller than $48 \mathrm{~mJ} / \mathrm{cm}^{2}$ cause too little delamination 

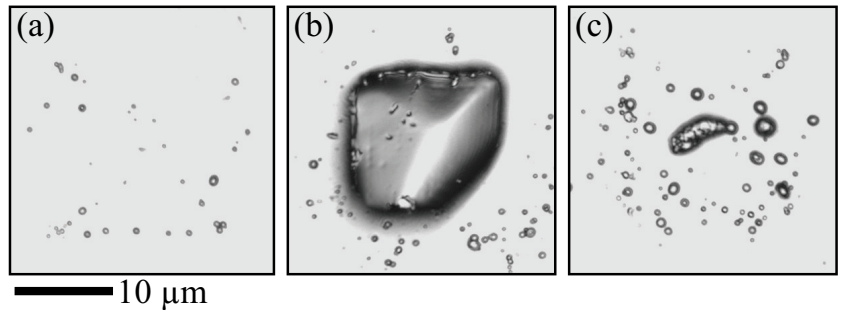

Figure 9.7: Receiver substrate imaged using confocal microscopy after single-pulse experiments to determine the required center fluence for transfer of a solid square. Sample (a) is processed with a too low fluence level of $26 \mathrm{~mJ} / \mathrm{cm}^{2}$, causing the donor layer not to transfer, while for (c) the fluence level of $64 \mathrm{~mJ} / \mathrm{cm}^{2}$ is too high resulting in the complete melting of the pellet. The fluence level of $48 \mathrm{~mJ} / \mathrm{cm}^{2}$ used for (b) is found to be optimal for $100 \mathrm{~nm}$ gold donors.

of the donor and/or too little thrust to transfer a pellet from the donor to the receiver layer (figure 9.7 (a)), while fluence levels higher than $48 \mathrm{~mJ} / \mathrm{cm}^{2}$ result in the complete melting of the pellet, see figure 9.7 (c).

This is consistent with an estimation of the minimum fluence level $F$, that is required to achieve full melting of the donor layer. This minimum fluence level can be calculated as

$$
F=\frac{d\left(C_{p} \Delta T+\rho L_{m}\right)}{1-R},
$$

with the donor layer thickness $d=100 \mathrm{~nm}$, the specific heat capacity $C_{p}=2.5$. $10^{6} \mathrm{~J} /\left(\mathrm{m}^{3} \mathrm{~K}\right)$, density $\rho=19300 \mathrm{~kg} / \mathrm{m}^{3}$, latent heat of fusion $L_{m}=0.6 \cdot 10^{5} \mathrm{~J} / \mathrm{kg}$, and reflectivity $R=0.6$ for the given wavelength of $515 \mathrm{~nm}$ wavelength [138, 139]. It was found that the perimeter fluence level of $F=240 \mathrm{~mJ} / \mathrm{cm}^{2}$ is sufficient to melt the donor layer, which is in accordance with the experimental results. Furthermore, it can be shown that experimentally determined optimal fluence level of $\left(F=48 \mathrm{~mJ} / \mathrm{cm}^{2}\right)$ for the interior leads to a temperature raise of $\Delta T \approx 800 \mathrm{~K}$. Therefore, no melting occurs and it is concluded that the transfer of the pellet is a result of the thermally induced stress waves as studied by Shugaev et al.[54].

To demonstrate the repeatability of this method, a large amount of transfers is made using the optimized fluence levels. Both square and circular desired pellet shapes are chosen and the receiver is imaged, see figure 9.8. It should be noted that these results show that the experiments consistently lead to transferred solid phase pellets shaped and sized accordingly to the desired pellet shape and corresponding beam shape. Especially the shape of the circular pellets shows a high similarity to the corresponding desired shape. However, the square pellets have rounded corners, which is a result from the fact that relatively more energy is transferred to the donor layer as two parts of the perimeter meet at a corner. This causes a larger heat up of the donor layer in a corner and therefore slightly more donor material is molten.

Furthermore, it can be observed that all pellets are not flat, but show some consistent deformation. In general, the center of the pellet is raised higher with respect to its perimeter, which may indicate that the center of the pellet was 


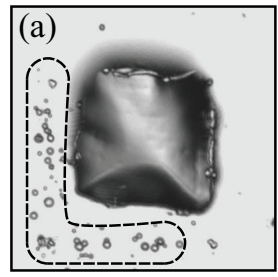

(c)

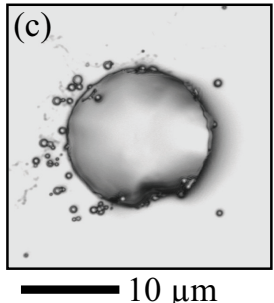

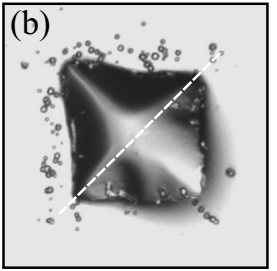

(d)

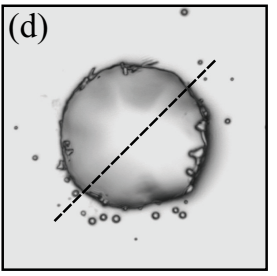

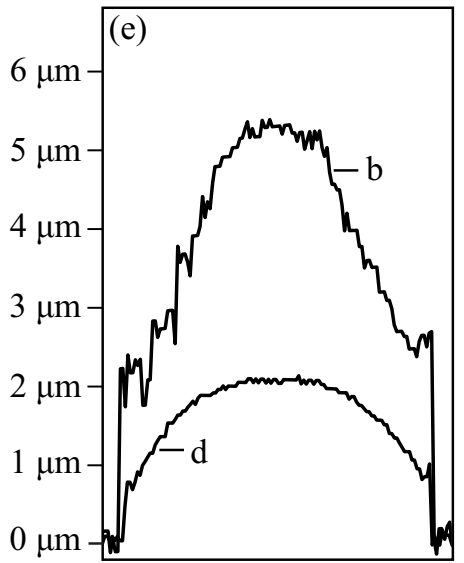

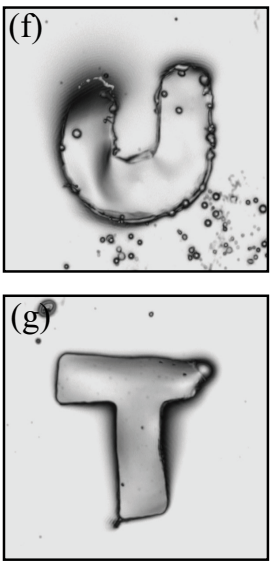

Figure 9.8: Receiver substrate imaged using confocal microscopy after single-pulse experiments at optimal fluence levels of $240 \mathrm{~mJ} / \mathrm{cm}^{2}$ for the perimeter and $48 \mathrm{~mJ} / \mathrm{cm}^{2}$ for the interior of the beam shape. Samples (a) and (b) correspond to a square desired pellet shape of $19.2 \mu \mathrm{m}$ sides, samples (c) and (d) correspond to a circular desired pellet shape 9.6 $\mu \mathrm{m}$ in diameter. It should be noted that all pellets show a similar deformation in which the center of each pellet is raised on average approximately $3 \mu \mathrm{m}$ above its perimeter. This is illustrated in graph (e) for the dashed lines shown on sample (b) and (d). The debris around the transferred shapes is caused by the melting of the perimeter of the pellet shape. On sample (a), this debris is enclosed within a dashed curve. Samples (f) and (g) demonstrate the flexibility in desired pellet shapes achievable, here the characters "U" and "T".

the last area to detach from the carrier slide. A possible cause for the latter is some unexpected heat diffusion parallel to the surface of the donor layer. This would cause the areas close to perimeter of the beam shape to heat up faster and delaminate earlier from the carrier and the observed deformation is caused by the bending of the very thin pellets. The deformation may also be caused by air drag during the transfer of the pellet from the carrier to the receiver, or a combination of both effects.

The observed bending is expected to have a significant impact on the overall adhesion between the deposited pellets and the receiving substrate. However, in the framework of this thesis the adhesion was only exploratory studied and reduced to two scenarios, i.e. the deposition on an uncoated glass receiver and the deposition on a gold coated sample as described in section 9.2.2. Experiments on depositing solid gold pellets on a glass surface were unsuccessful. That is, the transferred pellets did not stick to the receiver surface but were found at random positions on the receiver, after process. In contrast, the deposition on a gold coated surface was proven to be successful as the deposited pellets stick to the surface - i.e. robust enough to handle them without special care in a laboratory environment.

Finally, debris is found around the deposited pellet. This is to be expected, as completely melting the perimeter of the shape of the pellet will generate molten 

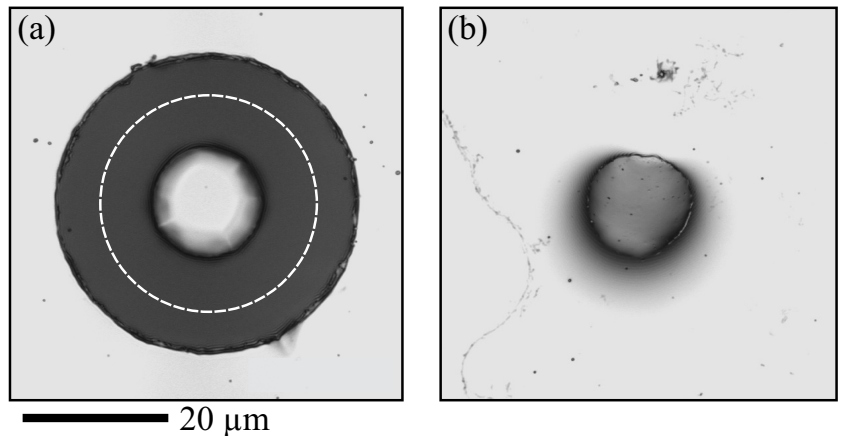

Figure 9.9: Donor layer (a) and receiver substrate (b) imaged using confocal microscopy after double-pulse experiments. First, an area around the pellet is removed from the donor layer after which a second laser pulse transfers that pellet intact. The perimeter of the laser beam shape used for transfer is indicated with the dashed line in (a).

donor material that will end up on the receiver layer. Even though, it can be considered detrimental to the final result.

To further demonstrate the possibilities of the current setup, more elaborate desired pellet shapes were chosen. More specifically, pellets shaped like the characters "U" and "T" (an acronym for the University of Twente) were transferred with the same optimized fluence levels as detailed above. The receiver layer is imaged, see figure 9.8 (f) and $9.8(\mathrm{~g})$. The deposited pellets show some deformation, but form a clearly legible "U" and "T", providing a proof of the flexibility in pellet shapes that this setup provides. Considering the " $\mathrm{T}$ " to be composed of two perpendicular "bars", each bar is approximately $5 \mu \mathrm{m}$ width and $16 \mu \mathrm{m}$ long. The left part of the "T" in figure $9.8(\mathrm{~g})$ shows radii of approximately $1 \mu \mathrm{m}$, which represent the resolution of the current setup. However, it should be mentioned that this feature size may not be the highest resolution of the current setup.

\subsubsection{Double-pulse process}

To achieve solid phase pellet transfer without debris, the cutout of the perimeter of the desired pellet shape and the actual transfer of the pellet are performed sequentially using two separate laser pulses. That is, the laser beam for the first pulse shows an annular shape such that it removes an area of the donor layer around the desired pellet shape, see figure 9.9 (a). For this pre-machining step, the receiver is removed from the experimental setup to prevent any molten donor material from contaminating it. The perimeter that is cut out is relatively broad compared to the pellet size, with an outer radius of $21.6 \mu \mathrm{m}$ and an inner radius of $7.2 \mu \mathrm{m}$ respectively, see figure 9.9 (a). This allows for a high tolerance in alignment of the laser beam with the remaining donor material in the center.

Next, the beam shape that is used to transfer the pellet is circular, flat-topped and has a radius which is twice as large as the radius of the pellet, at a fluence level 
of $51 \mathrm{~mJ} / \mathrm{cm}^{2}$. An example of such a transferred pellet is shown in figure 9.9 (b), and there is no observable debris around the deposited pellet.

This process requires the use of multiple pulses to achieve solid phase LIFT, much as like it is proposed in previous work $[58,59]$. The novel technique described in this work, however, is an improvement on either previous approaches. It uses a precisely controlled beam shape to provide greater flexibility in and control over the resulting pellet shape. Furthermore, the required number of pulses to transfer the pellet is known a priori (namely exactly two) and only the required fluence level has to be determined. A clear advantage is the implementation using a single setup, without requiring other equipment such as the focused ion beam used by Kaur et al.[59]. This approach gives a real-time flexibility in pellet shape, however pre-machining can of course still be performed by some other donor preparation techniques.

\subsection{Summary}

This chapter presented a novel approach using a liquid crystal spatial light modulator (SLM) in the LIFT process to achieve solid phase pellet transfer of variable shapes. To this end, intensity profiles consisting of a low fluence interior surrounded by a high fluence perimeter were investigated. The optimal laser fluence levels, to achieve solid transfer of an $100 \mathrm{~nm}$ thick gold layer, were experimentally determined and found to be $F=0.24 \mathrm{~J} / \mathrm{cm}^{2}$ and $F=48 \mathrm{~mJ} / \mathrm{cm}^{2}$ for the interior and the perimeter, respectively. Aiming at the transfer of square and circular shaped pellets, the laser beam was shaped accordingly and many solid phase pellets were successfully deposited using the determined optimal fluence levels. Although undesired debris was frequently observed, the shape of these deposited pellets was found to closely match the desired pellet shapes. More complex shapes like the characters "U" and "T" were successfully deposited and provided as a proof for the flexibility in shape and the spatial resolution that can be obtained with this beam shaping method.

In order to transfer and deposit pellets without debris, a double-pulse process was employed. This approach was proven successful, as debris was completely absent at the resulting deposited pellets. Hence, it is concluded that this approach of laser-induced forward transfer shows great potential for debris free solid transfer of micrometer sized 2D shapes of thin films. 


\section{Chapter 10}

\section{Conclusions and recommendations}

In this chapter, the research objectives as formulated in the first chapter will be addressed and complemented by recommendations regarding further improvements of the LIFT process.

\subsection{Conclusions}

- Objective 1: Investigate the driving mechanisms that initiate the ejection process during LIFT.

In order to investigate the driving mechanisms during LIFT, high resolution highspeed imaging experiments were performed to visualize fluence dependent ejection dynamics and hence to identify and characterize fluence regimes of ejections during picosecond LIFT. Varying the laser fluence $F$ revealed different ejection regimes, which were illustrated for a $200 \mathrm{~nm}$ copper film. For $320 \mathrm{~mJ} / \mathrm{cm}^{2}<F<600$ $\mathrm{mJ} / \mathrm{cm}^{2}$, ejection of a hemispherical feature of the film was observed, referred to as "cap"-ejection. For $600 \mathrm{~mJ} / \mathrm{cm}^{2}<F<740 \mathrm{~mJ} / \mathrm{cm}^{2}$ "jet-ejection" occurs, since here a narrow jet leading the cap apex was observed. For $F>740 \mathrm{~mJ} / \mathrm{cm}^{2}$ a cloud of particles was observed, called "spray ejection". To interpret these ejection regimes, the physical conditions in the donor layer were assessed. Therefore, the response of the material to the absorbed laser pulse was computed. To that end, a numerical two-temperature model, as explained in detail in chapter 3. From these computations, two driving mechanisms, namely laser-induced stress relaxation and the vaporization of the donor layer were discussed. These mechanisms were confirmed by energy balances, which were employed to provide a simple model predicting the velocities of the ejections. Good agreement of these computations with velocity measurements for copper and gold films was obtained, using a material-dependent fitting constant. The observed minimal ejection velocity of $\approx 20 \mathrm{~m} / \mathrm{s}$ was found to correspond to a Weber number of We $\approx 1$. From this it 
was concluded that at lower ejection velocities (at which We $<1$ ), surface tension retracts the liquid film and no donor material is ejected.

- Objective 2: Investigate the ejection dynamics during LIFT that lead to the generation of undesired deposits on a receiving substrate.

The origin of undesired contamination during picosecond LIFT was addressed in chapter 6. To this end, SEM images of the ejection craters after LIFT, and time-resolved image sequences at laser fluence levels corresponding to the cap, jet and spray ejection regimes were discussed.

For cap ejection, the generation of additional undesired droplets was found to be correlated to the separation of the ejected cap from the donor layer. That is, the subsequent formation and break-up of liquid threads along the rupture line of the ejected cap were proposed to cause the formation of additional droplets, next to the ejected cap. Moreover, the driving mechanisms of the ejection process were discussed in more detail. The analysis of the ejection crater suggests a one-dimensional ejection process, which is characterized by the generation of a transversal stress wave. Accordingly, the shape of the ejected caps was proposed to resemble the geometrical shape of the Gaussian profile of the focused laser pulse. To this end, a qualitative description of the cap ejection process was provided.

Next, the jet ejection regime was assessed following the proposed one-dimensional approach. From that, it was shown that the absorbed laser pulse ultimately causes a velocity gradient across spatial profile of the ejected jet. This profile was attributed to the geometrical shape of the Gaussian beam profile and the onset of vaporization in the center of the ejection crater. The proposed ejection mechanism provided a reasonable explanation for the dynamics that ultimately lead to the full fragmentation of the ejected jets. This fragmentation leads to the formation of many relatively small droplets and was shown to be the dominant mechanism in order to explain the massive contamination in this fluence regime.

Finally, the ejection dynamics of the spray ejection regime were discussed. It was found that the captured ejection dynamics resemble the characteristics of explosive-boiling, as discussed in prior work by Zhigilei et. al. [116]. To this end, the donor ejection due to homogenous melting was considered and the spray ejection during picosecond LIFT was fully attributed to the dominance of explosive boiling.

- Objective 3: Study the effect of the laser pulse duration on the ejection process during LIFT.

Chapter 7 addressed the effect of the pulse duration on the observed ejection regimes during LIFT. Therefore, an experimental study using nanosecond LIFT was presented and discussed. Here, two ejection regimes have been observed. For laser fluence values just above the transfer threshold of $280 \mathrm{~mJ} / \mathrm{cm}^{2}$, the formation of a liquid gold jet and the subsequent formation and ejection of single and multiple droplets were shown. Increasing laser fluence, increases the number of ejected droplets. For laser fluence levels above $800 \mathrm{~mJ} / \mathrm{cm}^{2}$, the formation and rupture of a blistering bubble was observed. This regime was found to be less suitable for controlled (i.e. 
clean) deposition, since multiple droplets are always ejected at angles that cannot be controlled as yet. A description of the low fluence jet ejection regime was provided and compared to the observed cap ejection during picosecond LIFT. The influence of the pulse duration was discussed with respect to the donor layer thickness and the established temperature gradient that is generated across the thickness of the donor layer. From that, the different ejection mechanism were found to be directly related to the generated stress distribution in the donor layer, and hence directly related to the generated temperature gradient in latter. It was shown that depending on the layer thickness and the pulse duration either a strong transversal stress wave across the donor layer, or a stress gradient perpendicular to the optical axis are generated. It was concluded, that depending on those gradients either cap like (picosecond) or jet-like (nanosecond) ejections are triggered. Moreover, it was hypothesized that each regime can be triggered, regardless the pulse duration, as long as the layer donor thickness is chosen accordingly with respect to the generated temperature gradient.

- Objective 4: Explore the droplet impact during LIFT and identify the crucial parameters with respect to $3 \mathrm{D}$ printing.

The impact dynamics during LIFT and especially the properties of 3D printed structures were addressed in chapter 8. Therefore, the deposition of liquid copper and gold droplets using LIFT was successfully controlled, to construct pillars, lines, and to fill through-silicon vias. The pillars, with diameters below $5 \mu \mathrm{m}$ and lengths up to $2000 \mu \mathrm{m}$, exhibit a low porosity, were found to be electrically conductive, and mechanically homogeneous. Those pillars were printed with disk-shaped solidified droplets, which was found to be beneficial for $3 \mathrm{D}$ printing since the drop-to-drop contact area and adhesion are maximized. It was shown that disks are only obtained for laser fluence levels that substantially exceed the ejection fluence threshold, and it was hypothesized why spherical deposition was observed in previous work. To this end, the physical conditions during the solidification of the impacting droplet were accessed. Remarkably, drop shape control and maximizing the printing resolution were found to be mutually exclusive requirements.

- Objective 5: Explore the capabilities of LIFT for solid transfer of thin metal films of arbitrary shapes.

The solid phase transfer, using LIFT was addressed in chapter 9. It was demonstrated that a spatial light modulator can be effectively used to apply intensity modulation using a cross-polarization setup. The optimal intensity profile consists of a high fluence perimeter around an interior characterized by a lower fluence level to avoid melting of the thin donor layer. The ejection was triggered by the thermally induced stress wave, that delaminates the donor from the carrier. Using both square and circular pellet shapes, the laser beam was shaped accordingly and many solid phase pellets were successfully deposited using the determined optimal fluence levels. The shape of these deposited pellets were found to match the desired pellet shapes. Pellets shaped like the characters "U" and "T", with a resolution of $\approx 1 \mu \mathrm{m}$, were successfully deposited and provide a proof of the flexibility in pellet shape that can be obtained. 
Fully clean and debris free depositions were demonstrated using a double-pulse ejection process, which effectively decoupled the cut-out of the perimeter from the ejection process. From these results, it was concluded that this novel approach to laser-induced forward transfer shows great potential for solid transfer of micrometer sized 2D shapes without debris.

\subsection{Recommendations}

This thesis provided a broad discussion on the topic of LIFT of metals, including the driving mechanisms and first demonstrators of 3D printing of high melting point metals. However, extended research is required to achieve full 3D printing capability. Although the demonstrated pillars exhibit an impressive aspect ratio, those structures resemble the simplest structure of 3D printing. Fully 3D requires the stitching of the droplets also in the horizontal plane, which is an issue that has not been addressed in this work, but is mandatory to achieve full 3D printing.

Moreover, the droplet impact and the resulting shape of the solidified droplet is currently poorly controlled. The presented pillars were successfully printed exploiting the cap ejection regime, as it allows for torus-like solidified droplets. However, the shape is determined by two parameters, namely impact velocity and the thermal boundary conditions of the impacting droplet. The thermal conditions are usually fixed by the donor itself, as the material is deposited subsequently on itself to build-up a 3D structure. Therefore, a control on the ejection velocity is needed in order to control the shape of the solidified droplets. To this end, the velocity may be exploited to optimize the microstructure and hence the mechanical and electrical properties of the printed structure.

The LIFT of certain (pure) metals like copper, still suffer from the stochastic generation of undesired droplets (satellites). Although, the demonstrated jet-like ejections, as usually achieved for thin layers, result in almost clean depositions, improved control on the number of ejected droplet and the ejection angle of the latter may be of relevance to achieve fully clean, fast and flexible printing of metal droplets. 


\section{Bibliography}

[1] Ian Gibson, David W. Rosen, and Brent Stucker. Additive Manufacturing Technologies, volume 76. Springer US, Boston, MA, 2010.

[2] B.-J. de Gans, P.C. Duineveld, and U.S. Schubert. Inkjet printing of polymers: State of the art and future developments. Advanced Materials, 16(3):203-213, February 2004.

[3] Savas Tasoglu and Utkan Demirci. Bioprinting for stem cell research. Trends in Biotechnology, 31(1):10-9, January 2013.

[4] K. Yamaguchi. Generation of 3-dimensional microstructure by metal jet. Microsystem Technologies, 9(3):215-219, January 2003.

[5] Collin Ladd, Ju-Hee So, John Muth, and Michael D Dickey. 3D printing of free standing liquid metal microstructures. Advanced Materials (Deerfield Beach, Fla.), 25(36):5081-5, September 2013.

[6] Jolke Perelaer, Patrick J. Smith, Dario Mager, Daniel Soltman, Steven K. Volkman, Vivek Subramanian, Jan G. Korvink, and Ulrich S. Schubert. Printed electronics: the challenges involved in printing devices, interconnects, and contacts based on inorganic materials. Journal of Materials Chemistry, 20(39):8446, 2010.

[7] Bong Kyun Park, Dongjo Kim, Sunho Jeong, Jooho Moon, and Jang Sub Kim. Direct writing of copper conductive patterns by ink-jet printing. Thin Solid Films, 515(19):7706-7711, July 2007.

[8] Seung Hwan Ko, Jaewon Chung, Nico Hotz, Koo Hyun Nam, and Costas P Grigoropoulos. Metal nanoparticle direct inkjet printing for low-temperature 3D micro metal structure fabrication. Journal of Micromechanics and Microengineering, 20(12):125010, December 2010. 
[9] Jacob J. Adams, Eric B. Duoss, Thomas F. Malkowski, Michael J. Motala, Bok Yeop Ahn, Ralph G. Nuzzo, Jennifer T. Bernhard, and Jennifer A. Lewis. Conformal printing of electrically small antennas on three-dimensional surfaces. Advanced Materials (Deerfield Beach, Fla.), 23(11):1335-40, March 2011.

[10] Gerrit Oosterhuis, An Prenen, and A. J. Huis in 't Veld. Laser induced forward transfer of interconnects for 3D integration. ECS transactions, 41(43):81-90, 2012.

[11] J. Bohandy, B. F. Kim, and F. J. Adrian. Metal deposition from a supported metal film using an excimer laser. Journal of Applied Physics, 60:1538 - 1539, 1986 .

[12] P. Mogyorósi, T. Szörényi, K. Bali, Zs. Tóth, and I. Hevesi. Pulsed laser ablative deposition of thin metal films. Applied Surface Science, 36:157-163, 1989.

[13] Z. Tóth, T. Szörényi, and A. L. Tóth. Ar ${ }^{+}$laser-induced forward transfer (lift): a novel method for micrometer-size surface patterning. Applied Surface Science, 69:317-320, 1993.

[14] M. Wagner V. Schultze. Blow-off aluminum films. Applied Physics A, 53:241$248,1991$.

[15] Hilmar Esrom, Jun-Ying Zhang, Ulrich Kogelschatz, and Anthony J. Pedraza. New approach of a laser-induced forward transfer for deposition of patterned thin metal films. Applied Surface Science, 86:202 - 207, 1995.

[16] Vicentiu Grosu David A. Willis. Microdroplet deposition by laser-induced forward transfer. Applied Physics Letters, 86:244103, 2005.

[17] I. Zergioti, S. Mailis, N. A. Vainos, P. Papakonstantinou, C. Kalpouzos, C. P. Grigoropoulos, and C. Fotakis. Microdeposition of metal and oxide structures using ultrashort laser pulses. Applied Physics A, 66:579-582, 1998.

[18] David P. Banks, Christos Grivas, John D. Mills, Robert W. Eason, and Ionna Zergioti. Nanodroplets deposited in microarrays by femtosecond ti:sapphire laser-induced forward transfer. Applied Physics Letters, 89:193107, 2006.

[19] Chris Germain, Luc Charron, Lothar Lilge, and Ying Y. Tsui. Electrodes for microfluidic devices produced by laser induced forward transfer. Applied Surface Science, 253(19):8328-8333, July 2007.

[20] F. Roozeboom, M. Smets, B. Kniknie, M. Hoppenbrouwers, G. Dingemans, W. Keuning, W.M.M. Kessels, R. Pohl, and A.J. Huis in 't Veld. Alternative technology concepts for low-cost and high-speed 2D and 3D interconnect manufacturing. In 46th IMAPS International Symposium on Microelectronics, 2013. 
[21] D. Young, R. C. Y. Auyeung, A. Piqué, D. B. Chrisey, and Dana D. Dlott. Plume and jetting regimes in a laser based forward transfer process as observed by time-resolved optical microscopy. Applied Surface Science, 197-198:181-187, 2002 .

[22] M. Duocastella, J.M. Fernández-Pradas, P. Serra, and J. L. Morenza. Jet formation in the laser forward transfer of liquids. Applied Physics A, 93:453-456, 2008 .

[23] M. Duocastella, J. M. Fernández-Pradas, J. L. Morenza, and P. Serra. Timeresolved imaging of the laser forward transfer of liquids. Journal of Applied Physics, 106(8):084907, 2009.

[24] Matthew S. Brown, C. Frederik Brasz, Yiannis Ventikos, and Craig B. Arnold. Impulsively actuated jets from thin liquid for high-resolution printing applications. Journal of Fluid Mechanics, 709:341 - 370, 2012.

[25] Christos Boutopoulos, Ioannis Kalpyris, Efthymis Serpetzoglou, and Ioanna Zergioti. Laser-induced forward transfer of silver nanoparticle ink: time-resolved imaging of the jetting dynamics and correlation with the printing quality. Microfluidics and Nanofluidics, 16(3):493-500, August 2013.

[26] Romain Fardel, Matthias Nagel, Frank Nuesch Thomas Lippertesch, Thomas Lippert, and Alexander Wokaun. Laser-induced forward transfer of organic led building blocks studied by time-resolved shadowgraphy. Physical Chemistry $C$, 114:5617-5636, 2010.

[27] Matthias Domke, Stephan Rapp, Michael Schmidt, and Heinz P. Huber. Ultrafast movies of thin-film laser ablation. Applied Physics A, 109(2):409-420, 2012.

[28] D. Bartl, M. Ametowobla, F. Schmid, A. Letsch, M. Hafner, S. Nolte, and A. Tünnermann. Probing timescales during back side ablation of molybdenum thin films with optical and electrical measurement techniques. Optics Express, 21(14):16431-43, July 2013.

[29] M. Feinaeugle, A.P. Alloncle, Ph. Delaporte, C.L. Sones, and R.W. Eason. Time-resolved shadowgraph imaging of femtosecond laser-induced forward transfer of solid materials. Applied Surface Science, 258:8475 - 8483, 2012.

[30] S. A. Mathews, R. C. Y. Auyeung, H. Kim, N. a. Charipar, and A. Piqué. Highspeed video study of laser-induced forward transfer of silver nano-suspensions. Journal of Applied Physics, 114(6):064910, 2013.

[31] Y. Nakata and T. Okada. Time-resolved microscopic imaging of the laserinduced forward transfer process. Applied Physics A, 69:275 - 278, 1999.

[32] T. Sano, H. Yamada, T. Nakayama, and I. Miyamoto. Experimental investigation of laser induced forward transfer process of metal thin films. Applied Surface Science, 186:221 - 226, 2002. 
[33] Anthony B. Bullock and Paul R. Bolton. Laser-induced back ablation of aluminum thin films using picosecond laser pulses. Journal of Applied Physics, 85(1):460-465, 1999.

[34] I. Zergioti, D. G. Papazoglou, A. Karaiskou, C. Fotakis, E. Gamaly, and A. Rode. A comparative schlieren imaging study between ns and sub-ps laser forward transfer of cr. Applied S, 208 - 209:177 - 180, 2003.

[35] Zsolt Tóth, Bela Hopp, Tamas Szörényi, Zsolt Bor, Elena A. Shakhno, and Vadim P. Veiko. Pulsed laser ablation mechanisms of thin metal films. In Proceedings of SPIE, volume 3822, pages 18-26, September 1999.

[36] V. P. Veiko, E. A. Shakhno, V. N. Smirnov, A. M. Miaskovski, and G. D. Nikishin. Laserinduced film deposition by lift physical mechanisms and applications. Laser and Particle Beams, 24:203 - 209, 2006.

[37] Claudia Unger, Martin Grüne, Lothar Koch, Jürgen Koch, and Boris N. Chichkov. Time-resolved imaging of hydrogel transport by laser-induced forward transfer (lift). In Proceedings pf LPM2010 - the 11th International Symposium on Laser Precision Microfabrication, 2010.

[38] D. S. Ivanov and L. V. Zhigilei. Combined atomistic-continuum modeling of short-pulse laser melting and disintegration of metal films. Physical Review B, 68:064114, 2003.

[39] C. Mézel, L. Hallo, a. Souquet, J. Breil, D. Hébert, and F. Guillemot. Selfconsistent modeling of jet formation process in the nanosecond laser pulse regime. Physics of Plasmas, 16(12):123112, 2009.

[40] D. S. Ivanov, A. I. Kuznetsov, V. P. Lipp, B. Rethfeld, B. N. Chichkov, M. E. Garcia, and W. Schulz. Short laser pulse nanostructuring of metals: direct comparison of molecular dynamics modeling and experiment. Applied Physics A, 111(3):675-687, March 2013.

[41] Tobias C. Röder and Jürgen R. Köhler. Physical model for the laser induced forward transfer process. Applied Physics Letters, 100:071603, 2012.

[42] Arseniy I. Kuznetsov, Claudia Unger, Jürgen Koch, and Boris N. Chichkov. Laser-induced jet formation and droplet ejection from thin metal films. Applied Physics A, 106:479 - 487, 2012.

[43] A. Piqué, D. B. Chrisey, R. C. Y. Auyeung, J. Fitz-Gerald, H. D. Wu, R. A. McGill, S. Lakeou, P. K. Wu, V. Nguyen, and M. Duignan. A novel laser transfer process for direct writing of electronic and sensor materials. Applied Physics A, 69:279 - 284, 1999.

[44] B. Hopp, T. Smausz, Zs. Antal, N. Kresz, Zs. Bor, and D. Chrisey. Absorbing film assisted laser induced forward transfer of fungi (trichoderma conidia). Applied Physics, 96:3478, 2004. 
[45] Graciela B. Blanchet, Yueh-Lin Loo, J. A. Rogers, F. Gao, and C. R. Fincher. Large area, high resolution, dry printing of conducting polymers for organic electronics. Applied Physics Letters, 82:463, 2003.

[46] Z. Kántor and T. Szörényi. Dynamics of long-pulse laser transfer of micrometersized metal patterns as followed by time-resolved measurements of reflectivity and transmittance. Applied Physics, 78:2775, 1995.

[47] Hiroshi Fukumura, Yoshiharu Kohji, Ken-ichi Nagasawa, and Hiroshi Masuhara. Laser implantation of pyrene molecules into poly(methyl methacrylate) films. American Chemical Society, 116:10304 - 10305, 1994.

[48] Merijn P. Giesbers, M. B. Hoppenbrouwers, E. C. P. Smits, and R. Mandamparambil. Process optimization of lift through visualization: towards high resolution metal circuit printing. Proc. SPIE, Laser Sources and Applications II, 9135:91350Z, May 2014.

[49] Li Yang, Ching yue Wang, Xiao chang Ni, Zhi jun Wang, Wei Jia, and Lu Chai. Microdroplet deposition of copper film by femtosecond laser-induced forward transfer. Applied Physics Letters, 89:161110, 2006.

[50] Christina M. Othon, Arnaldo Laracuente, H.D. Ladouceur, and Bradley R. Ringeisen. Sub-micron parallel laser direct-write. Applied Surface Science, 255(5):3407-3413, December 2008.

[51] H. Yamada, T. Sano, T. Nakayama, and I. Miyamoto. Optimization of laserinduced forward transfer process of metal thin films. Applied Surface Science, 197 - 198:411 - 415, 2002.

[52] T. V. Kononenko, P. Alloncle, V. I. Konov, and M. Sentis. Shadowgraphic imaging of laser transfer driven by metal film blistering. Applied Physics A, 102(1):49-54, October 2010.

[53] I. Zergioti, A. Karaiskou, D. G. Papazoglou, C. Fotakis, M. Kapsetaki, and D. Kafetzopoulos. Time resolved schlieren study of sub-pecosecond and nanosecond laser transfer of biomaterials. Applied Surface Science, 247:584 $-589,2005$.

[54] Maxim V. Shugaev and Nadezhda M. Bulgakova. Thermodynamic and stress analysis of laser-induced forward transfer of metals. Applied Physics A, 101:103 - 109, 2010.

[55] William A Tolbert, I-Y Sandy Lee, MM Doxtader, EW Ellis, and DD Dlott. High-speed color imaging by laser ablation transfer with a dynamic release layer: fundamental mechanisms. The Journal of Imaging Science and Technology, 37(4):411-421, 1993.

[56] Alberto Piqué, Raymond C.Y. Auyeung, Heungsoo Kim, Kristin M. Metkus, and Scott A. Mathews. Digital microfabrication by laser decal transfer. Journal of Laser Micro / Nanoengineering, 3(3):163-168, 2008. 
[57] R.C.Y. Auyeung, H. Kim, N.A. Charipar, A.J. Birnbaum, S.A. Mathews, and A. Piqué. Laser forward transfer based on a spatial light modulator. Applied Physics A, 102(1):21 - 26, 2011.

[58] David P. Banks, Christos Grivas, Ioanna Zergioti, and Robert W. Eason. Ballistic laser-assisted solid transfer (blast) from a thin film precursor. Optics Express, 16:3249 - 3254, 2008.

[59] K. S. Kaur, M. Feinaeugle, D. P. Banks, J. Y. Ou, F. Di Pietrantonio, E. Verona, C. L. Sones, and R. W. Eason. Laser-induced forward transfer of pre-machined donor films. Optical Sociaty of America, 2011.

[60] F. J. Adrian, J. Bohandy, B. F. Kim, and A. N. Jette. A study of the mechanism of metal deposition by the laser-induced forward transfer process. Journal of Vacuum Science and Technology B, 5:1490 - 1494, 1987.

[61] Vahit Sametoglu, Vincent T. K. Sauer, and Ying Y. Tsui. Production of 70-nm cr dots by laser-induced forward transfer. Opt. Express, 21(15):18525-18531, Jul 2013.

[62] E. Fogarassy, C. Fuchs, F. Kerherve, G. Hauchecorne, and J. Perrière. Laserinduced forward transfer of high- $\mathrm{Tc} \mathrm{YBaCuO}$ and $\mathrm{BiSrCaCuO}$ superconducting thin films. J. Appl. Phys., 66(1):457-459, 1989.

[63] Aiko Narazaki, Tadatake Sato, Ryozo Kurosaki, Yoshizo Kawaguchi, and Hiroyuki Niino. Nano- and microdot array formation of $\mathrm{FeSi}_{2}$ by nanosecond excimer laser-induced forward transfer. Applied Physics Express, 1:057001, 2008.

[64] Zhiping Zhou and Wei Ma. Investigation of thermomechanical responses in ultrafast laser heating of metal nanofilms. Thin Solid Films, 519:7940 - 7946, 2011.

[65] Romain Fardel, Matthias Nagel, Frank Nuesch, Thomas Lippert, and Alexander Wokaun. Laser forward transfer using a sacrificial layer: Influence of the material properties. Applied Surface Science, 254:1322 - 1326, 2007.

[66] J. Shaw Stewart, R. Fardel, M. Nagel, P. Delaporte, L. Rapp, C. Cibert, A. p. Alloncle, F. Nüesch, T. Lippert, and A. Wokaun. The effect of laser pulse length upon laser-induced forward transfer using a triazene polymer as a dynamic release layer. Optoelectronics and Advanced Materials, 12:605 - 609, 2010 .

[67] Takashi Mito, Takuji Tsujita, Hiroshi Masuhara, Nobuaki Hayashi, and Kenkichi Suzuki. Hollowing and transfer of polymethyl methacrylate film propelled by laser ablation of triazeno polymer film. Japanese Journal of Applied Physics, 40(8A):L805, 2001.

[68] M. Colina, M. Duocastella, J. M. Fernández-Pradas, P. Serra, and J. L. Morenza. Laser-induced forward transfer of liquids study of the droplet ejection process. Journal of Applied Physics, 99:084909, 2006. 
[69] Koch. Laser printing of skin cells and human stem cells. Tissue Engineering: Part C, 16:847 - 854, 2010.

[70] Béla Hopp, Tomi Smausz, Norbert Kresz, Norbert Barna, Zsolt Bor, Lajos Kolozsvári, Douglas B. Chrisey, András Szabó, and Antal Nógrádi. Survival and proliferative ability of various living cell types after laser-induced forward transfer. Tissue Engineering, 11(11-12):1817 - 1823, 2005.

[71] P. K. Wu, B. R. Ringeisen, J. Callahan, M. Brooks, D. M. Bubb, H. D. Wu, A. Piqué, B. Spargo, R. A. McGill, and D. B. Chrisey. The deposition, structure, pattern deposition, and activity of biomaterial thin-films by matrix-assisted pulsed-laser evaporation (MAPLE) and MAPLE direct write. Thin Solid Films, 398-399(0):607-614, 2001. Proceedings of the 28th International Conference on Metallurgic Coatings and Thin Films.

[72] Z. Kántor, Z. Tóth, T. Szörényi, and A. L. Tóth. Deposition of micrometersized tungsten patterns by laser transfer technique. Applied Physics Letters, 64(25):3506-3508, 1994.

[73] H. Fukumura, Y. Kohji, and H. Masuhara. Laser implantation of fluorescent molecules into polymer films. Applied Surface Science, 9698(0):569 - 571, 1996. Proceedings of Symposium F: Third International Symposium on Laser Ablation of the 1995 E-MRS Spring Conference.

[74] M. Goto, J. Hobley, T. Oishi, A. Kasahara, M. Tosa, K. Yoshihara, M. Kishimoto, and H. Fukumura. Micro-patterning of multiple organic molecules by laser implantation. Applied Physics A, 79(1):157-160, 2004.

[75] N. Seifert, G. Betz, and W. Husinsky. Droplet formation on metallic surfaces during low-fluence laser irradiation. Applied Surface Science, 103:63 - 70, 1996.

[76] N. Seifert, G. Betz, and W. Husinsky. Hydrodynamic phenomena during laser irradiation a finite difference approach. Applied Surface Science, 96:33 - 38, 1996.

[77] N. Seifert and G. Betz. Computer simulations of laser-induced ejection of droplets. Applied Surface Science, 133:189 - 194, 1998.

[78] David A. Willis and Vicentiu Grosu. The effect of melting-induced volumetric expansion on initiation of laser-induced forward transfer. Applied Surface Science, 253:4759 - 4763, 2006.

[79] Dmitriy S. Ivanov, Baerbel Rethfeld, Gerard M. OConnor, Thomas J. Glynn, Alexey N. Volkov, and Leonid V. Zhigilei. The mechanism of nanobump formation in femtosecond pulse laser nanostructuring of thin metal films. Applied Physics A, 92:791 - 796, 2008.

[80] David P. Banks, Kamal S. Kaur, and Robert W. Eason. Influence of optical standing waves on the femtosecond laser-induced forward transfer of transparent thin films. Applied Optics, 48:2058 - 2066, 2009. 
[81] Yuri P. Meshcheryakov, Maxim V. Shugaev, Thomas Mattle, Thomas Lippert, and Nadezhda M. Bulgakova. Role of thermal stresses on pulsed laser irradiation of thin films under conditions of mircobump formation and nonvaporization forward transfer. Applied Physics A, 113:521 - 529, 2013.

[82] Matthew S. Brown, C. Frederik Brasz, Yiannis Ventikos, and Craig B. Arnold. Impulsively actuated jets from thin liquid for high-resolution printing applications. Journal of Fluid Mechanics, 709:341 - 370, 2012.

[83] D. G. Papazoglou, A. Karaiskou, I. Zergioti, and Fotakis. Shadowgraphic imaging of the sub-ps laser-induced forward transfer process. Applied Physics Letters, 81:1594 - 1596, 2002.

[84] Claudia Unger, Jürgen Koch, Ludger Overmeyer, and Boris N. Chichkov. Timeresolved studies of femtosecond-laser induced melt dynamics. Opt. Express, 20(22):24864-24872, Oct 2012.

[85] B. Rethfeld, K. Sokolowski-Tinten, D. von der Linde, and S.I. Anisimov. Timescales in the response of materials to femtosecond laser excitation. Applied Physics A, 79:767 - 769, 2004.

[86] J. Hohlfeld, S.-S. Wellershoff, J. Güdde, U. Conrad, V. Jähnke, and E. Matthias. Electron and lattice dynamics following optical excitation of metals. Chemical Physics, 251:237 - 258, 2000.

[87] Sudhanshu Jha and Chindhu Warke. Interband contributions to optical harmonic generation at a metal surface. Phys. Rev., 153(3):751 - 759, 1967.

[88] Rethfeld. Mikroskopische Prozesse bei der Wechselwirkung von Festkörpern mit Laserpulsen im Subpikosekundenbereich. PhD thesis, Technische Universitaet Carolo-Wilhelmina zu Braunschweig, 2000.

[89] J. K. Chen, J. E. Beraun, and C. L. Tham. Ultrafast thermoelasticity for shortpulse laser heating. International Journal of Engineering Science, 42:793 - 807, 2004 .

[90] Jie Chen, I.V. Tomov, H.E. Elsayed-Ali, and P.M. Rentzepis. Hot electrons blast wave generated by femtosecond laser pulses on thin au(l 111 ) crystal, monitored by subpicosecond x-ray diffraction. Chemical Physics Letters, 419:374 - 378, 2006.

[91] Jing Huang, Yuwen Zhang, and J.K. Chen. Superheating in liquid and solid phases during femtosecond-laser pulse interaction with thin metal film. Applied Physics A, 103(1):113-121, 2011.

[92] B. Rethfeld, K. Sokolowski-Tinten, D. von der Linde, and S. I. Anisimov. Ultrafast thermal melting of laser-excited solids by homogeneous nucleation. Physical Review B, 65:092103, 2002. 
[93] L.M. Cabalin and J.J. Laserna. Experimental determination of laser induced breakdown thresholds of metals under nanosecond q-switched laser operation. Spectrochimica Acta Part B, 53:723 - 730, 1998.

[94] M.I. Kaganov and L.V. Tanatarov I.M. Lifshitz. Relaxation between electrons and the crystalline lattice. Sov. Phys., 173, 1957.

[95] Sergei I. Anisimov and Baerbel Rethfeld. Theory of ultrashort laser pulse interaction with a metal. In Proc. SPIE 3093, Nonresonant Laser-Matter Interaction (NLMI-9), volume 192, 1997.

[96] J. K. Chen, J. E. Beraun, and C. L. Tham. Investigation of thermal response caused by pulse laser heating. Numerical Heat Transfer, Part A: Applications: An International Journal of Computation and Methodology, 44:705 - 722, 2003.

[97] S.-S.Wellershoff, J. Hohlfeld, J. Güdde, and E. Matthias. The role of electronphonon coupling in femtosecond laser damage of metals. Applied Physics A, 69:99 - 107, 1999.

[98] J. M. Liu. Simple technique for measurements of pulsed gaussian-beam spot sizes. Optics Letters, 7:196-198, 1982.

[99] Menzel-Gläser. www.menzel.de, January 2015.

[100] R. Pohl, C. W. Visser, G. R. B. E. Römer, C. Sun, A. J. Huis in 't Veld, and D. Lohse. High-resolution imaging of ejection dynamics in laserinduced forward transfer. In Yoshiki Nakata, Xianfan Xu, Stephan Roth, and Beat Neuenschwander, editors, Laser Applications in Microelectronic and Optoelectronic Manufacturing, page 89670X, March 2014.

[101] Jozsef Garai. Physical model for vaporization. Fluid Phase Equilibria, 283:89 92, 2009.

[102] X. Zeng, X.L. Mao, R. Greif, and R.E. Russo. Experimental investigation of ablation efficiency and plasma expansion during femtosecond and nanosecond laser ablation of silicon. Applied Physics A, 80(2):237-241, July 2004.

[103] Ralph Pohl, CW Visser, G Römer, Chao Sun, and Detlef Lohse. Droplet ejection in laser-induced forward transfer: mechanism for droplet fragmentation. In Proceedings of LAMP, 2013.

[104] F. Korte, J. Koch, and B.N. Chichkov. Formation of microbumps and nanojets on gold targets by femtosecond laser pulses. Applied Physics A, 79(4-6):879-881, July 2004.

[105] A. I. Kuznetsov, J. Koch, and B. N. Chichkov. Nanostructuring of thin gold films by femtosecond lasers. Applied Physics A, 94(2):221-230, August 2009.

[106] Romain Fardel. Laser-Induced Forward Transfer for the Fabrication of Patterned Organic Light-Emitting Diodes. PhD thesis, ETH Zurich, 2009. 
[107] B. N. Chichkov, C. Momma, S. Nolte, F. von Alvensleben, and A. Tunnermann. Femtosecond, picosecond and nanosecond laser ablation of solids. Applied Physics A, 63:109-115, 1996.

[108] Vicentiu Grosu David A. Willis. Evaporation and phase explosion during laserinduced forward transfer of aluminum. In Porceedings of SPIE, 2004.

[109] H. Lhuisser and E. Villermaux. Bursting bubble aerosols. Journal of Fluid Mechanics, 696:5-44, 2012.

[110] Gareth H. Mckinley. Visco-elasto-capillary thinning and break-up of complex fluids. The British Society of Rheology, pages 1-49, 2005.

[111] D.A. Harrison, D. Yan, and S. Blairs. The surface tension of liquid copper. The Journal of Chemical Thermodynamics, 9(12):1111 - 1119, 1977.

[112] Caroline Desgranges and Jerome Delhommelle. Shear viscosity of liquid copper at experimentally accessible shear rates: Application of the transient-time correlation function formalism. The Journal of Chemical Physics, 128(8):-, 2008 .

[113] Yoshiyuki Tagawa, Nikolai Oudalov, Claas Willem Visser, Ivo R. Peters, Devaraj van der Meer, Chao Sun, Andrea Prosperetti, and Detlef Lohse. Highly focused supersonic microjets. Phys. Rev. X, 2(3):031002, July 2012.

[114] Romain Fardel, Matthias Nagel, Frank Nuesch, Thomas Lippert, and Alexander Wokaun. Shadowgraphy investigation of laser-induced forward transfer: Front side and back side ablation of the triazene polymer sacrificial layer. Applied Surface Science, 255:5430 - 5434, 2009.

[115] Xianfan Xu and David A. Willis. Non-equilibrium phase change in metal induced by nanosecond pulsed laser irradiation. Journal of Heat Transfer, 2002.

[116] Chengping $\mathrm{Wu}$ and Leonid V. Zhigilei. Microscopic mechanisms of laser spallation and ablation of metal targets from large-scale molecular dynamics simulations. Applied Physics A, 2014.

[117] Adela Ben-Yakar Samuel X Guo. Femtosecond laser nanoablation of glass in the near-field of single wall carbon nanotube bundles. Applied Physics, 41:185306, 2008 .

[118] Mohammad Vaezi, Hermann Seitz, and Shoufeng Yang. A review on 3D microadditive manufacturing technologies. The International Journal of Advanced Manufacturing Technology, 67(5-8):1721-1754, November 2013.

[119] Wenbin Cao and Yoshinari Miyamoto. Freeform fabrication of aluminum parts by direct deposition of molten aluminum. Journal of Materials Processing Technology, 173(2):209-212, April 2006. 
[120] Jiwen Wang, Raymond C Y Auyeung, Heungsoo Kim, Nicholas a Charipar, and Alberto Piqué. Three-dimensional printing of interconnects by laser direct-write of silver nanopastes. Advanced Materials, 22(40):4462-6, October 2010.

[121] Song-yi Zhong, Le-hua Qi, Jun Luo, Han-song Zuo, Xiang-hui Hou, and Hejun Li. Effect of process parameters on copper droplet ejecting by pneumatic drop-on-demand technology. Journal of Materials Processing Technology, 214(12):3089-3097, 2014.

[122] Yuang-Cherng Chiou, Rong-Tsong Lee, Tai-Jia Chen, and Jing-Mei Chiou. Fabrication of high aspect ratio micro-rod using a novel electrochemical micromachining method. Precision Engineering, 36(2):193-202, April 2012.

[123] Ralph Pohl, Claas Willem Visser, Gert-Willem Römer, Detlef Lohse, Chao Sun, and Bert Huis in t Veld. Ejection Regimes in Picosecond Laser-Induced Forward Transfer of Metals. Physical Review Applied, 3(2):024001, 2015.

[124] Claas Willem Visser, Philipp Erhard Frommhold, Sander Wildeman, Robert Mettin, Detlef Lohse, and Chao Sun. Dynamics of high-speed micro-drop impact: numerical simulations and experiments at frame-to-frame times below 100 ns. Soft Matter, 11:1708-1722, December 2015.

[125] D Zrnic and DS Swatik. On the resistivity and surface tension of the eutectic alloy of gallium and indium. Journal of the less common metals, 18:67-68, 1969.

[126] Ching-Yue Wang Li Yang. Experimental study on microdeposition of the copper thin film by femtosecond laser-induced forward transfer. Chinese Physics B, 18:4292, 2009.

[127] A. Palla-Papavlu, V Dinca, C Luculescu, J Shaw-Stewart, M Nagel, T Lippert, and M Dinescu. Laser induced forward transfer of soft materials. Journal of Optics, 12(12):124014, December 2010.

[128] A. Klini, P. A. Loukakos, D. Gray, A. Manousaki, and C. Fotakis. Laser induced forward transfer of metals by temporally shaped femtosecond laser pulses. Optics Express, 16:11300, 2008.

[129] Dirkjan B. van Dam and Christophe Le Clerc. Experimental study of the impact of an ink-jet printed droplet on a solid substrate. Phys. Fluids, 16:3403-3414, 2004 .

[130] A. McDonald, M. Lamontagne, C. Moreau, and S. Chandra. Impact of plasmasprayed metal particles on hot and cold glass surfaces. Thin Solid Films, 514(12):212-222, August 2006.

[131] David Paul Banks. Femtosecond Laser Induced Forward Transfer Techniques for the Deposition of Nanoscale, Intact, and Solid Phase Material. PhD thesis, University of Southampton, 2008. 
[132] Raymond C. Y. Auyeung, Heungsoo Kim, Scott Mathews, and Alberto Piqué. Laser forward transfer using structured light. Opt. Express, 23(1):422-430, Jan 2015.

[133] Ludovic Rapp, Catalin Constantinescu, Yannick Larmande, AnnePatricia Alloncle, and Philippe Delaporte. Smart beam shaping for the deposition of solid polymeric material by laser forward transfer. Applied Physics A, 117(1):333$339,2014$.

[134] Jeffrey A. Davis, Dylan E. McNamara, Don M. Cottrell, and Tomio Sonehara. Two-dimensional polarization encoding with a phase-only liquid-crystal spatial light modulator. Appl. Opt., 39(10):1549-1554, 2000.

[135] Dun Liu, Walter Perrie, Leigh Mellor, Zheng Kuang, Eamonn Fearon, Stuart Edwardson, Geoff Dearden, and Ken Watkins. Picosecond laser beam shaping using a spatial light modulator. In ICALEO, pages 735-738. ICALEO, 2012.

[136] Hamamatsu Photonics. LCOS-SLM (Liquid Crystal on Silicon - Spatial Light Modulator), January 2014. KACC1172E11.

[137] Jinyang Liang, Rudolph N. Kohn Jr., Michael F. Becker, Daniel J. Heinzen, et al. High-precision laser beam shaping using a binary-amplitude spatial light modulator. Appl. Opt., 49(8):1323-1330, 2010.

[138] Dieter Bäuerle. Laser Processing and Chemistry, volume 3. Springer, 2000.

[139] Aleksandar D. Rakic, Aleksandra B. Djurišic, Jovan M. Elazar, and Marian L. Majewski. Optical properties of metallic films for vertical-cavity optoelectronic devices. Appl. Opt., 37(22):5271-5283, 1998. 


\section{Acknowledgments}

I would like to express my gratitude to all the people that have supported me during the past four years.

First, I acknowledge Just Herder for his kind support and for accepting my thesis as a promoter. I thank my daily supervisor Gert-Willem Römer for the countless hours he spent on my behalf and for being the person who he is. I am also grateful to Martina Tjapkes for the constant supply of coffee and for keeping up the good mood in the group. I acknowledge the members of the graduation committee prof. Philippe Delaporte, prof. Quan Zhou, prof. Detlef Lohse, prof. Remko Akkerman and dr. Ton Bor for evaluating my thesis. I am thankful to Bert Huis in 't Veld for accepting me as a $\mathrm{PhD}$ in the group of ALT and for initiating my research in this group.

Moreover, the work that I have performed would not have been possible without the support of certain people. I am grateful to Gerald Ebberink for several discussions on my research and for the countless hours he has spent in the clean room on my behalf. I thank Leo Tiemersma for the fabrication of the mechanical parts of my LIFT setup. I am grateful to Laura Vargas, Henk van Wolferen and Mark Smithers for supporting me with the SEM and FIB analysis tools. I acknowledge Marc Hoppenbrouwers and An Prenen for the joint experiments in the initial stage of my $\mathrm{PhD}$. I am grateful for the intense cooperation with Claas-Willem Visser and the countless discussions and coffees we have had during the past years. Special thanks to my master students Mark, Mart and Luigi for their great work and for accepting me as their daily supervisor. I would also like to thank the staff of the Mechanical Automation Group: Ronald, Dannis, Johannes, Pathiraj, Jaap, Wouter and Jonathan as well as our colleagues at LAC, Kite Robotics and Lightmotif. I thank my $\mathrm{PhD}$ colleagues who made my stay in this group special: Paul, Johan, Jozef, Ali, Justus, Steven, Volkert, Jan, Ger and Bert. A special thanks goes to my closest colleagues and friends Niya, Davide and Daniel. It was a great pleasure to be part of your group.

Finally, I would like to thank my parents Magdalene and Werner, my brother 
Christian and my beloved girlfriend Gerlind for their patience and their generous support during these challenging, but exciting times. 